\title{
The Auslander bijections: how morphisms are determined by modules
}

\author{
Claus Michael Ringel
}

Received: 7 January 2013 / Revised: 3 June 2013 / Accepted: 4 June 2013 / Published online: 3 July 2013 (C) The Author(s) 2013. This article is published with open access at SpringerLink.com

\begin{abstract}
Let $\Lambda$ be an artin algebra. In his seminal Philadelphia Notes published in 1978, Auslander introduced the concept of morphisms being determined by modules. Auslander was very passionate about these investigations (they also form part of the final chapter of the Auslander-Reiten-Smalø book and could and should be seen as its culmination). The theory presented by Auslander has to be considered as an exciting frame for working with the category of $\Lambda$-modules, incorporating all what is known about irreducible maps (the usual Auslander-Reiten theory), but the frame is much wider and allows for example to take into account families of modulesan important feature of module categories. What Auslander has achieved is a clear description of the poset structure of the category of $\Lambda$-modules as well as a blueprint for interrelating individual modules and families of modules. Auslander has subsumed his considerations under the heading of "morphisms being determined by modules". Unfortunately, the wording in itself seems to be somewhat misleading, and the basic definition may look quite technical and unattractive, at least at first sight. This could be the reason that for over 30 years, Auslander's powerful results did not gain the attention they deserve. The aim of this survey is to outline the general setting for Auslander's ideas and to show the wealth of these ideas by exhibiting many examples.
\end{abstract}

Communicated by S.K. Jain.

C. M. Ringel ( $\square)$

Department of Mathematics, Shanghai Jiao Tong University,

Shanghai 200240, People's Republic of China

e-mail: ringel@math.uni-bielefeld.de

C. M. Ringel

King Abdulaziz University, P O Box 80200, Jeddah, Saudi Arabia 
Keywords Auslander bijections · Auslander-Reiten theory · Right factorization lattice - Morphisms determined by modules - Finite length categories: global directedness - Local symmetries · Representation type · Brauer-Thrall conjectures · Riedtmann-Zwara degenerations · Hammocks · Kronecker quiver · Quiver Grassmannians · Auslander varieties · Modular lattices · Meet semi-lattices

Mathematics Subject Classification (2010) Primary 16G70 - 18E10 - 18A25 . 18A32 - 16G60; Secondary 16G20 · 18A20 - 06C05 · 14M15 · 19A49 · 03C60

\section{Introduction}

There are two basic mathematical structures: groups and lattices, or, more generally, semigroups and posets. A first glance at any category should focus the attention on these two structures: to symmetry groups (for example the automorphism groups of the individual objects), as well as to the posets given by suitable sets of morphisms, for example by looking at inclusion maps (thus dealing with the poset of all subobjects of an object), or at the possible factorizations of morphisms. In this way, one distinguishes between local symmetries and global directedness.

The present survey deals with the category mod $\Lambda$ of finite length modules over an artin algebra $\Lambda$. Its aim is to report on the work of Auslander in his seminal Philadelphia Notes published in 1978. Auslander was very passionate about these investigations and they also form part of the final chapter of the Auslander-Reiten-Smalø book: there, they could (and should) be seen as a kind of culmination. It seems to be surprising that the feedback until now is quite meager. After all, the theory presented by Auslander has to be considered as an exciting frame for working with the category $\bmod \Lambda$, incorporating what is called the Auslander-Reiten theory (to deal with the irreducible maps), but this frame is much wider and allows for example to take into account families of modules - an important feature of a module category. Indeed, many of the concepts which are relevant when considering the categories $\bmod \Lambda$ fit into the frame! What Auslander has achieved (but he himself may not have realized it) was a clear description of the poset structure of $\bmod \Lambda$ and of the interplay between families of modules.

Auslander's considerations are subsumed under the heading of morphisms being determined by modules, but the wording in itself seems to be somewhat misleading, and the basic definition looks quite technical and unattractive, at least at first sight. This could be the reason that for over 30 years, Auslander's powerful results did not gain the attention they deserve.

Here is a short summary: Let $\Lambda$ be an artin algebra. The modules which we consider will be left $\Lambda$-modules of finite length, and maps (or morphisms) will be $\Lambda$-module homomorphisms, unless otherwise specified. Auslander asks for a description of the class of maps ending in a fixed module $Y$. Two maps $f: X \rightarrow Y$ and $f^{\prime}: X^{\prime} \rightarrow Y$ are said to be right equivalent provided there are maps $h: X \rightarrow X^{\prime}$ and $h^{\prime}: X^{\prime} \rightarrow X$ such that $f=f^{\prime} h$ and $f^{\prime}=f h^{\prime}$. The right equivalence class of $f$ will be denoted by $[f\rangle$. The object studied by Auslander is the set of right equivalence classes of maps 
ending in $Y$, we denote this set by

$$
[\rightarrow Y\rangle
$$

It is a poset via the relation $\leq$ which is defined as follows:

$$
[f: X \rightarrow Y\rangle \leq\left[f^{\prime}: X^{\prime} \rightarrow Y\right\rangle
$$

provided there is a homomorphism $h: X \rightarrow X^{\prime}$ with $f=f^{\prime} h$, thus provided the following diagram commutes:

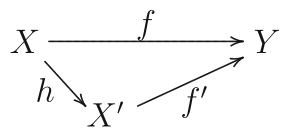

It is easy to see that the poset $[\rightarrow Y\rangle$ is a lattice, thus we call it the right factorization lattice for $Y$.

Looking at maps $f: X \rightarrow Y$, we may (and often will) assume that $f$ is right minimal, thus that there is no non-zero direct summand $X^{\prime}$ of $X$ with $f\left(X^{\prime}\right)=0$. Note that any right equivalence class contains a right minimal map, and if $f: X \rightarrow Y$ and $f^{\prime}: X^{\prime} \rightarrow Y$ are right minimal maps, then any $h: X \rightarrow X^{\prime}$ with $f=f^{\prime} h$ has to be an isomorphism.

Of course, to analyze the poset $[\rightarrow Y\rangle$ is strongly related to a study of the contravariant Hom-functor $\operatorname{Hom}(-, Y)$, however the different nature of these two mathematical structures should be stressed: $\operatorname{Hom}(-, Y)$ is an additive functor whereas $[\rightarrow Y\rangle$ is a poset, and it is the collection of these posets $[\rightarrow Y\rangle$ which demonstrates the global directedness.

In general, the right factorization lattice $[\rightarrow Y\rangle$ is very large and does not satisfy any chain condition. The main idea of Auslander is to write $[\rightarrow Y\rangle$ as the filtered union of the subsets ${ }^{C}[\rightarrow Y\rangle$, where ${ }^{C}[\rightarrow Y\rangle$ is given by those maps $f$ which are "right $C$-determined". These posets are again lattices and they are of finite height, we call ${ }^{C}[\rightarrow Y\rangle$ the right $C$-factorization lattice for $Y$. Since the concept of "right determination" looks (at least at first sight) technical and unattractive, let us first describe the set ${ }^{C}[\rightarrow Y\rangle$ only in the important case when $C$ is a generator: in this case, ${ }^{C}[\rightarrow Y\rangle$ consists of the (right equivalence classes of the right minimal) maps $f$ ending in $Y$ with kernel in add $\tau C$ (we denote by $\tau=D \operatorname{Tr}$ and $\tau^{-}=\operatorname{Tr} D$ the Auslander-Reiten translations). Here is Auslander's first main assertion:

$$
[\rightarrow Y\rangle=\bigcup_{C}{ }^{C}[\rightarrow Y\rangle
$$

where $C$ runs through all isomorphism classes of $\Lambda$-modules; or, in the formulation of Auslander: any map in mod $\Lambda$ is right determined by some module $C$. Note that the inclusion of the lattice ${ }^{C}[\rightarrow Y\rangle$ into the lattice $[\rightarrow Y\rangle$ preserves meets, but usually not joins. 
Auslander's second main assertion describes the right $C$-factorization lattice $C_{[\rightarrow Y\rangle}$ as follows: There is a lattice isomorphism

$$
\eta_{C Y}:{ }^{C}[\rightarrow Y\rangle \longrightarrow \mathcal{S} \operatorname{Hom}(C, Y)
$$

where $\operatorname{Hom}(C, Y)$ is considered as an $\operatorname{End}(C)^{\mathrm{op}}$-module, and where $\mathcal{S} M$ denotes the submodule lattice of a module $M$. Actually, the map $\eta_{C Y}$ is easy to describe, namely $\eta_{C Y}(f)=\operatorname{Im} \operatorname{Hom}(C, f)$ for $f$ a morphism ending in $Y$. The essential assertion is the surjectivity of $\eta_{C Y}$, thus to say that any submodule of $\operatorname{Hom}(C, Y)$ is of the form $\operatorname{Im} \operatorname{Hom}(C, f)$ for some $f$.

What is the relevance? As we have mentioned, usually the lattice $[\rightarrow Y\rangle$ itself will not satisfy any chain conditions, but all the lattices ${ }^{C}[\rightarrow Y\rangle$ are of finite height and often can be displayed very nicely: according to (2) we deal with the submodule lattice $\mathcal{S} M$ of some finite length module $M$ over an artin algebra (namely over $\Gamma(C)=\operatorname{End}(C)^{\text {op }}$ ) and it is easy to see that any submodule lattice arises in this way. Using the Auslander bijections $\eta_{C Y}$, one may transfer properties of submodule lattices to the right $C$ factorization lattices ${ }^{C}[\rightarrow Y\rangle$, this will be one of the aims of this paper. Given a submodule $U$ of $\operatorname{Hom}(C, Y)$, let $f$ be a right $C$-determined map ending in $Y$ such that $\eta_{C Y}(f)=U$. The composition series of the factor module $\operatorname{Hom}(C, Y) / U$ correspond to certain factorizations of $f$ (to the "maximal $C$-factorizations"), and we may define the $C$-type of $f$ so that it is equal to the dimension vector of the module $\operatorname{Hom}(C, Y) / U$ (recall that the dimension vector of a module $M$ has as coefficients the Jordan-Hölder multiplicities of the various simple modules occurring in $M$ ).

Submodule lattices have interesting combinatorial features, and it seems to interesting that Auslander himself looked mainly at combinatorial properties (for example at waists in submodule lattices). But we should stress that we really are in the realm of algebraic geometry. Thus, let us assume for a moment that $\Lambda$ is a $k$-algebra where $k$ is an algebraically closed field. If $M$ is a finite-dimensional $\Lambda$-module, the set $\mathcal{S} M$ of all submodules of $M$ is the disjoint union of the sets $\mathbb{G}_{\mathbf{e}}(M)$ consisting of all submodules of $M$ with fixed dimension vector e. It is well-known that $\mathbb{G}_{\mathbf{e}}(M)$ is in a natural way a projective variety, called nowadays a quiver Grassmannian. Given $\Lambda$-modules $C$ and $Y$, the Auslander bijections draw the attention on the $\operatorname{End}(C)^{\text {op }}$ module $M=\operatorname{Hom}(C, Y)$, let $\mathbf{d}$ be its dimension vector and let $\mathbf{e}, \mathbf{e}^{\prime}$ be dimension vectors with $\mathbf{e}+\mathbf{e}^{\prime}=\mathbf{d}$. The quiver Grassmannians $\mathbb{G}_{\mathbf{e}^{\prime}}(\operatorname{Hom}(C, Y))$ corresponds under the Auslander bijection $\eta_{C Y}$ to the set ${ }^{C}[\rightarrow Y\rangle^{\mathbf{e}}$ of all right equivalence classes of right $C$-determined maps which end in $Y$ and have type e. We call ${ }^{C}[\rightarrow Y\rangle^{\mathbf{e}}$ an Auslander variety. These Auslander varieties have to be considered as an important tool for studying the right equivalence classes of maps ending in a given module.

We end this summary by an outline in which way the Auslander bijections (2) incorporate the existence of minimal right almost split maps: we have to look at the special case where $Y$ is indecomposable and $C=Y$ and to deal with the submodule $\operatorname{rad}(Y, Y)$ of $\operatorname{Hom}(Y, Y)$. The bijection (2) yields an element $f: X \rightarrow Y$ in ${ }^{Y}[\rightarrow Y\rangle$ such that $\eta_{Y Y}(f)=\operatorname{rad}(Y, Y)$; to say that $f$ is right $Y$-determined means that $f$ is right almost split.

The survey is divided into three parts. Part I presents the general setting, it comprises the Sects. 2 to 10 . The Sects. 11 to 15 form Part II, here we show in which way the 
Auslander bijections deal with families of modules. Finally, in Parts III, we discuss some special cases; these are the Sects. 16 to 18.

\section{The setting}

\section{The right factorization lattice $[\rightarrow Y\rangle$}

Let $Y$ be a $\Lambda$-module. Let $\bigsqcup_{X} \operatorname{Hom}(X, Y)$ be the class of all homomorphisms $f: X \rightarrow Y$ with arbitrary modules $X$ (such homomorphisms will be said to be the homomorphisms ending in $Y$ ). We define a preorder $\preceq$ on this class as follows: Given $f: X \rightarrow Y$ and $f^{\prime}: X^{\prime} \rightarrow Y$, we write $f \preceq f^{\prime}$ provided there is a homomorphism $h: X \rightarrow X^{\prime}$ such that $f=f^{\prime} h$ (clearly, this relation is reflexive and transitive). As usual, such a preorder defines an equivalence relation (in our setting, we call it right equivalence) by saying that $f, f^{\prime}$ are right equivalent provided we have both $f \preceq f^{\prime}$ and $f^{\prime} \preceq f$, and it induces a poset relation $\leq$ on the set $[\rightarrow Y\rangle$ of right equivalence classes of homomorphisms ending in $Y$. Given a morphism $f: X \rightarrow Y$, we denote its right equivalence class by $[f\rangle$ and by definition $[f\rangle \leq\left[f^{\prime}\right\rangle$ if and only if $f \preceq f^{\prime}$. As we will see in Proposition 2.2, the poset $[\rightarrow Y\rangle$ is a lattice, thus we will call it the right factorization lattice for $Y$.

It should be stressed that $[\rightarrow Y\rangle$ is a set, not only a class: namely, the isomorphism classes of $\Lambda$-modules form a set and for every module $X$, the homomorphisms $X \rightarrow Y$ form a set; we may choose a representative from each isomorphism class of $\Lambda$-modules and given a homomorphism $f: X \rightarrow Y$, then there is an isomorphism $h: X^{\prime} \rightarrow X$ where $X^{\prime}$ is such a representative, and $f$ is right equivalent to $f h$.

Recall that a map $f: X \rightarrow Y$ is said to be right minimal provided any direct summand $X^{\prime}$ of $X$ with $f\left(X^{\prime}\right)=0$ is equal to zero. If $f: X \rightarrow Y$ is a morphism and $X=X^{\prime} \oplus X^{\prime \prime}$ such that $f\left(X^{\prime \prime}\right)=0$ and $f \mid X^{\prime}: X^{\prime} \rightarrow Y$ is right minimal, then $f \mid X^{\prime}$ is called a right minimalisation of $f$. The kernel of a right minimalisation of $f$ will be called the intrinsic kernel off, it is unique up to isomorphism.

Proposition 2.1 Every right equivalence class $[f\rangle$ in $[\rightarrow Y\rangle$ contains a right minimal morphism, namely $\left[f^{\prime}\right\rangle$, where $f^{\prime}$ is a right minimalisation of $f$. Given right minimal morphisms $f: X \rightarrow Y$ and $f^{\prime}: X^{\prime} \rightarrow Y$, then $f, f^{\prime}$ are right equivalent if and only if there is an isomorphism $h: X \rightarrow X^{\prime}$ such that $f=f^{\prime} h$.

Proof Let $f: X \rightarrow Y$ be a homomorphism ending in $Y$. Write $X=X_{1} \oplus X_{2}$ such that $f\left(X_{2}\right)=0$ and $f \mid X_{1}: X_{1} \rightarrow Y$ is right minimal. Let $u: X_{1} \rightarrow X_{1} \oplus X_{2}$ be the canonical inclusion, $p: X_{1} \oplus X_{2} \rightarrow X_{1}$ the canonical projection. Then $p u=1_{X_{1}}$ and $f=f u p$ (since $f\left(X_{2}\right)=0$ ). We see that $f u \preceq f$ and $f=f u p \preceq f u$, thus $f$ and $f u$ are right equivalent and $f u=f \mid X_{1}$ is right minimal. If the right minimal morphisms $f: X \rightarrow Y$ and $f^{\prime}: X^{\prime} \rightarrow Y$ are right equivalent, then there are morphisms $h: X \rightarrow X^{\prime}$ and $h^{\prime}: X^{\prime} \rightarrow Y$ such that $f=f^{\prime} h$ and $f^{\prime}=f h^{\prime}$. But $f=f h^{\prime} h$ implies that $h^{\prime} h$ is an automorphism, and $f^{\prime}=f^{\prime} h h^{\prime}$ implies that $h h^{\prime}$ is an automorphism, thus $h, h^{\prime}$ have to be isomorphisms (see [5] I.2).

Remark Monomorphisms $X \rightarrow Y$ are always right minimal, and the right equivalence classes of monomorphisms ending in $Y$ may be identified with the submodules of $Y$ 
(here, we identify the right equivalence class of the monomorphism $f: X \rightarrow Y$ with the image of $X$ ).

Proposition 2.2 The poset $[\rightarrow Y\rangle$ is a lattice with zero and one. Given $f_{1}: X_{1} \rightarrow Y$ and $f_{2}: X_{2} \rightarrow Y$, say with pullback $g_{1}: X \rightarrow X_{1}, g_{2}: X \rightarrow X_{2}$, the meet of $\left[f_{1}\right\rangle$ and $\left[f_{2}\right\rangle$ is given by the map $f_{1} g_{1}: X \rightarrow Y$, the join of $\left[f_{1}\right\rangle$ and $\left[f_{2}\right\rangle$ is given by $\left[f_{1}, f_{2}\right]: X_{1} \oplus X_{2} \rightarrow Y$.

Proof (a trivial verification) Write $f=f_{1} g_{1}=f_{2} g_{2}$. We have $f=f_{1} g_{1} \preceq f_{1}$ and $f=f_{2} g_{2} \preceq f_{2}$, thus $[f\rangle \leq\left[f_{1}\right\rangle$ and $[f\rangle \leq\left[f_{2}\right\rangle$. If $f^{\prime}: X^{\prime} \rightarrow Y$ is a morphism with $\left[f^{\prime}\right\rangle \leq\left[f_{1}\right\rangle$ and $\left[f^{\prime}\right\rangle \leq\left[f_{2}\right\rangle$, then $f^{\prime} \preceq f_{1}$ and $f^{\prime} \preceq f_{2}$, thus there are morphisms $\phi_{i}$ with $f^{\prime}=f_{i} \phi_{i}$, for $i=1$, 2 Since $f_{1} \phi_{1}=f_{2} \phi_{2}$, the pullback property yields a morphism $\phi: X^{\prime} \rightarrow X$ such that $\phi_{i}=g_{i} \phi$ for $i=1,2$. Thus $f^{\prime}=f_{1} \phi_{1}=f_{1} g_{1} \phi=f \phi$ shows that $f^{\prime} \preceq f$, thus $\left[f^{\prime}\right\rangle \leq[f\rangle$. This shows that $[f\rangle$ is the meet of $\left[f_{1}\right\rangle$ and $\left[f_{2}\right\rangle$.

Second, denote the canonical inclusion maps $X_{i} \rightarrow X_{1} \oplus X_{2}$ by $u_{i}$, for $i=1,2$, thus $\left[f_{1}, f_{2}\right] u_{i}=f_{i}$ and therefore $\left[f_{i}\right\rangle \leq\left[\left[f_{1}, f_{2}\right]\right\rangle$ for $i=1,2$. Assume that there is given a morphism $g: X^{\prime \prime} \rightarrow Y$ with $\left[f_{i}\right\rangle \leq[g\rangle$ for $i=1,2$. This means that there are morphisms $\psi_{i}: X_{i} \rightarrow X^{\prime \prime}$ such that $f_{i}=g \psi_{i}$ for $i=1,2$. Let $\psi=\left[\psi_{1}, \psi_{2}\right]: X_{1} \oplus X_{2} \rightarrow X^{\prime \prime}$ (with $\psi u_{i}=\psi_{i}$ ). Then $\left[f_{1}, f_{2}\right]=g\left[\psi_{1}, \psi_{2}\right]=g \psi$ shows that $\left[f_{1}, f_{2}\right] \preceq g$, thus $\left[\left[f_{1}, f_{2}\right]\right\rangle \leq[g\rangle$. This shows that $\left[\left[f_{1}, f_{2}\right]\right\rangle$ is the join of $\left[f_{1}\right\rangle$ and $\left[f_{2}\right\rangle$.

It is easy to check that the map $0 \rightarrow Y$ is the zero element of $[\rightarrow Y\rangle$ and that the identity map $Y \rightarrow Y$ is its unit element.

It should be stressed that if $f_{1}: X_{1} \rightarrow Y$ and $f_{2}: X_{2} \rightarrow Y$ are right minimal, say with pullback $g_{1}: X \rightarrow X_{1}, g_{2}: X \rightarrow X_{2}$, then neither the map $f_{1} g_{1}$ nor the direct sum map $\left[f_{1}, f_{2}\right]: X_{1} \oplus X_{2} \rightarrow Y$ will be right minimal, in general. Thus if one wants to work with right minimal maps, one has to right minimalise the maps in question. Here are corresponding examples:

Example 1 Let $\Lambda$ be the path algebra of the quiver

$$
a \longleftarrow b
$$

of type $\mathbb{A}_{2}$.

All path algebras of quivers considered in the paper will have coefficients in an arbitrary field $k$, unless we specify some further conditions. When dealing with the path algebra of a quiver $\Delta$, and $x$ is a vertex of $\Delta$, we denote by $S(x)$ (or also just by $x$ ) the simple module corresponding to $x$, by $P(x)$ and $Q(x)$ the projective cover or injective envelope of $S(x)$, respectively.

Take as maps $f_{1}, f_{2}$ the canonical projection $f_{1}=f_{2}: P(b) \rightarrow S(b)$, this is a right minimal map. The pullback $U$ of $f_{1}$ and $f_{2}$ is a submodule of $P(b) \oplus P(b)$ which is isomorphic to $S(a) \oplus P(b)$. Since any map $S(a) \rightarrow S(b)$ is zero, there is no right minimal map $U \rightarrow S(b)$.

Also, the map $\left[f_{1}, f_{2}\right]: P(b) \oplus P(b) \rightarrow S(b)$ is not right minimal, since we have $\operatorname{dim} \operatorname{Hom}(P(b), S(b))=1$. 
As we have seen, the poset $[\rightarrow Y\rangle$ is a lattice. What will be important in the following discussion is the fact that we deal with a meet-semilattice (these are the posets such that any pair of elements has a meet). Note that all the semilattices which we deal with turn out to be lattices, however the poset maps to be considered will preserve meets, but usually not joins, thus we really work in the category of meetsemilattices.

Proposition 2.3 The lattice $[\rightarrow Y\rangle$ is modular.

Proof Let $f_{i}: X_{i} \rightarrow Y$ be maps with target $Y$, where $1 \leq i \leq 3$, such that $f_{1} \preceq f_{3}$. We want to show that

$$
\left.\left(\left[f_{1}\right\rangle \vee\left[f_{2}\right\rangle\right) \wedge\left[f_{3}\right\rangle \leq\left[f_{1}\right\rangle \vee\left(\left[f_{2}\right\rangle\right) \wedge\left[f_{3}\right\rangle\right)
$$

the reverse inequality being trivial. Since $f_{1} \preceq f_{3}$, there is $h: X_{1} \rightarrow X_{3}$ such that $f_{1}=f_{3} h$.

First, let us construct an element $f$ in $\left.\left[f_{1}\right\rangle \vee\left(\left[f_{2}\right\rangle\right) \wedge\left[f_{3}\right\rangle\right)$. A pullback diagram

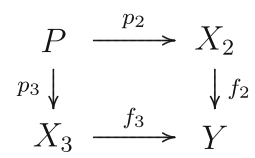

yields a map $f_{3} p_{3}$ such that $\left.\left[f_{3} p_{3}\right\rangle=\left[f_{2}\right\rangle\right) \wedge\left[f_{3}\right\rangle$, thus the map $f=\left[f_{3} h, f_{3} p_{3}\right]$ : $X_{1} \oplus P \rightarrow Y$ belongs to $\left.\left[f_{3} h\right\rangle \vee\left(\left[f_{2}\right\rangle\right) \wedge\left[f_{3}\right\rangle\right)$.

Next, we construct an element $f^{\prime}$ in $\left(\left[f_{1}\right\rangle \vee\left[f_{2}\right\rangle\right) \wedge\left[f_{3}\right\rangle$. The map $\left[f_{3} h, f_{2}\right]$ : $X_{1} \oplus X_{2} \rightarrow Y$ belongs to $\left[f_{1}\right\rangle \vee\left[f_{2}\right\rangle$, now we form the pullback

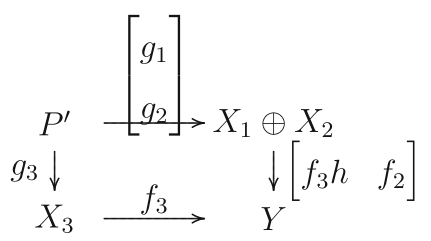

the map $f^{\prime}=f_{3} g_{3}=\left[f_{3} h, f_{2}\right]\left[\begin{array}{l}g_{1} \\ g_{2}\end{array}\right]$ is an element of $\left(\left[f_{3} h\right\rangle \vee\left[f_{2}\right\rangle\right) \wedge\left[f_{3}\right\rangle$.

It follows from $f_{3} g_{3}=\left[f_{3} h, f_{2}\right]\left[\begin{array}{l}g_{1} \\ g_{2}\end{array}\right]=f_{3} h g_{1}+f_{2} g_{2}$ that $f_{2} g_{2}=f_{3}\left(g_{3}-h g_{1}\right)$, thus the pair of maps $g_{2}, g_{3}-h g_{1}$ factors through the pullback $P$ of $f_{2}, f_{3}$. Thus there is $g: P^{\prime} \rightarrow P$ such that $p_{2} g=g_{2}$ and $p_{3} g=g_{3}-h g_{1}$. It follows that $g_{3}=h g_{1}+p_{3} g$ and therefore

$$
f=f_{3} g_{3}=f_{3} h g_{1}+f_{3} p_{3} g=\left[f_{3} h, f_{3} p_{3}\right]\left[\begin{array}{c}
g_{1} \\
g
\end{array}\right]=f^{\prime}\left[\begin{array}{c}
g_{1} \\
g
\end{array}\right] .
$$

This shows that $f \preceq f^{\prime}$.

Example 2 Failure of the chain conditions. Here are examples which show that in general $[\rightarrow Y\rangle$ neither satisfies the ascending nor the descending chain condition. Let 
$\Lambda$ be the Kronecker algebra, this is the path algebra of the quiver

$$
a \longleftarrow b
$$

The $\Lambda$-modules are also called Kronecker modules (basic facts concerning the Kronecker modules will be recalled in Sect. 14). Let $Y=S(b)$, the simple injective module.

We denote by $Q_{n}$ the indecomposable preinjective module of length $2 n+1$ (thus $\left.Q_{0}=S(b)=Q(b), Q_{1}=Q(a)\right)$. There is a chain of epimorphisms

$$
\cdots \longrightarrow Q_{2} \stackrel{f_{2}}{\longrightarrow} Q_{1} \stackrel{f_{1}}{\longrightarrow} Q_{0}=Y
$$

thus we have the descending chain

$$
\cdots\left[f_{1} f_{2} f_{3}\right\rangle<\left[f_{1} f_{2}\right\rangle<\left[f_{1}\right\rangle<\left[1_{Y}\right\rangle
$$

in $[\rightarrow Y\rangle$.

Here, we can assume that all the kernels $f_{n}: Q_{n} \rightarrow Q_{n-1}$ are equal to $R$, where $R$ is a fixed indecomposable module of length 2. Also, if the ground field $k$ is infinite, then there is such a chain of epimorphisms such that all the kernels are pairwise different and of length 2 . In the first case, the kernels of the maps $f_{1} f_{2} \cdots f_{n}$ are all indecomposable (namely of the form $R[n]$ for $n \in \mathbb{N}$ ), in the second, they are direct sums of pairwise non-isomorphic modules of length 2 .

In order to look at the ascending chain condition, let $P_{n}$ be indecomposable preprojective of length $2 n+1$. For $i \geq 1$, there are epimorphisms $f_{i}: P_{i} \rightarrow Y$ and monomorphisms $u_{i}: P_{i} \rightarrow P_{i+1}$ such that $f_{i+1} u_{i}=f_{i}$. Thus, there is a commutative diagram of maps

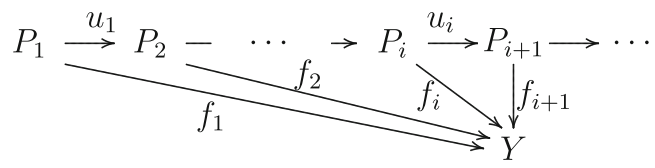

and we obtain an ascending chain in $[\rightarrow Y\rangle$.

$$
\left[f_{1}\right\rangle<\left[f_{2}\right\rangle<\left[f_{3}\right\rangle<\cdots
$$

Thus, looking at the sequence of maps

$$
P_{1} \stackrel{u_{1}}{\longrightarrow} P_{2} \stackrel{u_{2}}{\longrightarrow} \cdots \longrightarrow Y
$$

we obtain an ascending chain in $[\rightarrow Y\rangle$.

$$
\left[f_{1}\right\rangle<\left[f_{2}\right\rangle<\left[f_{3}\right\rangle<\cdots .
$$

There are similar chains in $[\rightarrow Y\rangle$ consisting of elements $[f: X \rightarrow Y\rangle$ with $X$ regular Kronecker modules. 
Remark If we fix $Y$ (as we usually do), we may consider the class of right minimal morphisms $f: X \rightarrow Y$ as the objects of a category, with maps from $(f: X \rightarrow Y)$ to $\left(f^{\prime}: X^{\prime} \rightarrow Y\right.$ ) being given by the maps $h: X \rightarrow X^{\prime}$ such that $f=f^{\prime} h$. According to Proposition 2.1, this category is a groupoid (this means that all morphisms are isomorphisms), and any connected component of the category is just the class of all the right minimal maps which belong to a right equivalence class. If we work with a skeleton of the category $\bmod \Lambda$, then we only have to consider the sets

$$
\operatorname{r-Aut}(f)=\{h \in \operatorname{End}(X) \mid f h=h\},
$$

this is a subgroup of $\operatorname{Aut}(X)$; we may call it the right automorphism group of $f$. The classification problem for the right minimal maps ending in $Y$ is divided in this way into two problems: to determine, on the one hand, the structure of the right factorization lattice $[\rightarrow Y\rangle$ and, on the other hand, to determine r-Aut $(f)$ for every right minimal map $f$ ending in $Y$. This provides a nice separation of the local symmetries and the global directedness, as mentioned at the beginning of the paper.

\section{Morphisms determined by modules: Auslander's First Theorem}

Here is the decisive definition. Let $f: X \rightarrow Y$ be a morphism and $C$ a module. Then $f$ is said to be right $C$-determined (or right determined by $C$ ) provided the following condition is satisfied: given any morphism $f^{\prime}: X^{\prime} \rightarrow Y$ such that $f^{\prime} \phi$ factors through $f$ for all $\phi: C \rightarrow X^{\prime}$, then $f^{\prime}$ itself factors through $f$. Thus one deals with the following diagrams:
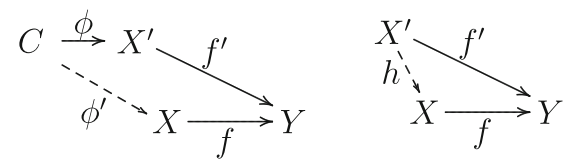

The existence of the dashed arrow $\phi^{\prime}$ on the left for all possible maps $\phi: C \rightarrow X^{\prime}$ shall imply the existence of the dashed arrow $h$ on the right (of course, the converse implication always holds true: if $f^{\prime}=f h$ for some morphism $h$, then $f^{\prime} \phi=f(h \phi)$ for all morphisms $\phi: C \rightarrow X^{\prime}$ ).

Proposition 3.1 Let $: X \rightarrow Y$ be a morphism, let $C, C^{\prime}$ be modules.

(a) Assume that add $C=$ add $C^{\prime}$. Then $f$ is right $C$-determined if and only if $f$ is right $C^{\prime}$-determined.

(b) If $f$ is right $C$-determined, then $f$ is also right $\left(C \oplus C^{\prime}\right)$-determined.

Proof Trivial verification.

We denote by ${ }^{C}[\rightarrow Y\rangle$ the set of the right equivalence classes of the morphisms ending in $Y$ which are right $C$-determined. We will see below that also ${ }^{C}[\rightarrow Y\rangle$ is a lattice, thus we call it the right $C$-factorization lattice for $Y$.

Note that ${ }^{C}[\rightarrow Y\rangle$ is usually not closed under predecessors or successors inside $[\rightarrow Y\rangle$. But there is the following important property: 
Proposition 3.2 The subset ${ }^{C}[\rightarrow Y\rangle$ of $[\rightarrow Y\rangle$ is closed under meets.

Proof Let $f_{1}: X_{1} \rightarrow Y$ and $f_{2}: X_{2} \rightarrow Y$ be right $C$-determined. As we know, the meet of $\left[f_{1}\right\rangle$ and $\left[f_{2}\right\rangle$ is given by forming the pullback of $f_{1}$ and $f_{2}$. Thus assume that $X$ is the pullback with maps $g_{1}: X \rightarrow X_{1}$ and $g_{2}: X \rightarrow X_{2}$ and let $f=f_{1} g_{1}=f_{2} g_{2}$. We want to show that $f$ is right $C$-determined. Thus, assume that there is given $f^{\prime}: X^{\prime} \rightarrow Y$ such that for any $\phi: C \rightarrow X^{\prime}$, there exists $\phi^{\prime}: C \rightarrow X$ such that $f^{\prime} \phi=f \phi^{\prime}$. Then we see that for any $\phi: C \rightarrow X^{\prime}$, we have $f^{\prime} \phi=f \phi=f_{1}\left(g_{1} \phi\right)$, thus $f^{\prime} \phi$ factors through $f_{1}$. Since $f_{1}$ is right $C$-determined, it follows that $f^{\prime}$ factors through $f_{1}$, say $f^{\prime}=f_{1} h_{1}$ for some $h_{1}: X^{\prime} \rightarrow X_{1}$. Similarly, for any $\phi: C \rightarrow X^{\prime}$, the morphism $f^{\prime} \phi$ factors through $f_{2}$ and therefore $f^{\prime}=f_{2} h_{2}$ for some $h_{2}: X^{\prime} \rightarrow X_{2}$. Now $f_{1} h_{1}=f^{\prime}=f_{2} h_{2}$ implies that there is $h: X^{\prime} \rightarrow X$ such that $g_{1} h=h_{1}$, and $g_{2} h=h_{2}$. Thus $f^{\prime}=f_{1} h_{1}=f_{1} g_{1} h=f h$ shows that $f^{\prime}$ factors through $f$.

We should stress that ${ }^{C}[\rightarrow Y\rangle$ usually is not closed under joins, see the examples at the end of the section. One of these examples is chosen in order to convince the reader that this is not at all a drawback, but an important feature if we want to work with lattices of finite height.

Theorem 3.3 (Auslander's First Theorem) For any $\Lambda$-module $Y$, one has

$$
[\rightarrow Y\rangle=\bigcup_{C}{ }^{C}[\rightarrow Y\rangle
$$

where $C$ runs through all the $\Lambda$-modules (or just through representatives of all multiplicity-free $\Lambda$-modules) and this is a filtered union of meet-semilattices.

By definition, the sets ${ }^{C}[\rightarrow Y\rangle$ are subsets of $[\rightarrow Y\rangle$. By Proposition 3.1(a), we know that ${ }^{C}[\rightarrow Y\rangle$ only depends on add $C$, thus we may restrict to look at representatives of multiplicity-free $\Lambda$-modules $C$. Proposition 3.1(b) asserts that both ${ }^{C}[\rightarrow Y\rangle$ and $C^{\prime}[\rightarrow Y\rangle$ are contained in $C \oplus C^{\prime}[\rightarrow Y\rangle$, thus we deal with a filtered union. According to Proposition 3.2, we deal with embeddings of meet-semilattices. The essential assertion of Theorem 3.3 is that any morphism is right determined by some module, the usual formulation of Auslander's First Theorem. A discussion of this assertion and its proof follows.

There is a precise formula which yields for $f$ the smallest possible module $C(f)$ which right determines $f$. We will call it the minimal right determiner of $f$, any other right determiner of $f$ will have $C(f)$ as a direct summand.

We need another definition. An indecomposable projective module $P$ is said to almost factor through $f$, provided there is a commutative diagram of the following form

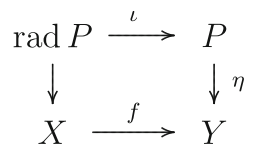

where $\iota$ is the inclusion map, such that the image of $\eta$ is not contained in the image of $f$. Let us mention the following: If the indecomposable projective module $P$ almost 
factors through $f$, then $P / \operatorname{rad} P$ embeds into the cokernel $\operatorname{Cok}(f)$. Namely, given a map $\eta: P \rightarrow Y$ such that the image of $\eta$ is not contained in the image of $f$, as well as the commutative diagram above, we may complete the diagram by adding the cokernels of the horizontal maps:

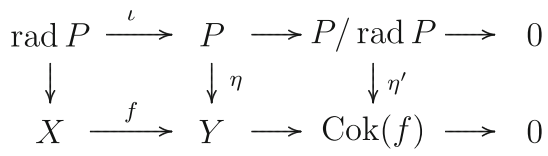

Since the image of $\eta$ is not contained in the image of $f$, we see that $\eta^{\prime}$ is non-zero, thus $P / \operatorname{rad} P$ is a submodule of $\operatorname{Cok}(f)$.

Theorem 3.4 (Determiner formula of Auslander-Reiten-Smalø) Let $f$ be a morphism ending in $Y$. Let $C(f)$ be the direct sum of the indecomposable modules of the form $\tau^{-} K$, where $K$ is an indecomposable direct summand of the intrinsic kernel of $f$ and of the indecomposable projective modules which almost factor through $f$, one from each isomorphism class. Then $f$ is right $C$-determined if and only if $C(f) \in \operatorname{add} C$.

The theorem suggests to call $C(f)$ the minimal right determiner of $f$. For the proof of Theorem 3.4, see [5] and also [38].

Corollary 3.5 Any morphism $f$ is right $C$-determined by some $C$, for example by the module

$$
\tau^{-} \operatorname{Ker}(f) \oplus P(\operatorname{soc} \operatorname{Cok}(f))
$$

Proof We have to show that $C(f)$ is a direct summand of $\tau^{-} \operatorname{Ker}(f) \oplus P(\operatorname{soc} \operatorname{Cok}(f))$. The intrinsic kernel of $f$ is a direct summand of $\operatorname{Ker}(f)$, thus if $K$ is an indecomposable direct summand of the intrinsic kernel of $f$, then $\tau^{-} K$ is a direct summand of $\tau^{-} \operatorname{Ker}(f)$. Now assume that $S$ is a simple module such that $P(S)$ almost factors through $f$. Then $S$ is a submodule of $\operatorname{Cok}(f)$, thus $P(S)$ is a direct summand of $P(\operatorname{soc} \operatorname{Cok}(f))$.

Corollary 3.6 (Auslander) The module $\tau^{-} \operatorname{Ker}(f) \oplus \Lambda$ right determines $f$.

Corollary 3.7 Let $P$ be a projective module and $f: X \rightarrow Y$ a right minimal morphism. Then $f$ is right $P$-determined if and only if $f$ is a monomorphism and the socle of the cokernel of $f$ is generated by $P$.

Proof This is an immediate consequence of the determiner formula: First, assume that $f$ is right $P$-determined. Then the intrinsic kernel of $f$ has to be zero. Since we assume that $f$ is right minimal, $f$ must be a monomorphism. If $S$ is a simple submodule of the cokernel of $f$, then $P(S)$ almost factors through $f$, thus $P(S)$ is a direct summand of $P$. This shows that the socle of the cokernel of $f$ is generated by $P$. Conversely, assume that $f$ is a monomorphism and the socle of the cokernel of $f$ is generated by $P$. Since $f$ is a monomorphism, $C(f)$ is the direct sum of all indecomposable projective modules $P^{\prime}$ which almost factor through $f$. Such a module $P^{\prime}$ is the projective cover 
of a simple submodule of $\operatorname{Cok}(f)$. Since $P$ generates the socle of the cokernel of $f$, it follows that $P^{\prime}$ is a direct summand of $P$. Thus $C(f)$ is in add $P$, therefore $f$ is right $P$-determined.

Corollary 3.8 A right minimal morphism $f: X \rightarrow Y$ is a monomorphism if and only if it is right $\Lambda$-determined.

Example 3 The subset ${ }^{C}[\rightarrow Y\rangle$ of $[\rightarrow Y\rangle$ is usually not closed under joins, as the following example shows: Let $\Lambda$ be the path algebra of the quiver of type $\mathbb{A}_{3}$ with two sources, namely of<smiles>[Mg][TeH]</smiles>

Let $f_{i}: P\left(b_{i}\right) \rightarrow Q(a)$ be non-zero maps for $i=1,2$, these are monomorphisms, thus they are right $\Lambda$-determined. The join of $\left[f_{1}\right\rangle$ and $\left[f_{2}\right\rangle$ in $[\rightarrow Y\rangle$ is given by the map $\left[f_{1}, f_{2}\right]: P\left(b_{1}\right) \oplus P\left(b_{2}\right) \rightarrow Q(a)$. Clearly, this map is right minimal, but it is not injective. Thus $\left[f_{1}, f_{2}\right]$ is not right $\Lambda$-determined.

Example 4 This example indicates that in general, it may not be advisable to ask for closure under joins. Consider again the Kronecker algebra $\Lambda$ as exhibited in example 2. Let $Y=Q(a)$ and $C=\Lambda$, thus all the maps in ${ }^{C}[\rightarrow Y\rangle$ are given by inclusion maps $f: X \rightarrow Y$, where $X$ is a submodule of $Y$. In fact, we may identify ${ }^{C}[\rightarrow Y\rangle$ with the submodule lattice of $Y$, it is a lattice of height 3 which looks as follows:
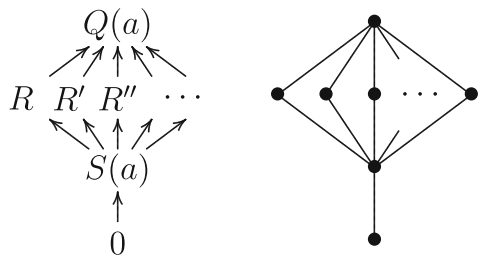

Here, the modules $R, R^{\prime}, R^{\prime \prime}, \ldots$ are the indecomposable representations of length 2 , one from each isomorphism class and all the arrows are inclusion maps.

The join in ${ }^{C}[\rightarrow Y\rangle$ of two different maps $f_{1}, f_{2}$ in the height 2 layer is just the identity map $Y \rightarrow Y$, whereas the join of $f_{1}, f_{2}$ in $[\rightarrow Y\rangle$ is the direct sum map $\left[f_{1}, f_{2}\right]: R_{1} \oplus R_{2} \rightarrow Y$. More generally, if there are given $n$ pairwise different regular modules $R_{1}, \ldots, R_{n}$ of length 2 with inclusion maps $f_{i}: R_{i} \rightarrow Y$, then the join in $[\rightarrow Y\rangle$ is the direct sum map $\left[f_{1}, \ldots, f_{n}\right]: R_{1} \oplus \cdots \oplus R_{n} \rightarrow Y$. Let us stress that all these direct sum maps are right minimal (thus here we deal with a cofork as defined in Sect. 13). Thus, if the base field $k$ is infinite, the smallest subposet of $[\rightarrow Y\rangle$ closed under meets and joins and containing the inclusion maps $R \rightarrow Y$ with $R$ regular of length 2 will have infinite height.

Proposition 3.9 Let $f: X \rightarrow Y$ be a morphism. If $C^{\prime}$ is an indecomposable direct summand of $C(f)$, then $\operatorname{Hom}\left(C^{\prime}, Y\right) \neq 0$. 
Proof By definition, there are two kinds of indecomposable direct summands of $C(f)$, the non-projective ones are of the form $\tau^{-} K^{\prime}$, where $K^{\prime}$ is an indecomposable direct summand of the intrinsic kernel of $f$, the remaining ones are the indecomposable projective modules which almost factor through $f$. Of course, if $P$ is an indecomposable projective module which almost factors through $f$, then $\operatorname{Hom}(P, Y) \neq 0$.

Thus, we have to consider a module of the form $C^{\prime}=\tau^{-} K^{\prime}$ with $K^{\prime}$ an indecomposable direct summand of the intrinsic kernel $K$ of $f$ and note that $K^{\prime}$ cannot be injective. Let us denote by $u: K \rightarrow X$ and $u^{\prime}: K^{\prime} \rightarrow K$ the inclusion maps. Since $u^{\prime}$ is split mono, there is $r: K \rightarrow K^{\prime}$ with $r u^{\prime}=1_{K^{\prime}}$. Let

$$
0 \longrightarrow K^{\prime} \stackrel{\mu}{\longrightarrow} M \stackrel{\epsilon}{\longrightarrow} C^{\prime} \longrightarrow 0
$$

be the Auslander-Reiten sequence starting with $K^{\prime}$. Since the composition $u u^{\prime}$ is not split mono, there is a map $\phi: M \rightarrow X$ with $\phi \mu=u u^{\prime}$. Thus, there is the following commutative diagram with exact rows

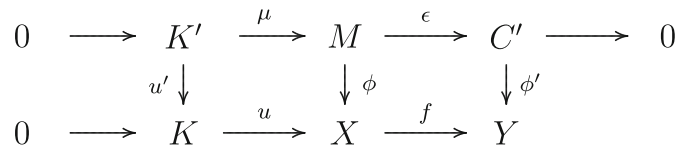

If we assume that $\phi^{\prime}=0$, then $f \phi=0$, thus $\phi$ factors through the kernel of $f$, say $\phi=u \phi^{\prime \prime}$. Consequently, $u u^{\prime}=\phi \mu=u \phi^{\prime \prime} \mu$. But $u$ is injective, thus $u^{\prime}=\phi^{\prime \prime} \mu$ and therefore $1_{K^{\prime}}=r u^{\prime}=r \phi^{\prime \prime} \mu$. But this means that $\mu$ is split mono, a contradiction. It follows that $\phi^{\prime} \neq 0$, thus $\operatorname{Hom}\left(C^{\prime}, Y\right) \neq 0$.

Remark Proposition 3.9 asserts that all the indecomposable direct summands $C^{\prime}$ of the minimal right determiner $C(f)$ of a map $f: X \rightarrow Y$ satisfy $\operatorname{Hom}\left(C^{\prime}, Y\right) \neq 0$. Actually, according to [5], Proposition XI.2.4 (see also [38]), such a module $C^{\prime}$ is equipped with a distinguished non-zero map $C^{\prime} \rightarrow Y$ which is said to "almost factor through" $f$. At the beginning of this section we gave a corresponding definition in the special case when $C^{\prime}$ is projective. See also the Remark 3 at the end of Sect. 4.

\section{The Auslander bijection. Auslander's Second Theorem}

Let $C, Y$ be objects. Let $\Gamma(C)=\operatorname{End}(C)^{\text {op }}$. We always will consider $\operatorname{Hom}(C, Y)$ as a $\Gamma(C)$-module. For any module $M$, we denote by $\mathcal{S} M$ the set of all submodules (it is a lattice with respect to intersection and sum of submodules).

Define

$$
\eta_{C Y}: \bigcup_{X} \operatorname{Hom}(X, Y) \longrightarrow \mathcal{S}(\operatorname{Hom}(C, Y))
$$

by $\eta_{C Y}(f)=\operatorname{Im} \operatorname{Hom}(C, f)=f \cdot \operatorname{Hom}(C, X)$ for $f: X \rightarrow Y$ (note that $f$. $\operatorname{Hom}(C, X)$ clearly is a $\Gamma(C)$-submodule).

Here is a reformulation of the definition of $\eta_{C Y}$. 
Proposition 4.1 Let $f: X \rightarrow Y$. Then $\eta_{C Y}(f)$ is the set of all $h \in \operatorname{Hom}(C, Y)$ which factor through $f$. This subset of $\operatorname{Hom}(C, Y)$ is a $\Gamma(C)$-submodule.

Proof We have mentioned already, that $\eta_{C Y}(f)$ is a $\Gamma(C)$-submodule of $\operatorname{Hom}(C, Y)$. Also, if $h \in \eta_{C Y}(f)=f \operatorname{Hom}(C, X)$, then $h$ factors through $f$. And conversely, if $h$ factors through $f$, then $h$ belongs to $f \operatorname{Hom}(C, X)=\eta_{C Y}(f)$.

Lemma 4.2 If $X=X_{0} \oplus X_{1}$ and $f\left(X_{0}\right)=0$, then $\eta_{C Y}(f)=\eta_{C Y}\left(f \mid X_{1}\right)$.

Proof Let $X=X_{0} \oplus X_{1}$ and write $f=\left[\begin{array}{ll}f_{0} & f_{1}\end{array}\right]=\left[\begin{array}{ll}0 & f_{1}\end{array}\right]$ with $f_{i}: X_{i} \rightarrow Y$. Then, for $\phi_{i}: C \rightarrow X_{i}$, we have $\left[\begin{array}{ll}0 & f_{1}\end{array}\right]\left[\begin{array}{l}\phi_{0} \\ \phi_{1}\end{array}\right]=f_{1} \phi_{1}$. Thus

$$
\eta_{C Y}(f)=f \operatorname{Hom}\left(C, X_{0} \oplus X_{1}\right)=f_{1} \operatorname{Hom}\left(C, X_{1}\right)=\eta_{C Y}\left(f_{1}\right) .
$$

In particular: If $f_{1}$ is a right minimal version of $f$, then $\eta_{C Y}(f)=\eta_{C Y}\left(f_{1}\right)$. Thus, $\eta_{C Y}$ is constant on right equivalence classes and we can define $\eta_{C Y}([f\rangle)=\eta_{C Y}(f)$. We obtain in this way a map

$$
\eta_{C Y}:[\rightarrow Y\rangle \rightarrow \mathcal{S}(\operatorname{Hom}(C, Y))
$$

Of special interest is the restriction of $\eta_{C Y}$ to ${ }^{C}[\rightarrow Y\rangle$.

Proposition 4.3 Let $C, Y$ be modules. The map

$$
\eta_{C Y}:{ }^{C}[\rightarrow Y\rangle \rightarrow \mathcal{S} \operatorname{Hom}(C, Y)
$$

is injective and preserves meets. As a consequence, it preserves and reflects the ordering.

Proof A trivial verification: First, let us show that $\eta_{C Z}$ is injective. Consider maps $f: X \rightarrow Y$ and $f^{\prime}: X^{\prime} \rightarrow Y$ such that $f \operatorname{Hom}(C, X)=f^{\prime} \operatorname{Hom}\left(C, X^{\prime}\right)$. Since $f$ is right $C$-determined and $f^{\prime} \operatorname{Hom}\left(C, X^{\prime}\right) \subseteq f \operatorname{Hom}(C, X)$, we see that $f^{\prime} \in$ $f \operatorname{Hom}\left(X^{\prime}, X\right)$. Since $f^{\prime}$ is right $C$-determined and $f \operatorname{Hom}(C, X) \subseteq f^{\prime} \operatorname{Hom}\left(C, X^{\prime}\right)$, we see that $f \in f \operatorname{Hom}\left(X, X^{\prime}\right)$. But this means that $f^{\prime} \preceq f \preceq f^{\prime}$, thus $[f\rangle=\left[f^{\prime}\right\rangle$.

Next, consider the following pullback diagram

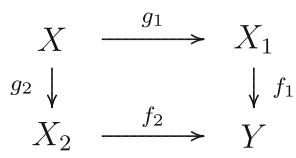

such that $f_{1}, f_{2}$ both are right $C$-determined. Let $f=f_{1} g_{1}=f_{2} g_{2}$, thus the meet of $\left[f_{1}\right\rangle$ and $\left[f_{2}\right\rangle$ is $[f\rangle$. Now $f=f_{1} g_{1}$ shows that $f \operatorname{Hom}(C, X) \subseteq f_{1} \operatorname{Hom}\left(C, X_{1}\right)$. 
Similarly, $f=f_{2} g_{2}$ shows that $f \operatorname{Hom}(C, X) \subseteq f_{2} \operatorname{Hom}\left(C, X_{2}\right)$. Both assertions together yield

$$
f \operatorname{Hom}(C, X) \subseteq f_{1} \operatorname{Hom}\left(C, X_{1}\right) \cap f_{2} \operatorname{Hom}\left(C, X_{2}\right)
$$

Conversely, take an element in $f_{1} \operatorname{Hom}\left(C, X_{1}\right) \cap f_{2} \operatorname{Hom}\left(C, X_{2}\right)$, say $f_{1} \phi_{1}=f_{2} \phi_{2}$ with $\phi_{i}: C \rightarrow X_{i}$, for $i=1,2$. The pullback property yields a morphism $\phi: C \rightarrow X$ such that $g_{i} \phi=\phi_{i}$ for $i=1,2$. Therefore $f_{1} \phi_{1}=f_{1} g_{1} \phi=f \phi$ belongs to $f \operatorname{Hom}(C, X)$. Thus,

$$
f_{1} \operatorname{Hom}\left(C, X_{1}\right) \cap f_{2} \operatorname{Hom}\left(C, X_{2}\right) \subseteq f \operatorname{Hom}(C, X),
$$

and therefore

$$
f_{1} \operatorname{Hom}\left(C, X_{1}\right) \cap f_{2} \operatorname{Hom}\left(C, X_{2}\right)=f \operatorname{Hom}(C, X) .
$$

In general, assume that $L, L^{\prime}$ are posets with meets and $\eta: L \rightarrow L^{\prime}$ is a settheoretical map which preserves meets. Then $a \leq b$ in $L$ implies $\eta(a) \leq \eta(b)$ in $L^{\prime}$. Namely, $a \leq b$ gives $a \wedge b=a$, thus $\eta(a) \wedge \eta(b)=\eta(a)$ and therefore $\eta(a) \leq \eta(b)$. Conversely, if $a, b$ are arbitrary elements in $L$ with $\eta(a) \leq \eta(b)$, let $c=a \wedge b$. Then $\eta(c)=\eta(a) \wedge \eta(b)=\eta(a)$. Thus, if $\eta$ is injective, then $c=a$, and $a \wedge b=a$ implies $a \leq b$. Altogether, we see that $\eta_{C Y}$ preserves and reflects the ordering.

Auslander's Second Theorem (as established in [2]) asserts:

Theorem 4.4 (Auslander's Second Theorem) The map

$$
\eta_{C Y}:[\rightarrow Y\rangle \rightarrow \mathcal{S}(\operatorname{Hom}(C, Y))
$$

is surjective.

Altogether we see: The map $\eta_{C Y}$ defined by $\eta_{C Y}(f)=\operatorname{Im} \operatorname{Hom}(C, f)$ yields a lattice isomorphism

$$
\eta_{C Y}:{ }^{C}[\rightarrow Y\rangle \longrightarrow \mathcal{S} \operatorname{Hom}(C, Y)
$$

Better: The composition

$$
{ }^{C}[\rightarrow Y\rangle \quad \subseteq \quad[\rightarrow Y\rangle \quad \stackrel{\eta_{C Y}}{\longrightarrow} \mathcal{S}_{\Gamma(C)} \operatorname{Hom}(C, Y)
$$

of the inclusion map and the map $\eta_{C Y}$ defined by $\eta_{C Y}(f)=\operatorname{Im} \operatorname{Hom}(C, f)$ is a lattice isomorphism.

Convention. In the following, several examples of Auslander bijections will be presented. When looking at the submodule lattice $\mathcal{S} M$ of a module $M$, we usually will 
mark (some of) the elements of $\mathcal{S} M$ by bullets $\bullet$ and connect comparable elements by a solid lines. Here, going upwards corresponds to the inclusion relation.

For the corresponding lattices ${ }^{C}[\rightarrow Y\rangle$, we often will mark an element $[f: X \rightarrow Y\rangle$ (with $f$ a right minimal map) by just writing $X$ and we will connect neighboring pairs $[f: X \rightarrow Y\rangle \leq\left[f^{\prime}: X^{\prime} \rightarrow Y\right\rangle$ by drawing an (upwards) arrow $X \rightarrow Y$. On the other hand, sometimes it seems to be more appropriate to refer to the right minimal map $f: X \rightarrow Y$ with kernel $K^{\prime}$ and image $Y^{\prime}$ by using the short exact sequence notation $K^{\prime} \rightarrow X \rightarrow Y^{\prime}$.

Note that the lattice $\mathcal{S} \operatorname{Hom}(C, Y)$ has two distinguished elements, namely $\operatorname{Hom}(C, Y)$ itself as well as its zero submodule. Under the bijection $\eta_{C Y}$ the total submodule $\operatorname{Hom}(C, Y)$ corresponds to the identity map $1_{Y}$ of $Y$, this is not at all exciting. But of interest seem to be the maps in $\eta_{C Y}^{-1}(0)$, we will discuss them in this will be discussed in Proposition 5.5

The special case $C={ }_{\Lambda} \Lambda$. It is worthwhile to draw the attention on the special case when $C={ }_{\Lambda} \Lambda$.

Proposition 4.5 The special case of the Auslander bijection $\eta_{\Lambda Y}$ is the obvious identification of both ${ }^{\Lambda}[\rightarrow Y\rangle$ and $\mathcal{S} \operatorname{Hom}(\Lambda, Y)$ with $\mathcal{S} Y$.

Proof First, consider ${ }^{\Lambda}[\rightarrow Y\rangle$ : The determiner formula asserts: a right minimal morphism is right $\Lambda$-determined if and only if it is a monomorphism. Thus ${ }^{\Lambda}[\rightarrow Y\rangle$ is just the set of right equivalence classes of monomorphisms ending in $Y$, and the map $f \mapsto \operatorname{Im}(f)$ yields an identification between the set of right equivalence classes of monomorphisms ending in $Y$ and the submodules of $\Lambda$.

Next, we deal with $\mathcal{S} \operatorname{Hom}(\Lambda, Y)$. Note that $\Gamma\left({ }_{\Lambda} \Lambda\right)=\operatorname{End}(\Lambda \Lambda)^{\mathrm{op}}=\Lambda$ and there is a canonical identification $\epsilon: \operatorname{Hom}(\Lambda, Y) \simeq Y$ (given by $\epsilon(h)=h(1)$ for $h \in \operatorname{Hom}(\Lambda, Y)$ ), thus $\mathcal{S} \epsilon: \mathcal{S} \operatorname{Hom}(\Lambda, Y) \simeq \mathcal{S} Y$ (with $\mathcal{S} \epsilon(U)=\{h(1) \mid h \in U\}$ for $U$ a submodule of $\operatorname{Hom}(\Lambda, Y)$ ).

The Auslander bijection $\eta_{\Lambda, Y}$ attaches to $f: X \rightarrow Y$ the submodule $f \operatorname{Hom}(\Lambda, X)$ and there is the following commutative diagram:
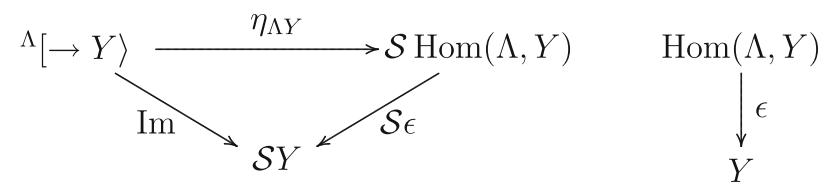

Namely, for $f: X \rightarrow Y$ we have

$$
\begin{aligned}
(\mathcal{S} \epsilon) \eta_{\Lambda Y}(f) & =(\mathcal{S} \epsilon)(f \operatorname{Hom}(\Lambda, X)) \\
& =\{f h(1) \mid h \in \operatorname{Hom}(\Lambda, X)\}=\{f(x) \mid x \in X\}=\operatorname{Im}(f) .
\end{aligned}
$$

As a consequence, we see that all possible submodule lattices $\mathcal{S Y}$ occur as images under the Auslander bijections. This assertion can be strengthened considerably, as we want to show now.

By definition, an artin algebra $\Lambda$ is an artin $k$-algebra for some commutative artinian ring $k$ (this means that $\Lambda$ is a $k$-algebra and that it is finitely generated as a $k$-module). Such an algebra is said to be strictly wild (or better strictly $k$-wild), provided for any 
artin $k$-algebra $\Gamma$, there is a full exact embedding $\bmod \Gamma \rightarrow \bmod \Lambda$. If $M$ is a $\Lambda$ module and $M^{\prime}$ is a $\Lambda^{\prime}$-module, a semilinear isomorphism from $M$ to $M^{\prime}$ is a pair $(\alpha, f)$, where $\alpha: \Lambda \rightarrow \Lambda^{\prime}$ is an algebra isomorphism, and $f: M \rightarrow M^{\prime}$ is an isomorphism of abelian groups such that $f(\lambda m)=\alpha(\lambda) f(m)$ for all $\lambda \in \Lambda$ and $m \in M$. It is clear that any semilinear isomorphism from $M$ to $M^{\prime}$ induces a lattice isomorphism $\mathcal{S} M \rightarrow \mathcal{S} M^{\prime}$.

Proposition 4.6 Let $\Lambda$ be an artin k-algebra which is strictly $k$-wild. Let $\Gamma$ be an artin $k$-algebra and $M a \Gamma$-module. Then there are $\Lambda$-modules $C, Y$ such that the $\Gamma(C)$-module $\operatorname{Hom}(C, Y)$ is semilinearly isomorphic to $M$. Thus there is a lattice isomorphism ${ }^{C}[\rightarrow Y\rangle \rightarrow \mathcal{S} M$.

Proof Let $F: \bmod \Gamma \rightarrow \bmod \Lambda$ be a full embedding (we do not need that it is exact). Let $C=F(\Gamma \Gamma)$ and $Y=F(M)$. Let $\alpha: \Gamma=\operatorname{End}\left({ }_{\Gamma} \Gamma\right)^{\text {op }} \rightarrow \operatorname{End}(C)^{\mathrm{op}}=\Gamma(C)$ as well as $f: M=\operatorname{Hom}(\Gamma \Gamma, M) \rightarrow \operatorname{Hom}(C, M)$ both be given by applying the functor $F$. Since $F$ is a full embedding, $\alpha$ is an algebra isomorphism and $f$ is an isomorphism of abelian groups. The functoriality of $F$ asserts that we also have $f(\gamma m)=\alpha(\gamma) f(m)$ for all $\gamma \in \Gamma$ and $m \in M$. This shows that the pair $(\alpha, f)$ is a semilinear isomorphism.

Remark If $F: \bmod \Gamma \rightarrow \bmod \Lambda$ is a full embedding functor, and $C, Y$ are $\Gamma$-modules, then we obtain a bijection

$$
{ }^{C}[\rightarrow y\rangle \stackrel{\eta_{C Y}}{\longrightarrow} \mathcal{S} \operatorname{Hom}(C, Y) \stackrel{F}{\longrightarrow} \mathcal{S} \operatorname{Hom}(F(C), F(Y)) \stackrel{\eta_{F(C), F(Y)}^{-1}}{\longrightarrow} F(C)[\rightarrow F(Y),
$$

but even if $F$ is exact, such a bijection will not be given by applying directly $F$. Namely, if $f: X \rightarrow Y$ is right minimal and right $C$-determined, then the kernel of $f$ belongs to add $\tau C$, thus the kernel of $F(f)$ belongs to add $F(\tau C)$, whereas the intrinsic kernel of any right $F(C)$-determined map has to belong to add $\tau F(C)$ and the $\Lambda$-modules $F(\tau C)$ and $\tau F(C)$ may be very different, as the obvious embeddings of the category of $n$-Kronecker modules into the category of $(n+1)$-Kronecker modules (using for one arrow the zero map) show.

Note that under a full exact embedding functor $F: \bmod \Gamma \rightarrow \bmod \Lambda$, submodule lattices are usually not preserved: given a $\Gamma$-module $M$, the functor $F$ yields an embedding of $\mathcal{S}\left({ }_{\Gamma} M\right)$ into $\mathcal{S}\left({ }_{\Lambda} F(M)\right)$, but usually this is a proper embedding. Actually, for any finite-dimensiona algebra $\Lambda$, there are submodule lattices $\mathcal{S}\left({ }_{\Gamma} M\right)$ which cannot be realized as the submodule lattice of any $\Lambda$-module. Namely, assume that the length of the indecomposable projective $\Lambda$-modules is bounded by $t$ and take a finite-dimensional algebra $\Gamma$ with a local $\Gamma$-module $M$ of length $t+1$. Then $\mathcal{S} M$ is a modular lattice of height $t+1$ with a unique element of height $t$ (the radical of the module $M$ ). If $\mathcal{S}\left({ }_{\Lambda} Y\right)$ is of the form $\mathcal{S} M$, then $Y$ has to be a local $\Lambda$-module of length $t+1$, thus a factor module of an indecomposable projective $\Lambda$-module. But by assumption, the indecomposable projective $\Lambda$-modules have length at most $t$.

Remark 1 Let $Y=\bigoplus Y_{i}$, then the subsets $\operatorname{Hom}\left(C, Y_{i}\right)$ of $\operatorname{Hom}(C, Y)$ are actually $\Gamma(C)$-submodules and there is an isomorphism of $\Gamma(C)$-modules $\operatorname{Hom}(C, Y) \simeq$ $\bigoplus_{i} \operatorname{Hom}\left(C, Y_{i}\right)$. Thus $\eta_{C Y}$ maps the lattice ${ }^{C}[\rightarrow Y\rangle$ bijectively onto the submodule 
lattice $\mathcal{S}\left(\bigoplus_{i} \operatorname{Hom}\left(C, Y_{i}\right)\right.$. The lattice $\mathcal{S}\left(\bigoplus_{i} \operatorname{Hom}\left(C, Y_{i}\right)\right.$ contains $\prod_{i} \mathcal{S} \operatorname{Hom}\left(C, Y_{i}\right)$ as a sublattice and both have the same height. However, $\prod_{i} \mathcal{S} \operatorname{Hom}\left(C, Y_{i}\right)$ may be a proper sublattice of $\mathcal{S}\left(\bigoplus_{i} \operatorname{Hom}\left(C, Y_{i}\right)\right.$, since isomorphisms of subfactors of the various modules $\operatorname{Hom}\left(C, Y_{i}\right)$ yield diagonals in $\mathcal{S}\left(\bigoplus_{i} \operatorname{Hom}\left(C, Y_{i}\right)\right.$.

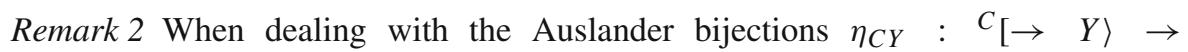
$\mathcal{S} \operatorname{Hom}(C, Y)$, we always can assume that $C$ is multiplicity-free and supporting, here supporting means that $\operatorname{Hom}\left(C_{i}, Y\right) \neq 0$ for any indecomposable direct summand $C_{i}$ of $C$. Namely, let $C^{\prime}$ be the direct sum of all indecomposable direct summands $C_{i}$ of $C$ with $\operatorname{Hom}\left(C_{i}, Y\right) \neq 0$, one from each isomorphism class. Then, on the one hand, ${ }^{C}[\rightarrow Y\rangle={ }^{\prime}[\rightarrow Y\rangle$ (since a map $f$ ending in $Y$ is right $C$-determined if and only if it is right $C^{\prime}$-determined. On the other hand, there is an idempotent $e \in \Gamma(C)$ such that $e \Gamma(C) e=\Gamma\left(C^{\prime}\right)$ and $e \operatorname{Hom}(C, Y) e=\operatorname{Hom}\left(C^{\prime}, Y\right)$, and there is a lattice isomorphism $\mathcal{S} \operatorname{Hom}(C, Y) \rightarrow \mathcal{S} \operatorname{Hom}\left(C^{\prime}, Y\right)$, given by $U \mapsto e U$, where $U$ is a submodule of $\operatorname{Hom}(C, Y)$.

Remark 3 Both objects ${ }^{C}[\rightarrow Y\rangle$ and $\mathcal{S} \operatorname{Hom}(C, Y)$ related by the Auslander bijection $\eta_{C Y}$ concern morphisms ending in $Y$. Of course, in Proposition 3.9 we have seen already that all the indecomposable direct summands $C^{\prime}$ of the minimal right determiner $C(f)$ of a map $f: X \rightarrow Y$ satisfy $\operatorname{Hom}\left(C^{\prime}, Y\right) \neq 0$.

Looking at ${ }^{C}[\rightarrow Y\rangle$, we deal with morphisms ending in $Y$ and which are right $C$-determined. Looking at $\operatorname{Hom}(C, Y)$, we deal with maps ending in $Y$ and starting in $C$. One should be aware that a right minimal map ending in $Y$ and right $C$-determined usually will not start at $C$, thus the relationship between the elements of ${ }^{C}[\rightarrow Y\rangle$ and the submodules of $\operatorname{Hom}(C, Y)$ is really of interest! Note however that in case we deal with a map $f: C \rightarrow Y$ which is right $C$-determined (and starts in $C$ ), then

$$
\eta_{C Y}(f)=f \operatorname{Hom}(C, C)
$$

is just the $\Gamma(C)$-submodule of $\operatorname{Hom}(C, Y)$ generated by $f$.

We use the next two sections in order to transfer well-known properties of the lattice of submodules of a finite length module to the right $C$-factorization lattices, in particular the Jordan-Hölder theorem. In Sect. 5, we introduce the right $C$-length of a right $C$ determined map $f$ ending in $Y$, it corresponds to the the length of the factor module $\operatorname{Hom}(C, Y) / \eta_{C Y}(f)$. In Sect. 6 we will define the $C$-type of $f$ as the dimension vector of $\operatorname{Hom}(C, Y) / \eta_{C Y}(f)$.

\section{Right $C$-factorizations and right $C$-length}

The Auslander bijection asserts that the lattice ${ }^{C}[\rightarrow Y\rangle$ is a modular lattice of finite height, thus there is a Jordan-Hölder Theorem for ${ }^{C}[\rightarrow Y\rangle$; it can be obtained from the corresponding Jordan-Hölder Theorem for the submodule lattice $\mathcal{S} \operatorname{Hom}(C, Y)$. In Sects. 5 and 6, we are going to formulate the assertions for ${ }^{C}[\rightarrow Y\rangle$ explicitly. Here we consider composition series of submodules and factor modules of ${ }^{C}[\rightarrow Y\rangle$.

Let $h_{i}: X_{i} \rightarrow X_{i-1}$ be maps, where $1 \leq i \leq t$, with composition $f=h_{1} \ldots h_{t}$. The sequence $\left(h_{1}, h_{2}, \ldots, h_{t}\right)$ is called a right $C$-factorization of f of length $t$ provided 
the maps $h_{i}$ are non-invertible and the compositions $f_{i}=h_{1} \cdots h_{i}$ are right minimal and right $C$-determined, for $1 \leq i \leq t$. It sometimes may be helpful to deal also with right $C$-factorizations of length 0 ; by definition these are just the identity maps (or, if you prefer, the isomorphisms).

If $\left(h_{1}, \ldots, h_{t}\right)$ is a right $C$-factorization of a map $f$, then any integer sequence $0=i(0)<i(1)<\cdots<i(s)=t$ defines a sequence of maps $\left(h_{1}^{\prime}, h_{2}^{\prime}, \ldots, h_{s}^{\prime}\right)$ with $h_{j}^{\prime}=h_{i(j-1)+1} \cdots h_{i(j)}$ for $1 \leq j \leq s$. We use the following lemma inductively, in order to show that $\left(h_{1}^{\prime}, h_{2}^{\prime}, \ldots, h_{s}^{\prime}\right)$ is again a right $C$-factorization of $f$ and we say that $\left(h_{1}, h_{2}, \ldots, h_{t}\right)$ is a refinement of $\left(h_{1}^{\prime}, h_{2}^{\prime}, \ldots, h_{s}^{\prime}\right)$. In particular, any right $C$-factorization $\left(h_{1}, \ldots, h_{t}\right)$ of $f$ is a refinement of $f$.

Lemma 5.1 If $\left(h_{1}, \ldots, h_{t}\right)$ is a right $C$-factorization of length $t \geq 2$,then

$$
\left(h_{1}, \ldots, h_{i-1}, h_{i} h_{i+1}, h_{i+2}, \ldots, h_{t}\right)
$$

is a right $C$-factorization (of length $t-1$ ).

Proof We only have to check that $h_{i} h_{i+1}$ cannot be invertible. Assume $h_{i} h_{i+1}$ is invertible. Then $h_{i}$ is a split epimorphism. Since $f_{i}=h_{1} \cdots h_{i}$ is right minimal, it follows that $h_{i}$ is invertible, a contradiction.

We say that a right $C$-factorization $\left(h_{1}, h_{2}, \ldots, h_{t}\right)$ is maximal provided it does not have a refinement of length $t+1$.

Proposition 5.2 If $\left(h_{1}, \ldots, h_{t}\right)$ is a right $C$-factorization of a map $f$, and $f_{i}=$ $h_{1} \cdots h_{i}$ for $0 \leq i \leq t$, then

$$
\eta_{C Y}\left(f_{t}\right) \subset \cdots \subset \eta_{C Y}\left(f_{1}\right) \subset \eta_{C Y}\left(f_{0}\right)=\operatorname{Hom}(C, Y)
$$

is a chain of proper inclusions of submodules and any such chain is obtained in this way. The refinement of right $C$-factorizations corresponds to the refinement of submodule chains.

Proof This is a direct consequence of Auslander's Second Theorem.

Corollary 5.3 Any right $C$-factorization $\left(h_{1}, \ldots, h_{t}\right)$ has a refinement which is a maximal right $C$-factorization and all maximal right $C$-factorizations of $\left(h_{1}, \ldots, h_{t}\right)$ have the same length.

Proof This follows from Proposition 5.2 and the Jordan-Hölder theorem.

In particular, any right minimal right $C$-determined map $f$ has a refinement which is a maximal right $C$-factorization, say $\left(h_{1}, \ldots, h_{t}\right)$ and its length $t$ will be called the right $C$-length of $f$, we write $|f|_{C}$ for the right $C$-length of $f$. There is the following formula:

Proposition 5.4 Let $f: X \rightarrow Y$ be right minimal and right $C$-determined. Then 


$$
|f|_{C}=|\operatorname{Hom}(C, Y)|-\left|\eta_{C Y}(f)\right|
$$

where $|\operatorname{Hom}(C, Y)|$ denotes the length of the $\Gamma(C)$-module $\operatorname{Hom}(C, Y)$ and $\left|\eta_{C Y}(f)\right|$ the length of its $\Gamma(C)$-submodule $\eta_{C Y}(f)$.

The right equivalence class $\eta_{C Y}^{-1}(0)$. As we have mentioned in Sect. 4, it is of interest to determine the maps in the right equivalence class $\eta_{C Y}^{-1}(0)$.

Proposition 5.5 Let $C, Y$ be modules. Up to right equivalence, there is a unique right $C$-determined map $f$ ending in $Y$ with $|f|_{C}$ maximal. The submodule $\eta_{C Y}(f)$ of $\operatorname{Hom}(C, Y)$ is the zero module. If $f^{\prime}$ is any right $C$-determined map ending in $Y$, then $f=f^{\prime} h$ for some $h$.

Proof The lattice ${ }^{C}[\rightarrow Y\rangle$ has a unique zero element, namely $\eta_{C Y}^{-1}(0)$. Let $\eta_{C Y}^{-1}(0)=$ $[f\rangle$ for some right minimal map $f$. Then, the right $C$-length of $f$ has to be maximal and $[f\rangle \leq\left[f^{\prime}\right\rangle$ for any right $C$-determined map $f^{\prime}$ ending in $Y$.

In general it seems to be quite difficult to describe the maps $f$ such that $[f\rangle=$ $\eta_{C Y}^{-1}(0)$. But one should be aware that such a map $f$ always does exist: any pair $C, Y$ of $\Lambda$-modules determines uniquely up to right equivalence a map $f$ ending in $Y$, namely the right minimal, right $C$-determined map $f$ with $\eta_{C Y}(f)=0$.

Proposition 5.6 Let $C, Y$ be modules. The set $\eta_{C Y}^{-1}(0)$ is the right equivalence class of the zero map $0 \rightarrow Y$ if and only if $P(\operatorname{soc} Y)$ belongs to add $C$.

Proof This is a direct consequence of Corollary 3.7.

The special case of $C$ being projective. For an arbitrary projective module $C$, there is the following description of the right $C$-length of a right minimal, right $C$-determined morphism $f$. Here, we denote by $[M: S]$ the Jordan-Hölder multiplicity of the simple module $S$ in the module $M$, this is the number of factors in a composition series of $M$ which are isomorphic to $S$.

Proposition 5.7 Let $C$ be projective. The right minimal, right $C$-determined maps $f: X \rightarrow Y$ are up to right equivalence just the inclusion maps of submodules $X$ of $Y$ such that the socle of $Y / X$ is generated by $C$.

If $f: X \rightarrow Y$ is right minimal and right $C$-determined, then $f$ is injective and

$$
|f|_{C}=\sum_{P(S) \mid C}[\operatorname{Cok}(f): S] \text {. }
$$

The minimal element $\eta_{C Y}^{-1}(0)$ of ${ }^{C}[\rightarrow Y\rangle$ is the inclusion map $X \rightarrow Y$, where $X$ is the intersection of the kernels of all maps $Y \rightarrow Q(S)$, where $S$ is a simple module with $P(S)$ a direct summand of $C$. 
Proof Let $\mathcal{Q}$ be the set of modules $Q(S)$, where $S$ is a simple module with $P(S)$ a direct summand of $C$. Let $X$ be the intersection of the kernels of all maps $Y \rightarrow Q$ with $Q \in \mathcal{Q}$. Since $Y$ is of finite length, there are finitely many maps $g_{i}: Y \rightarrow Q\left(S_{i}\right)$ with $Q\left(S_{i}\right) \in \mathcal{Q}$, say $1 \leq i \leq m$, such that $X=\bigcap_{i=1}^{m} \operatorname{Ker}\left(g_{i}\right)$. Then $Y / X$ embeds into $\bigoplus_{i=1}^{m} Q\left(S_{i}\right)$, thus its socle is generated by $C$. It follows that the inclusion map $X \rightarrow Y$ is right $C$-determined. On the other hand, if $X^{\prime} \rightarrow Y$ is right minimal and right $C$-determined, then it is a monomorphism, thus we can assume that it is an inclusion map. In addition, we know that the socle of $Y / X^{\prime}$ is generated by $C$, thus $Y / X^{\prime}$ embeds into a finite direct sum of modules in $\mathcal{Q}$. It follows that $X^{\prime}$ is the intersection of some maps $Y \rightarrow Q$, where $Q \in \mathcal{Q}$, thus $X \subseteq X^{\prime}$.

There is the following consequence: The ${ }_{\Lambda} \Lambda$-length of any inclusion map $X \rightarrow Y$ (such a map is obviously right minimal and right $\Lambda$-determined) $i$ s precisely the length of $Y / X$.

For further results concerning the right $C$-length of maps, see Sect. 9.

\section{The right $C$-type of a right $C$-determined map}

Recall that we consider $\operatorname{Hom}(C, Y)$ as a $\Gamma(C)$-module, where $\Gamma(C)=\operatorname{End}(C)^{\mathrm{op}}$. The indecomposable projective $\Gamma(C)$-modules are of the form $\operatorname{Hom}\left(C, C_{i}\right)$, where $C_{i}$ is an indecomposable direct summand of $C$, thus the simple $\Gamma(C)$-modules are of the form $S\left(C_{0}\right)=$ top $\operatorname{Hom}\left(C, C_{0}\right)$.

Given an artin algebra $\Gamma$, we denote by $K_{0}(\Gamma)$ its Grothendieck group (of all $\Gamma$-modules modulo all exact sequences), it is the free abelian group with basis the set of isomorphism classes $[S]$ of the simple $\Gamma$-modules $S$. Given a $\Gamma$-module $M$, we denote by $\operatorname{dim} M$ the corresponding element in $K_{0}(\Gamma)$, called the dimension vector of $M$. Of course, $\operatorname{dim} M$ can be written as an integral linear combination $\operatorname{dim} M=$ $\sum_{[S]}[M: S][S]$, where the coefficient of $[S]$ is just the Jordan-Hölder multiplicity $[M: S]$ of $S$ in $M$. The elements of $K_{0}(\Gamma)$ with non-negative coefficients will be said to be the $\Gamma$-dimension vectors. If $\mathbf{e}$ is a $\Gamma$-dimension vector and $M$ is a $\Gamma$-module, we denote by $\mathcal{S}_{\mathrm{e}} M$ the subset of $\mathcal{S} M$ consisting of all submodules of $M$ with dimension vector e.

Let us return to the artin algebra $\Gamma(C)$, where $C$ is a $\Lambda$-module. Thhe Grothendieck group $K_{0}(\Gamma(C))$ is the free abelian group with basis the set of modules $S\left(C_{i}\right)$, where $C_{i}$ runs through a set of representatives of the isomorphism classes of the indecomposable direct summands $C_{i}$ of $C$. We are interested here in the dimension vectors of $\operatorname{Hom}(C, Y)$ and of its factor modules. Actually, we want to attach to each right $C$-determined map ending in $Y$ its right $C$-type type $_{C}(f)$ so that type $_{C}(f)=\operatorname{dim} \operatorname{Hom}(C, Y) / \eta_{C Y}(f)$. We start with pairs of neighbors in the right $C$-factorization lattice ${ }^{C}[\rightarrow Y\rangle$, since they correspond under $\eta_{C Y}$ to the composition factors of $\operatorname{Hom}(C, Y)$.

Let $f=h f^{\prime}$ and $f^{\prime}$ be right minimal, right $C$-determined maps. We say that the pair $\left(f, f^{\prime}\right)$ is a pair of $C$-neighbors provided $|f|_{C}=\left|f^{\prime}\right|_{C}+1$. Note that the pair $\left(f, f^{\prime}\right)$ in ${ }^{C}[\rightarrow Y\rangle$ is a pair of $C$-neighbors provided $[f\rangle<\left[f^{\prime}\right\rangle$ and there is no $f^{\prime \prime}$ with $[f\rangle<\left[f^{\prime \prime}\right\rangle<\left[f^{\prime}\right\rangle$ (of course, it is the condition $[f\rangle<\left[f^{\prime}\right\rangle$ which implies that there is a map $h$ with $\left.f=f^{\prime} h\right)$. 
Remark Let us consider a composition $f=f^{\prime} h$, where $f^{\prime}$ both are right minimal and right $C$-determined. It can happen that $h$ is also right minimal and right $C$-determined, but $f=f^{\prime} h$ is not right $C$-determined. Also it can happen that both maps $f^{\prime}$ and $f=$ $f^{\prime} h$ are right minimal and right $C$-determined, whereas $h$ is not right $C$-determined. Here are corresponding examples.

Example 5 We consider the path algebra $\Lambda$ of the linearly directed quiver $\Delta$ of type $\mathbb{A}_{3}$

$$
a \stackrel{\alpha}{\longrightarrow} b \stackrel{\beta}{\longrightarrow} c .
$$

Let $X=Q(a), X^{\prime}=Q(b), Y=Q(c)=S(c)$. There are non-zero maps

$$
Q(a) \stackrel{h}{\longrightarrow} Q(b) \stackrel{f^{\prime}}{\longrightarrow} S(c),
$$

and we let $f=f^{\prime} h$. All three maps $f^{\prime}, h, f$ are surjective and right minimal. The kernel of $f^{\prime}$ is the simple module $S(b)$, the kernel of $h$ is the simple module $S(a)$ and the kernel of $f$ is $P(b)$.

First, let $C=S(b) \oplus S(c)$, thus $\tau C=S(a) \oplus S(b)$ and both $f^{\prime}$ and $h$ are right $C$-determined, whereas $f$ is not right $C$-determined.

Second, let $C=Q(b) \oplus S(c)$, thus $\tau C=P(b) \oplus S(b)$. Then both $f$ and $f^{\prime}$ are right $C$-determined, whereas $h$ is not right $C$-determined.

Let $f: X \rightarrow Y$ and $f^{\prime}: X^{\prime} \rightarrow Y$ such that $\left(f, f^{\prime}\right)$ is a pair of neighbors. We say that $\left(f, f^{\prime}\right)$ is of type $C_{0}$ (or better of type $\left[S\left(C_{0}\right)\right]$ ) where $C_{0}$ is an indecomposable direct summand of $C$, provided there is a map $\phi: C_{0} \rightarrow X^{\prime}$ such that $f^{\prime} \phi$ does not factor through $f$. Such a summand $C_{0}$ must exist, since otherwise $f^{\prime}$ would factor through $f$, due to the fact that $f$ is right $C_{0}$-determined. The following proposition shows that $C_{0}$ is uniquely determined.

Proposition 6.1 If $\left(f, f^{\prime}\right)$ is a pair of $C$-neighbors of type $C_{0}$, then $\eta_{C Y}\left(f^{\prime}\right) / \eta_{C Y}(f)$ is isomorphic to the simple $\Gamma(C)$-module $S\left(C_{0}\right)=\operatorname{top} \operatorname{Hom}\left(C, C_{0}\right)$. Thus, the type of a pair of $C$-neighbors is well-defined.

Thus, if $\left(f, f^{\prime}\right)$ is a pair of $C$-neighbors of type $C_{0}$, we may write $\operatorname{type}_{C}\left(f, f^{\prime}\right)=$ $\left[S\left(C_{0}\right)\right] \in K_{0}(\Gamma(C))$.

Proof Let $\phi: C_{0} \rightarrow X^{\prime}$ be a map such that $f^{\prime} \phi$ does not factor through $f$. We obtain a homomorphism of $\Gamma(C)$-modules

$$
\operatorname{Hom}\left(C, f^{\prime} \phi\right): \operatorname{Hom}\left(C, C_{0}\right) \rightarrow \operatorname{Hom}(C, Y)
$$

which maps into $f^{\prime} \operatorname{Hom}\left(C, X^{\prime}\right)$ (since the image consists of the maps $f^{\prime} \phi \psi$ with $\left.\psi: C \rightarrow C_{0}\right)$, We claim that $\operatorname{Hom}\left(C, f^{\prime} \phi\right)$ does not map into $f \operatorname{Hom}(C, X)$. Assume, for the contrary, that $\operatorname{Hom}\left(C, f^{\prime} \phi\right)$ maps into $f \operatorname{Hom}(C, X)$. Choose $m: C_{0} \rightarrow C$ and $e: C \rightarrow C_{0}$ with $e m=1$. By assumption, the element $\operatorname{Hom}\left(C, f^{\prime} \phi\right)(e)=f^{\prime} \phi e$ belongs to $f \operatorname{Hom}(C, X)$, thus there is $\phi^{\prime}: C \rightarrow X$ with $f^{\prime} \phi e=f \phi^{\prime}$ and therefore

$$
f^{\prime} \phi=f^{\prime} \phi e m=f \phi^{\prime} m
$$


shows that $f^{\prime} \phi$ factor through $f$, a contradiction.

Thus, the image of $\operatorname{Hom}\left(C, f^{\prime} \phi\right)$ is a $\Gamma(C)$-submodule of $\operatorname{Im} \operatorname{Hom}\left(C, f^{\prime}\right)$ which is not contained in $\operatorname{Im}\left(C, f^{\prime}\right)$ and which is an epimorphic image of the projective module $\operatorname{Hom}\left(C, C_{0}\right)$. Since we know that $\operatorname{Im} \operatorname{Hom}(C, f)$ is a maximal submodule of $\operatorname{Im} \operatorname{Hom}\left(C, f^{\prime}\right)$, it follows that

$\operatorname{Im} \operatorname{Hom}\left(C, f^{\prime}\right) / \operatorname{Im} \operatorname{Hom}\left(C, f^{\prime}\right) \simeq \operatorname{top} \operatorname{Im} \operatorname{Hom}\left(C, f^{\prime} \phi\right) \simeq \operatorname{top} \operatorname{Hom}\left(C, C_{0}\right)$

This is what we wanted to show.

We note the following: If $\left(f, f^{\prime}\right)$ is a pair of $C$-neighbors and $f=f^{\prime} h$, then $h$ may be neither injective nor surjective. Let us exhibit examples with $f^{\prime}=1_{Y}$.

Example 6 As in the examples 5 , let $\Delta$ be the linearly directed quiver of type $\mathbb{A}_{3}$ and take now as $\Lambda$ the path algebra of $\Delta$ modulo the zero relation $\alpha \beta$.

Let $Y=P(c)$ and $C=S(b)$, then

$$
{ }^{S(b)}[\rightarrow P(c)\rangle \quad \leftrightarrow \quad \mathcal{S} \operatorname{Hom}(S(b), P(c))
$$

are lattices with precisely two elements: in ${ }^{S(2)}[\rightarrow P(3)\rangle$, there is the right equivalence class of the identity map $f^{\prime}=1_{P(c)}$ as well as the right equivalence class of any nonzero map $f: P(b) \rightarrow P(c)$. Note that $f$ is right minimal and right $S(b)$-determined, and it is neither mono nor epi.

Now consider a right minimal right $C$-determined map $f$ ending in $Y$. As we have mentioned, we want to attach to $f$ an element $\operatorname{type}_{C}(f) \in K_{0}(\Gamma(C))$.

Proposition 6.2 Let $C, Y$ be $\Lambda$-modules. Let $f$ be right minimal and right $C$-determined map ending in $Y$ with maximal right $C$-factorization $h_{1}, \ldots, h_{t}$. Write $f_{i}=$ $h_{1} \cdots h_{i}$, for $0 \leq i \leq t$. Then

$$
\text { type }_{C} f=\sum_{i=1}^{t} \operatorname{type}_{C}\left(f_{i}, f_{i-1}\right)
$$

is a well-defined element of $K_{0}(\Gamma(C))$ and we have

$$
\operatorname{type}_{C}(f)=\operatorname{dim} \operatorname{Hom}(C, Y) / \eta_{C Y}(f)=\mathbf{d}-\operatorname{dim} \eta_{C Y}(f),
$$

with $\mathbf{d}=\operatorname{dim} \operatorname{Hom}(C, Y)$.

Proof Unter the Auslander bijection $\eta_{C Y}$, the chain

$$
[f\rangle=\left[f_{t}\right\rangle<\left[f_{t-1}\right\rangle<\cdots<\left[f_{0}\right\rangle
$$

is mapped to a chain of submodules

$$
\eta_{C Y}(f)=\eta_{C Y}\left(f_{t}\right) \subset \eta_{C Y}\left(f_{t-1}\right) \subset \cdots \subset \eta_{C Y}\left(f_{0}\right)=\operatorname{Hom}(C, Y)
$$


with simple factors $\eta_{C Y}\left(f_{i-1}\right) / \eta_{C Y}\left(f_{i}\right)$, thus we obtain in this way a composition series of $\operatorname{Hom}(C, Y) / \eta_{C Y}(f)$. The Jordan-Hölder theorem for $\operatorname{Hom}(C, Y) / \eta_{C Y}(f)$ asserts that this yields the dimension vector $\operatorname{dim} \operatorname{Hom}(C, Y) / \eta_{C Y}(f)$, independent of the choice of the composition series.

For any $\Gamma(C)$-dimension vector $\mathbf{e}$, let us denote by ${ }^{C}[\rightarrow Y\rangle^{\mathbf{e}}$ the set of all elements $[f\rangle$ in ${ }^{C}[\rightarrow Y\rangle$ such that type ${ }_{C}(f)=\mathbf{e}$. Note that ${ }^{C}[\rightarrow Y\rangle^{\mathbf{e}}$ is non-empty only in case $\mathbf{e} \leq \operatorname{dim} \operatorname{Hom}(C, Y)$, thus we obtain a decomposition

$$
{ }^{C}[\rightarrow Y\rangle=\bigsqcup_{\mathbf{e}}{ }^{C}[\rightarrow Y\rangle^{\mathbf{e}}
$$

into a finite number of disjoint subsets.

Proposition 6.3 The Auslander bijection $\eta_{C Y}$ yields a bijection

$$
{ }^{C}[\rightarrow Y\rangle^{\mathbf{e}} \stackrel{1: 1}{\longrightarrow} \mathcal{S}_{\mathbf{d}-\mathbf{e}} \operatorname{Hom}(C, Y)
$$

for every $\Gamma(C)$-dimension vector $\mathbf{e}$.

Assume now that $k$ is an algebraically closed field and that $\Lambda$ and $\Gamma$ are $k$-algebra. If $M$ is a $\Gamma$-module and $\mathbf{e}$ a dimension vector for $\Gamma$, we write $\mathbb{G}_{\mathbf{e}} M$ instead of $\mathcal{S}_{\mathbf{e}} M$. Note that $\mathbb{G}_{\mathbf{e}} M$ is in a natural way an algebraic variety, it is called a quiver Grassmannian. Namely, all the $\Gamma$-modules with dimension vector e have the same $k$-dimension, say $e$ (if $\mathbf{e}=\sum_{[S]} e_{S}[S]$, then $e=\sum_{[S]} e_{S} \operatorname{dim}_{k} S$ ). Denote by $\mathbb{G}_{e}\left({ }_{k} M\right)$ the usual Grassmannian of all $e$-dimensional subspaces $U$ of the vector space ${ }_{k} M$. Using Plücker coordinates one knowns that $\mathbb{G}_{e}\left({ }_{k} M\right)$ is a closed subset of a projective space, thus $\mathbb{G}_{e}\left({ }_{k} M\right)$ is a projective variety. Now $\mathbb{G}_{\mathbf{e}} M$ is a subset of $\mathbb{G}_{e}\left({ }_{k} M\right)$ defined by the vanishing of some polynomials (which express the fact that we consider submodules $U$ with a fixed dimension vector), thus also $\mathbb{G}_{\mathbf{e}} M$ is an algebraic variety and indeed a projective variety (but usually not even connected).

Proposition 6.3 can be reformulated as follows:

Proposition 6.4 The Auslander bijection $\eta_{C Y}$ yields a bijection

$$
{ }^{C}[\rightarrow Y\rangle^{\mathbf{e}} \stackrel{1: 1}{\longrightarrow} \mathbb{G}_{\mathbf{d}-\mathbf{e}} \operatorname{Hom}(C, Y)
$$

for every $\Gamma(C)$-dimension vector $\mathbf{e}$.

In particular, we see that the set ${ }^{C}[\rightarrow Y\rangle^{\mathbf{e}}$ is a projective variety: these Auslander varieties (as they should be called) furnish an important tool for studying the right equivalence classes of maps ending in a given module. As we have mentioned at the end of Sect. 2, the study of the set of right minimal maps ending in a fixed module $Y$ can be separated nicely into that of the local symmetries described by the right automorphism 
groups and that of the global directedness given by the right factorization lattice. Auslander's first theorem describes the right factorization lattice as the filtered union of the right $C$-factorization lattices, and, as we now see, these right $C$-factorization lattices are finite disjoint unions of (transversal) subsets which are projective varieties, the Auslander varieties.

Remark It seems that quiver Grassmannians first have been studied by Schofield [42] and Crawley-Boevey [10] in order to deal with generic properties of quiver representations. In 2006, Caldero and Chapoton [9] observed that quiver Grassmannians can be used effectively in order to analyse the structure of cluster algebras as introduced by Fomin and Zelevinsky. Namely, it turns out that cluster variables can be described using the Euler characteristic of quiver Grassmannians. In this way quiver Grassmannians are now an indispensable tool for studying cluster algebras and quantum cluster algebras. We should add that quiver Grassmannians were also used (at least implicitly) in the study of quantum groups, see for example the calculation of Hall polynomials in [34]. A large number of papers is presently devoted to special properties of quiver Grassmannians.

There is the famous assertion that any projective variety is a quiver Grassmannian, see the paper [29] by Reineke (answering in this way a question by Keller) as well as blogs by Le Brujn [26] (with a contribution by Van den Bergh) and by Baez [6]. Actually, the construction as proposed by Van den Bergh in Le Bruyn's blog is much older, it has been mentioned explicitly already in 1996 by Hille [21] dealing with moduli spaces of thin representations (see the example at the end of that paper), and it can be traced back to earlier considerations of Huisgen-Zimmermann dealing with moduli spaces of serial modules, even if they were published only later (see [22], Theorem G, but also [8], Corollary B, and [12], Example 5.4). It follows from Proposition 4.6 above that given a strictly wild algebra $\Lambda$ and any projective variety $V$, there are $\Lambda$-modules $C, Y$ and a dimension vector $\mathbf{e}$ such that ${ }^{C}[\rightarrow Y\rangle{ }^{\mathbf{e}}$ is isomorphic to $V$. We will show in [39] that this holds true for all controlled wild algebras.

\section{Maps of right $C$-length 1}

By definition, a right minimal right $C$-determined map $f$ has right $C$-length 1 provided $f$ is not invertible and given any factorization $f=f^{\prime} h$ with $f^{\prime}$ right minimal right $C$-determined, then one of the maps $f^{\prime}, h$ is invertible. Let us denote by ${ }^{C}[\rightarrow Y\rangle^{1}$ the set of right equivalence classes of the maps ending in $Y$ which have right $C$-length 1 .

Warning: an irreducible map $f$ is of course right minimal, but if $f$ is irreducible and right $C$-determined, we may have $|f|_{C}>1$. For example, consider the Kronecker quiver, take $C={ }_{\Lambda} \Lambda$. The irreducible map $f: P_{0} \rightarrow P_{1}$ has $|f|_{C}=2$ (note the factorizazion $\left.P_{0} \subset \operatorname{rad} P_{1} \subset P_{1}\right)$.

Here is an immediate consequence of Proposition 5.4.

Corollary 7.1 Let $f: X \rightarrow Y$ be right minimal and right $C$-determined. Then $|f|_{C}=1$ if and only if $\eta_{C Y}(f)$ is a maximal $\Gamma(C)$-submodule of $\operatorname{Hom}(C, Y)$.

If we denote by $\mathcal{S}_{m} \operatorname{Hom}(C, Y)$ the set of maximal submodules of $\operatorname{Hom}(C, Y)$, then the restriction of $\eta_{C Y}$ furnishes a bijection 


$$
{ }^{C}[\rightarrow Y\rangle^{1} \stackrel{1: 1}{\longrightarrow} \mathcal{S}_{m} \operatorname{Hom}(C, Y)
$$

In order to analyze maps of right $C$-length 1 , we will need the following lemma.

Lemma 7.2 Assume that $f: X \rightarrow Y$ and $f^{\prime}: X^{\prime} \rightarrow Y$ are epimorphisms with $f=f^{\prime} h$. Then we have the following commutative diagram with exact rows:

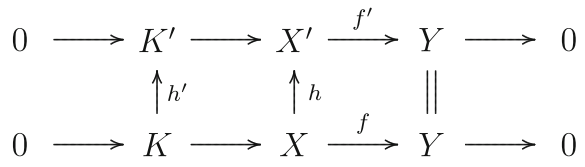

If $f$ is right minimal and $h^{\prime}$ is a split epimorphism, then also $f^{\prime}$ is right minimal.

Remark Observe that it is not enough to assume that $h^{\prime}$ is an epimorphism. As an example, take the indecomposable injective Kronecker module $X=Q_{1}$ of length 3, let $K$ be a submodule of length 2 , and $K^{\prime}=K / \operatorname{soc}$. Then $Q_{1} \rightarrow Q_{1} / K$ is right minimal. But the induced sequence is just the short exact sequence $K / \operatorname{soc} \rightarrow Q_{1} / \operatorname{soc} \rightarrow Y$ which splits.

Proof Denote the kernel of $h^{\prime}$ by $K^{\prime \prime}$, thus we can assume that $K=K^{\prime} \oplus K^{\prime \prime}$ such that $h^{\prime}$ is the canonical projection $K \rightarrow K^{\prime}$ with kernel $K^{\prime \prime}$. Assume that $X^{\prime}=U \oplus V$, where $U$ is contained in the kernel of $f^{\prime}$, thus $U \subseteq K^{\prime}$. Since $X^{\prime}=X / K^{\prime \prime}$, there are submodules $U^{\prime}, V^{\prime}$ of $X^{\prime}$ both containing $K^{\prime \prime}$ such that $U^{\prime}+V^{\prime}=X^{\prime}$ and $U^{\prime} \cap V^{\prime}=$ $K^{\prime \prime}$, with $U=U^{\prime} / K^{\prime \prime}$ and $V=V^{\prime} / K^{\prime \prime}$.

Consider $U^{\prime \prime}=U^{\prime} \cap K^{\prime}$, this is a submodule of the kernel $K$ of $f$. Also, $U^{\prime}=$ $K^{\prime \prime}+U^{\prime \prime}$ (using the modular law). Thus we have $U^{\prime \prime} \cap V^{\prime}=U^{\prime} \cap K^{\prime} \cap V^{\prime} \subseteq K^{\prime} \cap K^{\prime \prime}=0$ and $U^{\prime \prime}+V^{\prime}=U^{\prime \prime}+V^{\prime}+K^{\prime \prime}=U^{\prime}+V^{\prime}=X$. This shows that $U^{\prime \prime}$ is a direct summand of $X$ which is contained in the kernel of $f$. Since $f$ is right minimal, we see that $U^{\prime \prime}=0$. Since $U^{\prime}=K^{\prime \prime}+U^{\prime \prime}=K^{\prime \prime}$, it follows that $U=0$.

Corollary 7.3 Let $C$ be a module. Let $f: X \rightarrow Y$ be a right minimal right $C$ determined epimorphism with $|f|_{C}=1$. Then the kernel of $f$ is indecomposable.

Proof Let $K=\tau C$. The kernel of $f$ has to be non-zero, thus assume it is decomposable, say equal to $K_{1} \oplus K_{2}$ with non-zero modules $K_{1}, K_{2} \in$ add $K$. The canonical projection $p_{1}: K_{1} \oplus K_{2} \rightarrow K_{1}$ yields a commutative diagram with exact rows:

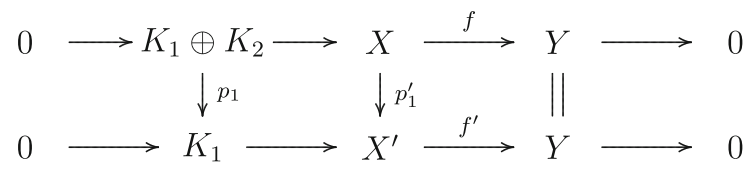

Since $f$ is right minimal and $p_{1}$ is a split epimorphism, lemma 7.2 asserts that $f^{\prime}$ is right minimal. Since $f^{\prime}$ is also right $C$-determined, we see that $|f|_{C} \geq 2$, a contradiction. 
Proposition 7.4 Let $C$ be indecomposable and non-projective and let $K=\tau C$. If

$$
\epsilon: \quad 0 \longrightarrow K^{t} \longrightarrow X \stackrel{f}{\longrightarrow} Y \longrightarrow 0
$$

is an exact sequence, then $[f\rangle$ belongs to ${ }^{C}[\rightarrow Y\rangle^{1}$ if and only if $t=1$ and the equivalence class $[\epsilon]$ is a non-zero element of the $\Gamma(K)$-socle of $\operatorname{Ext}^{1}(Y, K)$.

Proof First, assume that $[f\rangle$ belongs to ${ }^{C}[\rightarrow Y\rangle{ }^{1}$. According to Corollary 7.3, we must have $t=1$. Also, since $f$ is not split epi, we see that $[\epsilon]$ is a non-zero element of $\operatorname{Ext}^{1}(Y, K)$. Assume that $[\epsilon]$ does not belong to the $\Gamma(K)$-socle of $\operatorname{Ext}^{1}(Y, K)$. Then there is a nilpotent endomorphism $\phi$ of $K$ such that the induced exact sequence does not split. Thus there is a commutative diagram with exact rows

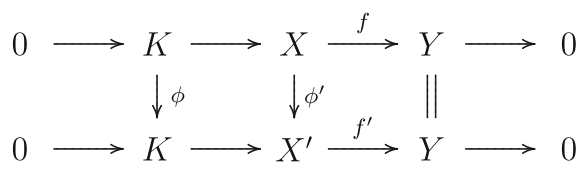

with the upper row being the sequence $\epsilon$. Since the lower sequence does not split, the map $f^{\prime}$ is right minimal. The kernel shows that $f^{\prime}$ is also $C$-determined. Since $\phi^{\prime}$ is not invertible, the factorization $f=f^{\prime} \phi^{\prime}$ shows that $\left(\phi^{\prime}, f^{\prime}\right)$ is a $C$-factorization of $f$ of length at least 2 , thus $|f|_{C} \geq 2$, a contradiction. This shows that $[\epsilon]$ belongs to the $\Gamma(K)$-socle of $\operatorname{Ext}^{1}(Y, K)$.

Conversely, assume that $t=1$ and $[\epsilon]$ is a non-zero element of the $\Gamma(K)$-socle of $\operatorname{Ext}^{1}(Y, K)$. Now $f$ is right minimal, right $C$-determined, and not an isomorphism, thus $|f|_{C} \geq 1$. Assume that $|f|_{C} \geq 2$, thus there is a $C$-factorization $\left(h_{1}, h_{2}\right)$ of $f$. Since $f=h_{2} h_{1}$ is surjective, also $h_{2}$ is surjective. Since $h_{2}$ is right minimal, right $C$-determined and not invertible, its kernel has to be of the form $K^{t}$ for some $t \geq 1$. Thus there is a commutative diagram with exact rows of the following form

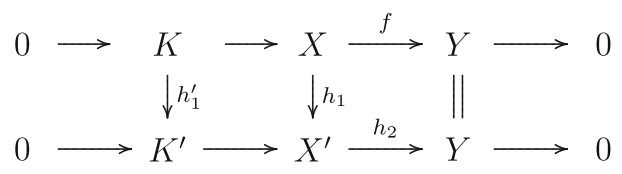

where again the upper row is $\epsilon$. Write $h_{1}^{\prime}=\left(\phi_{1}, \ldots, \phi_{t}\right)$ with endomorphisms $\phi_{i}$ : $K \rightarrow K$. If all $\phi_{i}$ belong to the radical of $\Gamma(K)$, then all the sequences induced from $\epsilon$ by the maps $\phi_{i}$ split, thus also the lower sequence splits, since it is induced from $\epsilon$ by $h_{1}^{\prime}$. Thus, at least one of the maps $\phi_{i}$ has to be invertible and therefore $h_{1}^{\prime}$ is a split monomorphism.

If $t>1$, then the lower sequence splits off a sequence $0 \rightarrow K \stackrel{1}{\rightarrow} K \rightarrow 0 \rightarrow 0$, but this means that $h_{2}$ is not right minimal. Thus $t=1$. But then $h_{1}^{\prime}$ is an automorphism, thus $h_{1}$ is invertible, a contradiction. This shows that $|f|_{C}=1$.

We say that an epimorphism $f$ is epi-irreducible, provided for any factorization $f=f^{\prime} f^{\prime \prime}$ with $f^{\prime \prime}$ a proper epimorphism, the map $f^{\prime}$ is a split epimorphism (the dual concept of mono-irreducible maps has been considered in [35]). 
Proposition 7.5 Let $C$ be indecomposable, non-projective and let $K=\tau C$. If $f$ : $X \rightarrow Y$ is an epi-irreducible epimorphism with kernel $K=\tau C$, then $f$ belongs to $C_{[\rightarrow Y\rangle^{1} \text {. }}$

Proof According to Proposition 7.4, we have to show that the given exact sequence

$$
\epsilon: \quad 0 \longrightarrow K \longrightarrow X \stackrel{f}{\longrightarrow} Y \longrightarrow 0
$$

belongs to the $\Gamma(K)$-socle of $\operatorname{Ext}^{1}(Y, K)$. Thus, let $\phi$ be a non-invertible endomorphism of $K$, write it in the form $\phi=\phi^{\prime} \phi^{\prime \prime}$ with $\phi^{\prime \prime}$ epi and $\phi^{\prime}$ mono. Consider the exact sequence induced from $\epsilon$ by $\phi^{\prime \prime}$

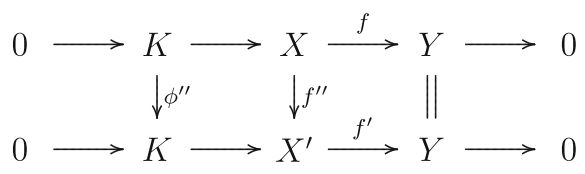

Since $\phi^{\prime \prime}$ is a proper epimorphism, also $f^{\prime \prime}$ is a proper epimorphism, thus $f^{\prime}$ is a split epimorphism. But this implies that also the exact sequence induced from $\epsilon$ by $\phi$ splits.

We will need some basic facts concerning the Gabriel-Roiter measure of finite length modules, see [35]. The Gabriel-Roiter measure of a module $M$ will be denoted by $\gamma(M)$. We recall that any indecomposable module $M$ which is not simple has a Gabriel-Roiter submodule $M^{\prime}$, this is a certain indecomposable submodule of $M$ and the embedding $M^{\prime} \rightarrow M$ is called a Gabriel-Roiter inclusion. Recall that a Gabriel-Roiter inclusion $M^{\prime} \rightarrow M$ is mono-irreducible: this means that for any proper submodule $M^{\prime \prime}$ of $M$ with $M^{\prime} \subseteq M^{\prime \prime}$, the inclusion $M^{\prime} \subseteq M^{\prime \prime}$ splits. As a consequence, given any nilpotent endomorphism $f$ of $M / M^{\prime}$, the sequence induced from $0 \rightarrow$ $M^{\prime} \rightarrow M \rightarrow M / M^{\prime} \rightarrow 0$ using $f$ splits. Also, it follows that the cokernel $M / M^{\prime}$ of a Gabriel-Roiter inclusion is indecomposable (and not projective).

Of course, we may use duality and consider a Gabriel-Roiter submodule $U$ of $D M$, the corresponding projection $M=D^{2} M \rightarrow D U$ will be called a co-GabrielRoiter projection. By duality, a co-Gabriel-Roiter projection is an epi-irreducible epimorphism with (non-injective) indecomposable kernel.

Corollary 7.6 Let $M$ be an indecomposable module which is not simple and let $f$ : $M \rightarrow Y$ be a co-Gabriel-Roiter projection, say with kernel $K$. Let $C=\tau^{-} K$. Then $f$ is right minimal, right $C$-determined and $|f|_{C}=1$, thus $[f\rangle$ belongs to ${ }^{C}[\rightarrow Y\rangle^{1}$.

Remark If $M$ is indecomposable and not simple, we also may consider a GabrielRoiter submodule $U$ of $M$, say with projection $p: M \rightarrow M / U=Y^{\prime}$ and consider $C^{\prime}=\tau^{-} U$. Then $p$ is right minimal and right $C^{\prime}$-determined, however in general there is not a fixed number $t$ such that $[p\rangle$ belongs to $C^{\prime}\left[\rightarrow Y^{\prime}\right\rangle^{t}$ or to $C^{\prime}\left[\rightarrow Y^{\prime}\right\rangle_{t}$. A typical example is example 8 presented in the next section. The two modules $P(b)$ and $\tau^{-} S(a)$ both have $P(a)$ as a Gabriel-Roiter submodule with factor module $S(b)$. Let $C^{\prime}=\tau^{-} P(a)$. The projection $P(b) \rightarrow S(b)$ belongs to $C^{\prime}[\rightarrow S(b)\rangle^{2}={ }^{C^{\prime}}[\rightarrow S(b)\rangle_{0}$ whereas the projection $\tau^{-} S(a) \rightarrow S(b)$ belongs to ${ }^{C^{\prime}}[\rightarrow S(b)\rangle^{1}={ }^{C^{\prime}}[\rightarrow S(b)\rangle_{1}$. 


\section{Epimorphisms in $[\rightarrow Y\rangle$}

The set of right equivalence classes $[f\rangle$ where $f$ is an epimorphism is obviously a coideal of the lattice $[\rightarrow Y\rangle$, we denote it by $[\rightarrow Y\rangle_{\text {epi }}$. Since the pullback of an epimorphism is again an epimorphism, we see that $[\rightarrow Y\rangle_{\text {epi }}$ is closed under meets. Also, for any module $C$, the subset ${ }^{C}[\rightarrow Y\rangle_{\text {epi }}$ of ${ }^{C}[\rightarrow Y\rangle$ consisting of the right equivalence classes of all right $C$-determined epimorphisms ending in $Y$ is a coideal which is closed under meets. Since ${ }^{C}[\rightarrow Y\rangle$ is a lattice of finite height, we see that ${ }^{C}[\rightarrow Y\rangle_{\text {epi }}$ has a unique minimal element, say $\left[f_{0}\right\rangle$ and our first aim will be to describe $\eta_{C Y}\left(f_{0}\right)$.

Before we deal with this question, let us point out in which way the projectivity or non-projectivity of indecomposable direct summands of $C$ are related to the fact that right minimal right $C$-determined morphisms $f$ are mono or epi. If $f$ is a monomorphism, then $f$ is right minimal and right $\Lambda$-determined (see Corollary 3.8), thus right $C$-determined for some projective module $C$. Conversely, if $C$ is projective, then any right minimal, right $C$-determined morphism is a monomorphism (see Corollary 3.7). Namely, if $K$ is an indecomposable direct summand of the kernel of $f$, where $f$ is right minimal, then $K$ is not injective and $\tau^{-} K$ is a direct summand of any module $C$ such that $f$ is right $C$-determined. Of course, since $K$ is not injective, $\tau^{-} K$ is an indecomposable non-projective module. One should be aware that a morphism may be right $C$-determined for some module $C$ without any indecomposable projective direct summand, without being surjective.

Example 7 As in example 6, we take as $\Lambda$ the path algebra of the linearly directed quiver $\Delta$ of type $\mathbb{A}_{3}$, modulo the zero relation $\alpha \beta$. Again, let $Y=P(c)$ and $C=S(b)$. As we have mentioned already, the non-zero maps $f: P(b) \rightarrow P(c)$ are not surjective, but right $S(b)$-determined, and, of course, $S(b)$ is not projective. (As we will see below, it is essential for this feature that the kernel of $f$ has injective dimension at least 2; for a general discussion of maps which are not surjective, but right $C$-determined by a module $C$ without any indecomposable projective direct summands, we refer to [38]).

The submodule $\operatorname{Hom}(C, \mathcal{P}, Y)$ of $\operatorname{Hom}(C, Y)$. We denote by $\operatorname{Hom}(C, \mathcal{P}, Y)$ the set of morphisms $C \rightarrow Y$ which factor through a projective module. Note that $\operatorname{Hom}(C, \mathcal{P}, Y)$ is a $\Gamma(C)$-submodule of $\operatorname{Hom}(C, Y)$.

Proposition 8.1 Assume that $f: X \rightarrow Y$ is right $C$-determined. Then $f$ is surjective if and only if $\eta_{C Y}(f) \supseteq \operatorname{Hom}(C, \mathcal{P}, Y)$.

Proof One direction is a trivial verification: First assume that $f$ is surjective. Let $h$ belong to $\operatorname{Hom}(C, \mathcal{P}, Y)$, thus $h=h_{2} h_{1}$ where $h_{1}: C \rightarrow P$ ) and $h_{2}: P \rightarrow Y$ with $P$ projective. Since $f$ is surjective and $P$ is projective, there is $h_{2}^{\prime}: P \rightarrow X$ such that $h_{2}=f h_{2}^{\prime}$. Thus shows that $h=h_{2} h_{1}=f h_{2}^{\prime} h_{1}$ belongs to $f \operatorname{Hom}(C, X)=\eta_{C Y}(f)$.

The converse is more interesting, here we have to use that $f$ is right $C$-determined. We assume that $\eta_{C Y}(f) \supseteq \operatorname{Hom}(C, \mathcal{P}, Y)$. Let $p: P(Y) \rightarrow Y$ be a projective cover of $Y$. Consider an arbitrary morphism $\phi: C \rightarrow P(Y)$. The composition $p \phi$ belongs to $\operatorname{Hom}(C, \mathcal{P}, Y)$, thus to $\eta_{C Y}(f)=f \operatorname{Hom}(C, X)$. Since $f$ is right $C$-determined, it follows that $p$ itself factors through $f$, say $p=f p^{\prime}$ for some $p^{\prime}: P(Y) \rightarrow X$. Now the composition $f p^{\prime}=p$ is surjective, there $f$ has to be surjective. 
Let us denote by ${ }^{C}[\rightarrow Y\rangle_{\text {epi }}$ the subset of ${ }^{C}[\rightarrow Y\rangle$ given by all elements $[f\rangle$ with $f$ an epimorphism.

Proposition 8.2 The restriction of the Auslander bijection $\eta_{C Y}$ yields a poset isomorphism

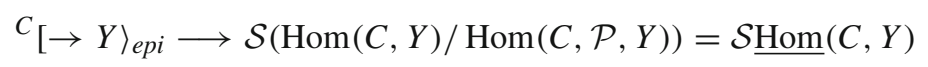

such that the following diagram commutes:

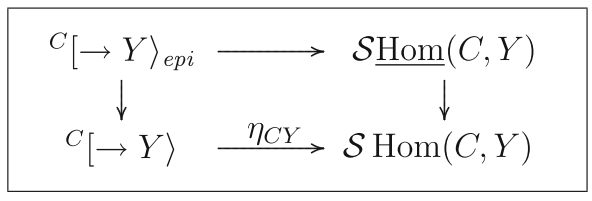

Here, the vertical maps are the canonical inclusions.

Proof It is well-known that given a module $M$ and a submodule $M^{\prime}$, then the lattice of submodules of the factor module $M / M^{\prime}$ is canonically isomorphic to the lattice of the submodules $U$ of $M$ satisfying $M^{\prime} \subseteq U$. This is the vertical map on the right.

More generally, dealing with a morphism $f$ which is right $C$-determined, we can recover the image of $f$ as follows:

Proposition 8.3 Let $f: X \rightarrow Y$ be right $C$-determined. Then one recovers the image of $f$ as the largest submodule $Y^{\prime}$ of $Y$ (with inclusion map $u: Y^{\prime} \rightarrow Y$ ) such that $u \operatorname{Hom}\left(C, \mathcal{P}, Y^{\prime}\right) \subseteq f \operatorname{Hom}(C, X)$.

Proof Let $Y^{\prime}$ be the image of $f$ with inclusion map $u$ and $u f^{\prime}=f$ (with $f^{\prime}$ surjective). First of all, we show that $u \operatorname{Hom}\left(C, \mathcal{P}, Y^{\prime}\right) \subseteq f \operatorname{Hom}(C, X)$. Let $\phi^{\prime}: C \rightarrow \Lambda$ and $\phi^{\prime \prime}: \Lambda \rightarrow Y^{\prime}$ (the maps $\phi^{\prime \prime} \phi^{\prime}$ obtained in this way generate $\operatorname{Hom}\left(C, \mathcal{P}, Y^{\prime}\right.$ ) additively). We want to show that $u \phi^{\prime \prime} \phi$ factors through $f$. Since $f^{\prime}: X \rightarrow Y^{\prime}$ is surjective, there is $\psi: \Lambda \rightarrow X$ such that $\phi^{\prime \prime}=f^{\prime} \psi$ (since $\Lambda$ is projective). Thus $u \phi^{\prime \prime} \phi^{\prime}=u f^{\prime} \psi \phi^{\prime}=f \psi \phi^{\prime}$. Thus $u \phi^{\prime \prime} \phi^{\prime}$ factors through $f$.

On the other hand, let $u^{\prime \prime}: Y^{\prime \prime} \rightarrow Y$ be a submodule of $Y$ such that $u^{\prime \prime} \operatorname{Hom}\left(C, \mathcal{P}, Y^{\prime \prime}\right) \subseteq f \operatorname{Hom}(C, X)$. Let $p: P\left(Y^{\prime \prime}\right) \rightarrow Y^{\prime \prime}$ be a projective cover. Consider the map $f^{\prime}=u^{\prime \prime} p: P\left(Y^{\prime \prime}\right) \rightarrow Y$. It has the property that for all maps $\phi: C \rightarrow P\left(Y^{\prime \prime}\right)$ the composition $f^{\prime} \phi$ factors through $f$ (namely $f^{\prime} \phi=u^{\prime \prime} p \phi$ belongs to $\left.u^{\prime \prime} \operatorname{Hom}\left(C, \mathcal{P}, Y^{\prime \prime}\right) \subseteq f \operatorname{Hom}(C, X)\right)$. But $f$ is right $C$-determined, thus we conclude that $f^{\prime}$ factors through $\alpha$, say $f^{\prime}=f \phi^{\prime}$ for some $\phi^{\prime}: C \rightarrow P\left(Y^{\prime \prime}\right)$. Thus the image $Y^{\prime \prime}$ of $f^{\prime}$ is contained in the image $Y^{\prime}$ of $f$. This is what we wanted to prove.

We recover in this way Proposition 8.1. Namely, if $f$ is surjective, then $Y$ is the image of $f$, thus $Y$ is one of the submodule $Y^{\prime}$ with $u \operatorname{Hom}\left(C, \mathcal{P}, Y^{\prime}\right) \subseteq f \operatorname{Hom}(C, X)$, thus $\operatorname{Hom}(C, \mathcal{P}, Y) \subseteq f \operatorname{Hom}(C, Y)$.

Conversely, if $\operatorname{Hom}(C, \mathcal{P}, Y) \subseteq f \operatorname{Hom}(C, Y)$, then $Y$ is one of the submodules $Y^{\prime}$ with $u \operatorname{Hom}\left(C, \mathcal{P}, Y^{\prime}\right) \subseteq f \operatorname{Hom}(C, X)$ and therefore the image of $f$ contains $Y$, 
thus is equal to $Y$. This shows: If $f$ is right $C$-determined, then $f$ is surjective if and only if $\operatorname{Hom}(C, \mathcal{P}, Y) \subseteq f \operatorname{Hom}(C, X)$.

Corollary 8.4 Let $C, Y$ be modules.

(a) All maps in ${ }^{C}[\rightarrow Y\rangle$ are epimorphisms if and only of $\operatorname{Hom}(C, \mathcal{P}, Y)=0$.

(b) $\operatorname{Hom}(C, \mathcal{P}, Y)=\operatorname{Hom}(C, Y)$ if and only if the only element $[f\rangle$ in ${ }^{C}[\rightarrow Y\rangle$ with $f$ surjective is the right equivalence class of the identity map $Y \rightarrow Y$, if and only if any surjective map ending in $Y$ with kernel in add $\tau C$ splits.

\section{Kernels with injective dimension at most 1 .}

Proposition 8.5 Let $K$ be a module and $C=\tau^{-} K$. The following conditions are equivalent:

(i) The injective dimension of $K$ is at most 1.

(ii) If $Y$ is any module, then all maps in ${ }^{C}[\rightarrow Y\rangle$ are epimorphisms.

(iii) We have $\operatorname{Hom}(C, \mathcal{P}, Y)=0$ for all modules $Y$.

Proof Recall from [33], 2.4 that $K$ has injective dimension at most 1 if and only if $\operatorname{Hom}(C, \Lambda)=0$. Thus, if $K$ has injective dimension at most 1 and $Y$ is an arbitrary module, then $\operatorname{Hom}(C, \mathcal{P}, Y)=0$, this shows that (i) implies (iii). Conversely, assume the condition (iii), thus $\operatorname{Hom}(C, \mathcal{P}, Y)=0$ for all modules $Y$. If the injective dimension of $K$ would be at least 2 , then $\operatorname{Hom}(C, \Lambda) \neq 0$. But $\operatorname{Hom}(C, \mathcal{P}, \Lambda)=\operatorname{Hom}(C, \Lambda)$. This contradiction shows that (iii) implies (i). For the equivalence of (ii) and (iii) see Corollary 8.4(a).

Corollary 8.6 Let $\Lambda$ be hereditary and $C$ a module without any indecomposable projective direct summand. Then any right $C$-determined morphism $f: X \rightarrow Y$ is an epimorphism.

Proof Let $K=\tau C$. Since $C$ has no indecomposable projective direct summand, it follows that $C=\tau^{-} K$. Since $\Lambda$ is hereditary, the injective dimension of any module is at most 1 . Since the injective dimension of $K$ is at most 1 , it follows from the proposition that all right $C$-determined maps are epimorphisms.

Of course, we also can show directly that $\operatorname{Hom}(C, \mathcal{P}, Y)=0$. Namely, let $g: C \rightarrow$ $Y$ be in $\operatorname{Hom}(C, \mathcal{P}, Y)$. Then $g=g_{2} g_{1}$ with $g_{1}: C \rightarrow P$, where $P$ is a projective module. The image $P^{\prime}$ of $g_{1}$ is a submodule of $P$, thus, since $\Lambda$ is hereditary, the module $P^{\prime}$ is also projective. Thus, we have a surjective map $C \rightarrow P^{\prime}$ with $P^{\prime}$ projective. Such a map splits. This shows that $P^{\prime}$ is isomorphic to a direct summand of $C$. It follows that $P^{\prime}=0$ and therefore $g=0$.

Riedtmann-Zwara degenerations. Recall that $M^{\prime}$ is a Riedtmann-Zwara degeneration of $M$ if and only if there is an exact sequence of the form

$$
0 \rightarrow K \rightarrow K \oplus M \rightarrow M^{\prime} \rightarrow 0
$$

or, equivalently, if and only if there is an exact sequence of the form

$$
0 \rightarrow M^{\prime} \rightarrow M \oplus L \rightarrow L \rightarrow 0
$$


(in both sequences we can assume that the maps $K \rightarrow K$ and $L \rightarrow L$, respectively, are in the radical).

In terms of the Auslander bijection, we may deal with these data in several different ways: namely, we may look at the right equivalence classes of both $\left[M \rightarrow M^{\prime}\right\rangle$ and $\left[K \oplus M \rightarrow M^{\prime}\right\rangle$ in $\left[\rightarrow M^{\prime}\right\rangle$ as well as at the right equivalence class $\left[M^{\prime} \rightarrow M\right\rangle$ in $[\rightarrow M\rangle$. In case we deal with $\left[K \oplus M \rightarrow M^{\prime}\right\rangle$, one should be aware that this map $\left[K \oplus M \rightarrow M^{\prime}\right\rangle$ is the join of the two maps $\left[K \rightarrow M^{\prime}\right\rangle$ and $\left[M \rightarrow M^{\prime}\right\rangle$ in $\left[\rightarrow M^{\prime}\right\rangle$.

In addition, we also may concentrate on the possible maps $K \rightarrow K$ and $L \rightarrow L$ (sometimes called steering maps).

When dealing with epimorphisms in ${ }^{C}[\rightarrow Y\rangle$, Riedtmann-Zwara degenerations play a decisive role, as the following proposition shows:

Proposition 8.7 Let $f: X \rightarrow Y$ and $f^{\prime}: X^{\prime} \rightarrow Y$ be epimorphisms with isomorphic kernels. If $[f\rangle \leq\left[f^{\prime}\right\rangle$, then $X^{\prime}$ is a Riedtmann-Zwara degeneration of $X$.

Proof Let $h: X \rightarrow X^{\prime}$ with $f=f^{\prime} h$. Let $u: K \rightarrow X$ and $u^{\prime}: K \rightarrow X^{\prime}$ be the kernel maps. Since $f=f^{\prime} h$, there is $h^{\prime}: K \rightarrow K$ such that $u^{\prime} h^{\prime}=h u$, thus we deal with the following commutative diagram with exact sequences:

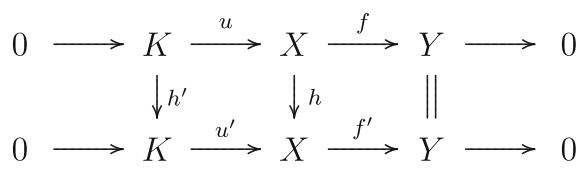

The diagram shows that the lower exact sequence is induced from the upper one by $h^{\prime}$. But this means that the following sequence is exact:

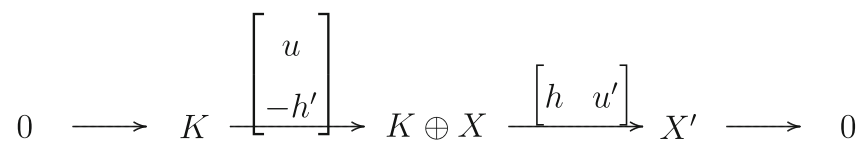

This is a Riedtmann-Zwara sequence, thus $X^{\prime}$ is a Riedtmann-Zwara degeneration of $X$.

Example 8 Let $\Lambda$ be given by the quiver with one loop $\alpha$ at the vertex $a$ and an arrow $b \rightarrow a$, with the relation $\alpha^{2}=0$.

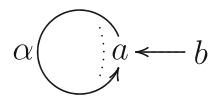

Let $K=P(a)$ and $Y=S(b)$, thus $\operatorname{dim} \operatorname{Ext}^{1}(Y, K)=2$. The module $C=\tau^{-} K$ is of length 4 with socle $a$ and top $b \oplus b$, thus $\operatorname{dim} \operatorname{Hom}(C, Y)=2$. Note that $\Gamma(C)=\operatorname{End}(C)^{\mathrm{op}}=k[t] / t^{2}$ and that $\operatorname{Hom}(C, Y)$ as a $\Gamma(C)$-module is cyclic.

The universal cover of the Auslander-Reiten quiver of $\Lambda$ looks as follows: 


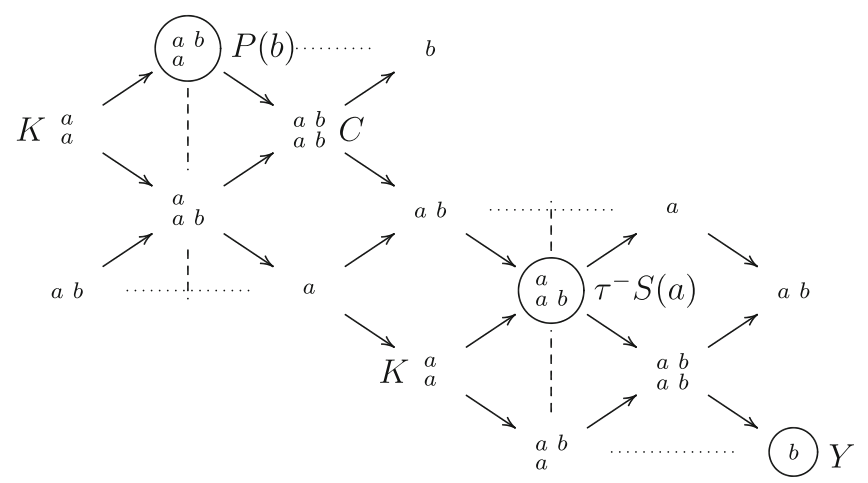

The region in-between the dashed lines is a fundamental domain of the AuslanderReiten quiver of $\Lambda$; the Auslander-Reiten quiver of $\Lambda$ is obtained by identifying these lines in order to form a Moebius strip.

The encircled vertices in the Auslander-Reiten quiver yield ${ }^{C}[\rightarrow Y\rangle$. Here is ${ }^{C}[\rightarrow Y\rangle$ as well as $\mathcal{S} \operatorname{Hom}(C, Y)$ :

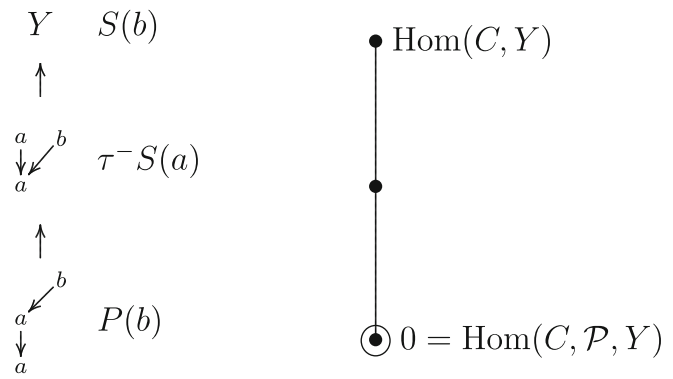

\section{Modules $K$ with semisimple endomorphism ring}

We start with a well-known characterization of such modules.

Lemma 9.1 Let $K$ be a module. The following conditions are equivalent:

(i) The endomorphism ring of $K$ is semisimple.

(ii) There are pairwise orthogonal bricks $K_{1}, \ldots, K_{n}$ such that add $K=$ add $\left\{K_{1}, \ldots, K_{n}\right\}$.

Proposition 9.2 Let $K$ be a module with semisimple endomorphism ring. Let $f=$ $f^{\prime} h$, where $f, f^{\prime}$ are right minimal epimorphisms with kernels in add $K$, starting at the same module $X$. Then $h$ is surjective and its kernel belongs to add $K$.

Proof By assumption, there is a commutative diagram with exact rows

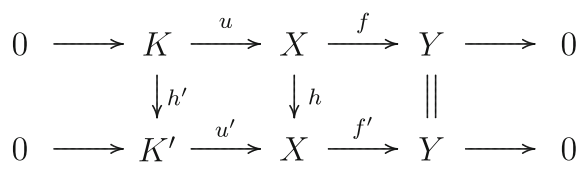


with inclusion maps $u, u^{\prime}$ such that both $K, K^{\prime}$ belong to add $K$. Since $K, K^{\prime}$ belong to add $K$ and the endomorphism ring of $K$ is semisimple, there is a submodule $K^{\prime \prime}$ of $K^{\prime}$ such that $K^{\prime}=\operatorname{Im}\left(h^{\prime}\right) \oplus K^{\prime \prime}$. Let us denote by $u^{\prime \prime}: K^{\prime \prime} \rightarrow K^{\prime}$ the inclusion map and by $q^{\prime}: K^{\prime} \rightarrow K^{\prime} / \operatorname{Im}\left(h^{\prime}\right)=K^{\prime \prime}$ the canonical projection, thus $q^{\prime} u^{\prime \prime}=1_{K^{\prime \prime}}$. Now $q^{\prime}: K^{\prime} \rightarrow K^{\prime \prime}$ is the cokernel of $h^{\prime}$, and we can identify $K^{\prime \prime}$ and we can complete the diagram above by inserting the cokernels of $h$ and $h^{\prime}$ as follows:

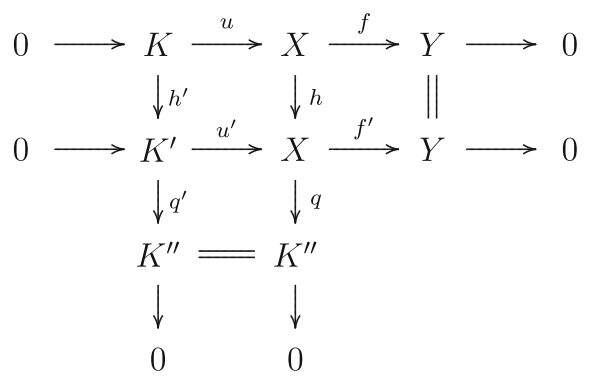

Since $q u^{\prime}=q^{\prime}$, we see that $q u^{\prime} u^{\prime \prime}=q^{\prime} u^{\prime \prime}=1_{K^{\prime \prime}}$, thus $K^{\prime \prime}$ is a direct summand of $X$ which lies inside the kernel of $f^{\prime}$. Since we assume that $f^{\prime}$ is right minimal, it follows that $K^{\prime \prime}=0$, thus $h^{\prime}$ is surjective. Therefore also $h$ is surjective.

On the other hand, the kernel of $h$ can be identified with the kernel of $h^{\prime}$, and again using that $K, K^{\prime}$ belong to add $K$ and that the endomorphism ring of $K$ is semisimple, we see that the kernel of $h$ belongs to add $K$.

Proposition 9.3 Let $K$ be a module and assume that the endomorphism ring of $K$ is semisimple. Let $C=\tau^{-} K$ and assume that $\operatorname{Hom}(C, \mathcal{P}, Y)=0$. If $f: X \rightarrow Y$ is right minimal and right $C$-determined, then $f$ is surjective and

$$
|f|_{C}=\mu(\operatorname{Ker}(f))
$$

where $\mu(M)$ is the Krull-Remak-Schmidt number (the number of direct summands when $M$ is written as a direct sum of indecomposable modules.

Also, $\eta_{C Y}^{-1}(0)$ is given by the universal extension from below

$$
0 \rightarrow K^{\prime} \rightarrow X \rightarrow Y \rightarrow 0
$$

with $K^{\prime} \in$ add $K$.

Proof Since we assume that $\operatorname{Hom}(C, \mathcal{P}, Y)=0$, all right minimal right $C$-determined maps $f: X \rightarrow Y$ are surjective, and the kernel of such a map is in add $K$. Let $|f|_{C}=n$ and consider a (maximal) chain

$$
X_{n} \stackrel{h_{n}}{\longrightarrow} X_{n-1} \stackrel{h_{n-1}}{\longrightarrow} \cdots \stackrel{h_{2}}{\longrightarrow} X_{1} \stackrel{h_{1}}{\longrightarrow} X_{0}=Y
$$

of non-invertible maps such that the compositions $f_{t}=h_{1} \cdots h_{t}$ for $1 \leq t \leq n$ are right minimal and right $C$-determined. Now all the maps $f_{t}$ are right minimal epimorphisms with kernels in add $K$, thus, according to proposition 7.2, also the maps $h_{t}$ are 
epimorphisms with kernels $K_{t}$ in add $K$. Since we assume that the endomorphism ring of $K$ is semisimple, we see that the kernel of $f_{t}$ is just $\bigoplus_{i=1}^{t} K_{t}$. Now let $K_{t}=K^{\prime} \oplus K^{\prime \prime}$ be a direct decomposition with $K^{\prime}$ indecomposable, and let $h: X_{t} \rightarrow X_{t} / K^{\prime}$ be the canonical projection. Since $K^{\prime}$ is contained in the kernel of $h_{t}$, we can factor $h_{t}$ through $h$ and obtain a map $h_{t}^{\prime}: X_{t} / K^{\prime} \rightarrow X_{t-1}$ with kernel $X^{\prime \prime}$ such that $h_{t}=h_{t}^{\prime} h$. Altogether we have obtained a refinement

$X_{n} \stackrel{h_{n}}{\longrightarrow} X_{n-1} \stackrel{h_{n-1}}{\longrightarrow} \cdots \longrightarrow X_{t} \stackrel{h}{\longrightarrow} X_{t} / K^{\prime} \stackrel{h_{t}^{\prime}}{\longrightarrow} X_{t-1} \longrightarrow \cdots \stackrel{h_{2}}{\longrightarrow} X_{1} \stackrel{h_{1}}{\longrightarrow} X_{0}=Y$

with $h_{t}=h_{t}^{\prime} h$. Let $f^{\prime}=f_{t-1} h_{t}^{\prime}=h_{1} \cdots h_{t-1} h_{t}^{\prime}$. We apply Proposition 9.1 to $f_{t}$ and $f^{\prime}$. Note that $f_{t}=f_{t-1} h_{t}=f_{t-1} h_{t}^{\prime} h=f^{\prime} h$ and the corresponding map from the kernel of $f_{t}$ to the kernel of $f^{\prime}$ is just the split epimorphism $K_{t} \rightarrow K_{t} / K^{\prime}$. Thus 9.1 asserts that $f^{\prime}$ is right minimal (and of course also right $C$-determined). The maximality of the chain $\left(h_{1}, \ldots, h_{t}\right)$ implies that $h_{t}^{\prime}$ has to be invertible, thus $K^{\prime \prime}=0$. This shows that $K_{t}$ is indecomposable. Altogether, we see that $\mu\left(\operatorname{Ker}\left(f_{t}\right)\right)=t$, thus $\mu(\operatorname{Ker}(f))=n=|f|_{C}$.

The last assertion is obvious: if

$$
0 \longrightarrow K^{\prime} \longrightarrow X \stackrel{f}{\longrightarrow} Y \longrightarrow 0
$$

is the universal extension from below with $K^{\prime} \in$ add $K$, then $[f\rangle$ belongs to ${ }^{C}[\rightarrow Y\rangle$ and any other extension of $Y$ from below with kernel in add $K$ is induced from it. Thus $[f\rangle$ has to be the zero element of the lattice ${ }^{C}[\rightarrow Y\rangle$.

Corollary 9.4 Let $K$ be a module with injective dimension at most 1 and assume that the endomorphism ring of $K$ is semisimple. Let $C=\tau^{-} K$. If $f: X \rightarrow Y$ is right minimal and right $C$-determined, then $f$ is surjective and $|f|_{C}=\mu(\operatorname{Ker}(f))$. Also, $\eta_{C Y}^{-1}(0)$ is given by the universal extension from below using modules in add $K$.

Proof Combine Proposition 8.5 and Proposition 9.3.

Example 9 Consider the 3-subspace quiver

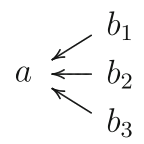

We consider the indecomposable modules which are neither projective, nor injective: these are the modules $N(i)=\tau Q\left(b_{i}\right)$, for $1 \leq i \leq 3$ and $M=\tau Q(a)$.

Let $Y=Q(a)$ and $C=N(1) \oplus N(2) \oplus N(3)$. Then ${ }^{C}[\rightarrow Q(a)\rangle$ looks as follows: 

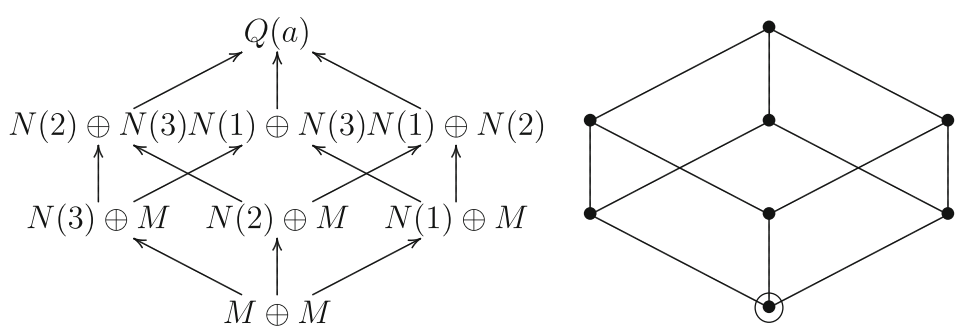

Note that here we have $K=\tau C=P\left(B_{1}\right) \oplus P\left(b_{2}\right) \oplus P\left(b_{3}\right)$.

\section{Comparison with Auslander-Reiten theory}

Let $C=Y$ be indecomposable and consider the Auslander bijection for $C=Y$ :

$$
{ }^{Y}[\rightarrow Y\rangle \longleftrightarrow \mathcal{S} \operatorname{Hom}(Y, Y)
$$

The subspace $\operatorname{rad}(Y, Y)$ of $\operatorname{Hom}(Y, Y)$ on the right corresponds to the right almost split map ending in $Y$. Two possible cases have to be distinguished:

If $Y=P$ is projective, then we get a morphism which is right determined by a projective module, thus we must get a monomorphism. Of course, what we obtain is just the embedding of the radical rad $P$ into $P$.

If $Y$ is not projective, then we get an epimorphism with kernel in add $\tau Y$. Actually, we get the epimorphism of the Auslander-Reiten sequence ending in $Y$, thus the kernel is precisely $\tau Y$.

What we see is that the minimal right almost split map ending in $Y$ is a waist in ${ }^{Y}[\rightarrow Y\rangle$ and it just corresponds to the waist $\operatorname{rad}(Y, Y) \subset \operatorname{End}(Y)$.

More generally, we have:

Proposition 10.1 Let $Y$ be indecomposable and $Y$ a direct summand of $C$, and consider

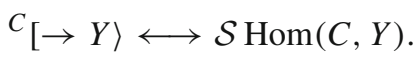

Then $\mathcal{S} \operatorname{Hom}(C, Y)$ is a local module (with maximal submodule $\operatorname{Im} \operatorname{Hom}(C, g)$, where $g$ is minimal right almost split ending in $Y$ ).

Proof Let $\tau Y \longrightarrow \mu Y \stackrel{g}{\longrightarrow} Y$ be the Auslander-Reiten sequence ending in $Y$. Then the right-equivalence class $[g\rangle$ of $g$ belongs to ${ }^{C}[\rightarrow Y\rangle$ and every map $X \rightarrow Y$ which is not a split epimorphism, factors through $g$. This means that every element of ${ }^{C}[\rightarrow Y\rangle$ different from the identity map $Y \rightarrow Y$ is less or equal to $[g\rangle$. This means that ${ }^{C}[\rightarrow Y\rangle$ has a unique maximal submodule, namely $\operatorname{Hom}(C, g)$.

The Auslander-Reiten formula $\operatorname{Ext}^{1}(Y, K) \simeq D \underline{\operatorname{Hom}}\left(\tau^{-} K, Y\right)$. The Auslander bijection provides a bijection between the submodules of $\underline{\operatorname{Hom}}\left(\tau^{-} K, Y\right)$ with the right equivalence classes of surjective maps $M \rightarrow Y$ with kernel in $\operatorname{add}(K)$ (namely, the submodules of $\underline{\operatorname{Hom}}\left(\tau^{-} K, Y\right)$ just correspond bijectively to the submodules of 
$\operatorname{Hom}\left(\tau^{-} K, Y\right)$ which contain $\left.\operatorname{Hom}\left(\tau^{-} K, \mathcal{P}, Y\right)\right)$. Consider the following triangle:

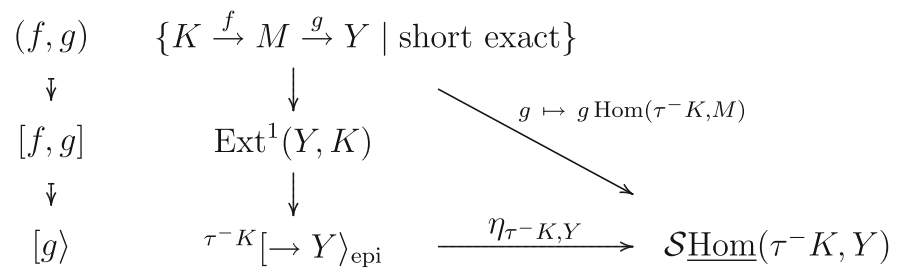

Here, the two left hand columns concern the canonical way of attaching to a short exact sequence $\epsilon=(f, g)$ the corresponding element $[\epsilon]=[f, g]$ in $\operatorname{Ext}^{1}$; if $(f, g)$ and $\left(f^{\prime}, g^{\prime}\right)$ are short exact sequences with $[f, g]=\left[f^{\prime}, g^{\prime}\right]$ in Ext ${ }^{1}$, then the maps $f, f^{\prime}$ are right equivalent, thus $[f, g] \mapsto[g\rangle$ is a well-defined map $\operatorname{Ext}^{1}(Y, K) \rightarrow{ }^{\tau^{-} K}[\rightarrow Y\rangle_{\text {epi }}$ and we obtain a commutative triangle as shown.

Having another look at the left columns of the triangle, the reader should obverse that the split short exact sequence which yields the zero element of $\operatorname{Ext}^{1}$ gives the unit element of the lattice $\tau^{-} K[\rightarrow Y\rangle_{\text {epi }}$, namely the identity map $1: Y \rightarrow Y$.

Proposition 10.2 Let $g: M \rightarrow Y$ be surjective with kernel $K$ and $C=\tau^{-} K$. Then an element $h \in \operatorname{Hom}(C, Y)$ belongs to the set $\eta_{C, Y}(g)$ if and only if the induced sequence $h_{*}([f, g])$ splits. Also, the set $\eta_{C, Y}(g)$ is a $\Gamma(C)$-submodule of $\operatorname{Hom}(C, Y)$, where $\Gamma(C)=\operatorname{End}(C)^{o p}$.

Proof Given an element $h \in \operatorname{Hom}(C, Y)$, the induced sequence $h_{*}([f, g])$ splits if and only if $h$ factors through $g$. But we have already noted that $\eta_{C, Y}(g)$ is the set of all elements $h \in \operatorname{Hom}(C, Y)$ which factor through $g$. And we know that $\eta_{C, Y}(g)$ is a $\Gamma(C)$-submodule of $\operatorname{Hom}(C, Y)$.

Now let us invoke the Auslander-Reiten formula: $\operatorname{Ext}^{1}(Y, K) \simeq D \underline{\operatorname{Hom}}\left(\tau^{-} K, Y\right)$. Here we use that we deal with an artin algebra $\Lambda$, thus the center $k$ of $\Lambda$ is a (commutative) artinian ring and $D$ is the duality functor $\bmod k \rightarrow \bmod k$ given by a minimal cogenerator of $\bmod k$.

There are the following two horizontal bijections as well as the vertical map on the left:

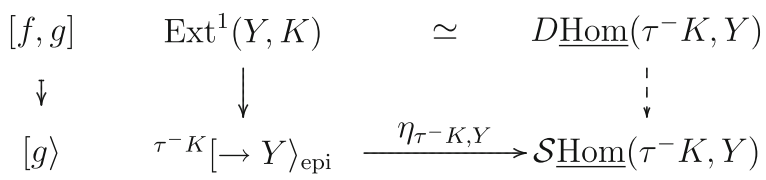

We have inserted a dashed arrow on the right which corresponds to the composition of the three given maps

$$
D \underline{\operatorname{Hom}}\left(\tau^{-} K, Y\right) \longrightarrow \operatorname{Ext}^{1}(Y, K) \longrightarrow \tau^{-} K[\rightarrow Y\rangle_{\text {epi }} \longrightarrow \mathcal{S H o m}\left(\tau^{-} K, Y\right)
$$

It seems to be of interest to describe in detail this composition! One may conjecture that here one attaches to a linear map $\alpha \in D \underline{\operatorname{Hom}}\left(\tau^{-} K, Y\right)$ the largest $\Gamma$-submodule of $\underline{\operatorname{Hom}}\left(\tau^{-} K, Y\right)$ lying in the kernel of $\alpha$. 
Let us now assume that $K$ is indecomposable so that add $K$ consists of direct sums of copies of $K$. Given a short exact sequence $K \stackrel{f}{\rightarrow} M \stackrel{g}{\rightarrow} Y$, the map $g$ is right minimal if and only if the sequence does not split. On the other hand, given $[g\rangle \in \epsilon^{\tau^{-} K}[\rightarrow Y\rangle_{\text {epi }}$ with $g$ right minimal, the kernel of $g$ is the direct sum of say $t$ copies of $K$ and $t \leq 1$ just means that $[g\rangle$ is obtained from a short exact sequence $K \stackrel{f}{\rightarrow} M \stackrel{g}{\rightarrow} Y$. It follows that under the assumption that $K$ is indecomposable, it is easy to identify the elements of $\mathcal{S} \operatorname{Hom}\left(\tau^{-} K, Y\right)$ which are images of the elements of $\operatorname{Ext}^{1}(Y, K)$ under $\eta_{\tau^{-} K, Y}$.

Comparison. In which way are the Auslander bijections better than the AuslanderReiten formula? What is the advantage of the Auslander bijection compared to the Auslander-Reiten formula?

1) We do not only deal with the set $\underline{\operatorname{Hom}}(C, Y)$ but with all of $\operatorname{Hom}(C, Y)$. To extend such a bijection as given by the Auslander-Reiten formula to a larger setting should always be of interest. But also note that the set $\operatorname{Hom}(C, Y)$ depends on the module category which we consider, not just on the modules themselves.

2) The duality is replaced by a covariant bijection.

3) The usual Auslander-Reiten picture concerns indecomposable modules, and almost split sequences, thus indecomposable modules and irreducible maps. In the language of the Auslander bijection, we only deal with $C=Y$ indecomposable and only with the submodule $\operatorname{rad}(C, C) \subset \operatorname{Hom}(C, C)$, whereas

- we should not restrict to indecomposable modules,

- and not to the condition $C=Y$,

- and we want to deal with all submodules of $\operatorname{Hom}(C, Y)$, not just the radical subspace.

Concerning the Auslander-Reiten-theory, there is an essential difference whether $C$ is projective or not. If $C$ is projective, we obtain an inclusion map, whereas if $C$ is not projective, then we obtain an extension. - This feature dominates also the Auslander bijections: First of all, there is the extreme case of $C$ being projective, then we consider submodules (and we consider arbitrarily ones, not just the radicals of the indecomposable projective modules). In general we deal with extensions ending in a submodule $Y^{\prime}$ of $Y$; if $C$ is a generator, then we deal with all possible extensions of submodules of $Y$ from below using modules in add $\tau C$.

4) The Auslander-Reiten theory only deals with the factor category of $\bmod \Lambda$ modulo the infinite radical. The Auslander bijection takes care of any morphism.

5) Families of modules do not play any role in the Auslander-Reiten theory. As we will see soon, families of modules are an essential features in the frame of the Auslander bijections.

\section{Families of modules}

\section{The modules present in ${ }^{C}[\rightarrow Y\rangle$ are of bounded length}

We say that a module $M$ is present in ${ }^{C}[\rightarrow Y\rangle$ provided there exists a right minimal map $f: M \rightarrow Y$ which is right $C$-determined; similarly, we say that $M$ is present 
in $^{C}[\rightarrow Y\rangle^{t}$ provided there exists a right minimal map $f: M \rightarrow Y$ which is right $C$-determined and $|f|_{C}=t$.

Proposition 11.1 There is a constant $\lambda=\lambda(\Lambda)$ such that for any pair of modules $C, Y$ with $C \neq 0$ and any right minimal right $C$-determined map $X \rightarrow Y$, we have

$$
|X| \leq \lambda|C|^{2}|Y|
$$

Proof Write $C=\bigoplus C_{i}$ with indecomposable direct summands $C_{i}$ and let $K_{i}=\tau C_{i}$. Note that $\left|K_{i}\right| \leq d^{2}\left|C_{i}\right|$, where $d=\left.\right|_{\Lambda} \Lambda \mid$ (see for example [30]). Of course, there is also the weaker bound $\left|K_{i}\right| \leq d^{2}|C|$.

Let $\Lambda$ be an artin $k$-algebra, where $k$ is a commutative artinian ring. Since there are only finitely many simple modules and since $\operatorname{Ext}^{1}(Y, X)$ is a $k$-module of finite length for all $\Lambda$-modules $X, Y$ of finite length, the length of the $k$-modules $\operatorname{Ext}^{1}\left(S, S^{\prime}\right)$, where $S, S^{\prime}$ are simple $\Lambda$-modules, is bounded, thus let $e$ be the maximum.

The long exact Hom-sequences imply that the length of the $k$-module $\operatorname{Ext}^{1}(Y, X)$ is bounded by $e|X||Y|$, for any $\Lambda$-modules $X, Y$ of finite length.

Now, let $f: X \rightarrow Y$ be right minimal and right $C$-determined. There is a short exact sequence $K^{\prime} \rightarrow X \rightarrow Y^{\prime}$ with $Y^{\prime}$ a submodule of $Y$, such that the module $K^{\prime}$ belongs to add $\tau C$ and such that $f$ is the composition of $X \rightarrow Y^{\prime}$ and the inclusion map $Y^{\prime} \rightarrow Y$. With $f$ also the map $X \rightarrow Y^{\prime}$ is right minimal.

Write $K^{\prime}=\bigoplus_{i=1}^{s} K_{i}^{t_{i}}$. The Ext-Lemma of [35] asserts that $t_{i}$ is bounded by the $k$-length of $\operatorname{Ext}^{1}\left(Y^{\prime}, K_{i}\right)$, since the map $X \rightarrow Y^{\prime}$ is right minimal. Thus

$$
t_{i} \leq e\left|K_{i}\right|\left|Y^{\prime}\right| \leq d^{2} e\left|C_{i}\right|\left|Y^{\prime}\right| \leq d^{2} e\left|C_{i}\right||Y|
$$

and therefore $\sum t_{i} \leq d^{2} e|C||Y|$. Thus

$$
\left|K^{\prime}\right|=\sum t_{i}\left|K_{i}\right| \leq \sum t_{i} d^{2}|C|=d^{2}|C| \sum t_{i} \leq d^{4} e|C|^{2}|Y|
$$

and therefore

$$
|X|=\left|K^{\prime}\right|+\left|Y^{\prime}\right| \leq d^{4} e|C|^{2}|Y|+|Y| \leq d^{4} e|C|^{2}|Y|+|C|^{2}|Y|=\left(d^{4} e+1\right)|C|^{2}|Y|
$$

(here we use that $C \neq 0$ ). Thus, let $\lambda=d^{4} e+1$.

There is the following converse:

Proposition 11.2 Let $Q=(D \Lambda)^{b}$. Then any module of length at most $b$ is present in $\Lambda_{[\rightarrow Q\rangle}$.

Proof Let $M$ be a module of length at most $b$, thus the socle soc $M$ of $M$ is a semisimple module of length at most $b$ and therefore a submodule of $Q=(D \Lambda)^{b}$. It follows that $M$ itself can be embedded into $Q$. Such an embedding $f: M \rightarrow Q$ is right minimal and right $\Lambda$-determined, thus $M$ is present in ${ }^{\Lambda}[\rightarrow Q\rangle$. 
Corollary 11.3 Let $\mathcal{M}$ be a set of modules. The modules in $\mathcal{M}$ are of bounded length if and only if there exist modules $C, Y$ such that any module $M$ in $\mathcal{M}$ is present in $C_{[\rightarrow Y\rangle \text {. }}$

Proof If all the modules in $\mathcal{M}$ are present in ${ }^{C}[\rightarrow Y\rangle$, then they have to be of length at most $\lambda(\Lambda)|C|^{2}|Y|$, thus of bounded length, see Proposition 11.1. Conversely, if the modules in $\mathcal{M}$ are of length at most $b$, then they are present in ${ }^{\Lambda}[\rightarrow Q\rangle$ where $Q=(D \Lambda)^{b}$, according to Proposition 11.2.

Remark Modules versus Morphisms. As we have seen, given any infinite set $\mathcal{M}$ of modules of bounded length, there are modules $C, Y$ such that all the modules in $\mathcal{M}$ are present in ${ }^{C}[\rightarrow Y\rangle$. On the other hand, given modules $X, Y$, one cannot expect that the right equivalence classes $[f\rangle$ of all (or at least infinitely many) non-zero morphisms $f: X \rightarrow Y$ belong to some ${ }^{C}[\rightarrow Y\rangle$, since the kernels of these maps $f$ may belong to infinitely many isomorphism classes.

\section{Minimal infinite families}

Recall that a Krull-Remak-Schmidt category $\mathcal{C}$ is said to be finite provided the number of isomorphism classes of indecomposable objects in $\mathcal{C}$ is finite, otherwise $\mathcal{C}$ is said to be infinite.

Let $\mathcal{M}$ be a family of modules. We say that $\mathcal{M}$ is minimal infinite provided add $\mathcal{M}$ is infinite whereas add $\mathcal{M}^{\prime}$ is finite, where $\mathcal{M}^{\prime}$ is the set of modules $M^{\prime}$ which are proper submodules or proper factor modules of modules in $\mathcal{M}$.

Lemma 12.1 If $\mathcal{M}$ is a minimal infinite family of modules (not necessarily of the same length), then there is an infinite subset $\mathcal{N} \subseteq \mathcal{M}$ which consists of pairwise non-isomorphic indecomposable modules of fixed length.

Of course, $\mathcal{N}$ is again minimal infinite.

Proof Since add $\mathcal{M}$ is infinite, there is a sequence of modules $M_{i} \in \mathcal{M}$ with $i \in \mathbb{N}$ such that $M_{i}$ does not belong to add $\left\{M_{1}, \ldots, M_{i-1}\right\}$ for all $i$. Write $M_{i}=N_{i} \oplus N_{i}^{\prime}$ such that $N_{i}$ is indecomposable and does not belong to add $\left\{M_{1}, \ldots, M_{i-1}\right\}$. It follows that the modules $N_{1}, N_{2}, \ldots$ are indecomposable and pairwise non-isomorphic. Let $I$ be the set of natural numbers such that $N_{i}^{\prime} \neq 0$. If $i \in I$, then $N_{i}$ is a proper submodule of $M_{i}$, thus belongs to add $\mathcal{M}^{\prime}$. Since add $\mathcal{M}^{\prime}$ is finite and the modules $N_{i}$ with $i \in I$ are indecomposable and pairwise non-isomorphic, it follows that $I$ is finite. Let $\mathcal{N}$ be the set of modules $N_{i}$ with $i \notin I$, then $\mathcal{N}$ is an infinite subset of $\mathcal{M}$ and consists of pairwise non-isomorphic indecomposable modules.

It is easy to see that the modules in a minimal infinite family of indecomposable modules are of bounded length. Namely, according to [36] (as well as [37]), any indecomposable module $M$ of length at least 2 has an indecomposable proper submodule of length at least $\frac{1}{p q}|M|$, where $p$ is the maximal length of an indecomposable projective module, $q$ the maximal length of an indecomposable injective module. Thus, if the modules in $\mathcal{N}$ are indecomposable, but not of bounded length, then we find indecomposable submodules of modules in $\mathcal{N}$ which are of arbitrarily large length. 
Thus, assume that the modules in $\mathcal{N}$ are of length at most $b$. Then there are infinitely many modules in $\mathcal{N}$ of fixed length $b^{\prime}$, for some $1 \leq b^{\prime} \leq b$.

Proposition 12.2 Let $\mathcal{M}$ be a minimal infinite family. Then there are indecomposable non-projective modules $C, Y$ and infinitely many indecomposable modules $M_{i} \in \mathcal{M}$ with short exact sequences

$$
0 \rightarrow \tau C \longrightarrow M_{i} \stackrel{f_{i}}{\longrightarrow} Y \rightarrow 0
$$

such that $f_{i}$ is a co-Gabriel-Roiter projection. All these equivalence classes $\left[f_{i}\right\rangle$ belong to ${ }^{C}[\rightarrow Y\rangle^{1}$.

Proof According to Lemma 12.1, we can assume that all the modules in $\mathcal{M}$ are indecomposable and of fixed length $b$. Let $\mathcal{M}^{\prime}$ be the set of modules $M^{\prime}$ which are proper submodules or proper factor modules of modules in $\mathcal{M}$. By assumption, there are only finitely many isomorphism classes of modules in $\mathcal{M}^{\prime}$. Since there are only finitely many simple modules, we must have $b \geq 2$, thus any $M \in \mathcal{M}$ has a co-GabrielRoiter factor-module $Q_{M}=M / K_{M}$; here, $K_{M}$ is a submodule of $M$. Note that $K_{M}$ is a proper non-zero submodule, that $Y_{M}$ is a proper factor module of $M$, and that both modules $K_{M}$ and $Y_{M}=M / K_{M}$ are indecomposable. Of course, both $K_{M}$ and $Y_{M}$ belong to $\mathcal{M}^{\prime}$. Since there are (up to isomorphism) only finitely many pairs $(K, Y)$ in $\left(\mathcal{M}^{\prime}\right)^{2}$, it follows that there is a pair $(K, M)$ such that there are infinitely many pairwise non-isomorphic indecomposable modules $M$ with $K=K_{M}$ and $Y=Y_{M}$. The exact sequences $0 \rightarrow K \rightarrow M \rightarrow Y \rightarrow 0$ with $M$ indecomposable show that $K$ cannot be injective, thus $C=\tau^{-} K$ is again indecomposable. This completes the proof of the proposition.

It remains to observe that $\left[f_{i}\right\rangle$ belongs to ${ }^{C}[\rightarrow Y\rangle^{1}$, but this has been shown in Corollary 7.6.

The lattices ${ }^{C}[\rightarrow Y\rangle$ which arise in this way can have arbitrarily large height, as the following examples show.

Example 10 Let $\Lambda$ be the $n$-Kronecker algebra, this is the path algebra of the quiver

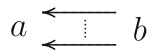

with $n$ arrows. Let $Y=S(b)$ and $C=\tau^{-} S(a)$. Then $C$ has dimension vector $\left(n^{2}-\right.$ $1, n)$, thus $\operatorname{dim} \operatorname{Hom}(C, Y)=n$. Since $\operatorname{End}(C)=k$, we see that $\mathcal{S} \operatorname{Hom}(C, Y)$ is of the form $\mathbb{G}(n)$ as exhibited in Sect. 19.7. The family of modules which we are interested in are the indecomposable modules $M$ of length 2. Given such a module $M$, there is an exact sequence

$$
0 \rightarrow S(a) \longrightarrow M \stackrel{f_{M}}{\longrightarrow} S(b) \rightarrow 0
$$

with $f_{M}$ a co-Gabriel-Roiter projection. As we will see in the next section, this family of maps $f_{M}$ is a cofork. 


\section{Forks and coforks}

We call a family of maps $\left(g_{i}: X \rightarrow M_{i}\right)_{i \in I}$ a fork provided for any finite subset $J \subseteq I$, the map $g_{J}=\left(g_{i}\right)_{i \in J}: X \rightarrow \bigoplus_{i \in J} M_{i}$ is left minimal. The dual notion will be that of a cofork, this is a set of maps $\left(f_{i}: M_{i} \rightarrow Y\right)_{i \in I}$ such that the direct sum map $f^{J}=\left(f_{i}\right)_{i \in J}: \bigoplus_{i \in J} M_{i} \rightarrow Y$ with $J$ any finite subset of $I$ is right minimal.

Lemma 13.1 Let $g_{i}: X \rightarrow M_{i}$ with $i \in I$ be a family of non-zero maps with indecomposable modules $M_{i}$. Then $\left(g_{i}: X \rightarrow M_{i}\right)_{i}$ is a fork if and only if $g_{i} \notin$ $\sum_{j \neq i} \operatorname{Hom}\left(M_{j}, M_{i}\right) g_{j}$ for all $i \in I$.

Proof First, assume that there is some $i$ with $g_{i} \in \sum_{j \neq i} \operatorname{Hom}\left(M_{j}, M_{i}\right) g_{j}$, say there is the subset $\{1,2, \ldots, t\} \subseteq I$ such that $g_{1} \in \sum_{j=2}^{t} \operatorname{Hom}\left(M_{j}, M_{1}\right) g_{j}$. Thus, for $2 \leq j \leq t$ there are maps $p_{j}: M_{j} \rightarrow M_{1}$ such that $g_{1}=\sum_{j=2}^{t} p_{j} g_{j}$. We want to show that the map $g_{J}: X \rightarrow M=\bigoplus_{i=1}^{t} M_{i}$ is not left minimal. Let $N$ be the kernel of the map

$$
\left(-1, p_{2}, \ldots, p_{t}\right): \bigoplus_{i=1}^{t} M_{i} \longrightarrow M_{1}
$$

It is easy to check that $M$ is the direct sum of $M_{1}$ and $N$. But the equality $-g_{1}+$ $\sum_{j=2}^{t} p_{j} g_{j}$ shows that the image of $g_{J}$ is contained in $N$. Since the image of $g_{J}$ is contained in a proper direct summand of $M$, we see that $g_{J}$ is not left minimal.

Conversely, assume that $g_{i} \notin \sum_{j \neq i} \operatorname{Hom}\left(M_{j}, M_{i}\right) g_{j}$ for all $i \in I$. We want to show that for any finite subset $J \subseteq I$, the map $g_{J}: X \rightarrow \bigoplus_{i \in J} M_{i}$ is left minimal. If $J$ is empty, then we deal with the zero map $X \rightarrow 0$ which of course is left minimal. If $J$ consists of the single element $i$, then we deal with $g_{i}: X \rightarrow M_{i}$. Since $g_{i} \neq 0$ and $M_{i}$ is indecomposable, the map $g_{i}$ is left minimal. Thus we can assume that $J$ contains $t \geq 2$ elements, say $J=\{1,2, \ldots, t\}$. Assume that the map $g_{J}: X \rightarrow M$ with $M=\bigoplus_{i=1}^{t} M_{i}$ is not left minimal. Then there is a proper direct decomposition $M=N \oplus N^{\prime}$ such that the image of $g_{J}$ is contained in $N$. We can assume that $N^{\prime}$ is indecomposable, thus isomorphic to some $M_{i}$, say to $M_{1}$. Let $p: M \rightarrow N^{\prime}=M_{1}$ be the projection with kernel $N$, write $p=\left(p_{i}\right)_{i}$ with $p_{i}: M_{i} \rightarrow M_{1}$. Note that $p_{1}$ has to be an isomorphism. Replacing any $p_{i}$ by $\left(p_{1}\right)^{-1} p_{i}$, we can assume that $p_{1}=1$. Since the image of $g_{J}$ is contained in the kernel $N$ of $p$, we have $\sum_{i=1}^{t} p_{i} g_{i}=0$, thus $g_{1}=-\sum_{i=2}^{t} p_{i} g_{i}$. This completes the proof.

We may use forks and coforks in order to construct inductively families of modules:

Proposition 13.2 Let $\left(f_{i}: M_{i} \rightarrow Y\right)_{i \in I}$ be an infinite cofork, with indecomposable modules $M_{i}$ which have the same Gabriel-Roiter measure $\gamma_{0}$. Let

$$
\mathcal{K}=\operatorname{add}\left\{\operatorname{Ker}\left(f^{J}\right)|J \subseteq I,| J \mid<\infty\right\}
$$

Then $\mathcal{K}$ is infinite, all indecomposable modules $K$ in $\mathcal{K}$ are cogenerated by $\left\{M_{i} \mid i \in I\right\}$ and have Gabriel-Roiter measure $\gamma(K)<\gamma_{0}$. 
Before we give the proof, let us note the following consequence: either $\mathcal{K}$ will contain indecomposable modules of arbitrarily large length, or else the indecomposable modules in $\mathcal{K}$ are of bounded length. In the latter case, there is an infinite subset of indecomposable modules $K_{i}$ in $\mathcal{K}$ all of which have the same Gabriel-Roiter measure $\gamma_{1}<\gamma_{0}$.

Proof First, assume that $\mathcal{K}=$ add $K$ for some module $K$, let $C=\tau^{-} K$. For any natural number $t$, we may choose a subset $J$ of $I$ of cardinality $t$. Then the module $M_{J}=\bigoplus_{i \in J} M_{i}$ is present in ${ }^{C}[\rightarrow Y\rangle$. But the modules which are present in ${ }^{C}[\rightarrow Y\rangle$ are of bounded length, whereas $\left|M_{J}\right| \geq t$. This contradiction shows that $\mathcal{K}$ is infinite.

Let $K$ be an indecomposable module in $\mathcal{K}$, say a direct summand of the kernel $K_{J}$ of $f^{J}: M_{J} \rightarrow Y$. Assume that $\gamma(K) \geq \gamma_{0}$. This implies that the inclusion map $K \subseteq K_{J} \subset M_{J}$ splits. But this contradicts the fact that the map $f^{J}: M_{J} \rightarrow Y$ is right minimal.

Let us present two different ways for obtaining forks:

Proposition 13.3 (Gabriel-Roiter forks) Let $M_{i}$ with $i \in I$ be pairwise nonisomorphic indecomposable modules of fixed length with isomorphic Gabriel-Roiter submodule $U$, say with embeddings $u_{i}: U \rightarrow M_{i}$. Then the family $\left(u_{i}: U \rightarrow M_{i}\right)_{i \in I}$ is a fork.

Proof The maps $u_{i}: U \rightarrow M_{i}$ are non-zero maps and the modules $M_{i}$ are indecomposable. Thus, if the family $\left(u_{i}: U \rightarrow M_{i}\right)_{i}$ is not a fork, then Lemma 13.1 asserts that there is some $i$ with $u_{i} \in \sum_{j \neq 1} \operatorname{Hom}\left(M_{j}, M_{1}\right) f_{j}$, thus we can assume that there is the subset $\{1,2, \ldots, t\} \subseteq I$ and maps $p_{j}: M_{j} \rightarrow M_{1}$ for $2 \leq j \leq t$ such that $u_{1}=\sum_{j=2}^{t} p_{j} u_{j}$.

Let $M^{\prime \prime}=\bigoplus_{j=2}^{t} M_{j}$. For $2 \leq j \leq t$, consider the submodule $p_{j} u_{j}(U)$ of $M_{1}$. It is a proper submodule of $M_{1}$, thus $\gamma\left(p_{j} u_{j}(U)\right) \leq \gamma(U)$, since $U$ is a Gabriel-Roiter submodule of $M_{1}$. On the other hand, the image of $\left(p_{j} u_{j}\right)_{j=2}^{t}: U \rightarrow M^{\prime \prime}$ is contained in $\bigoplus_{j=2}^{t} p_{j} u_{j}(U)$. Since $u_{1}=\sum_{j=2}^{t} p_{j} u_{j}$, this map $\left(p_{j} u_{j}\right)_{j=2}^{t}$ is injective, thus $U$ is a submodule of $\bigoplus_{j=2}^{t} p_{j} u_{j}(U)$. It follows that $\gamma(U) \leq \max \gamma\left(p_{j} u_{j}(U)\right)$. Thus there is some $s$ with $2 \leq s \leq t$ such that $\gamma(U)=\gamma\left(p_{s} u_{s}(U)\right)$. Since $U$ is indecomposable, $U$ has to be a direct summand of $p_{s} u_{s}(U)$. But $p_{s} u_{s}(U)$ is a factor module of $U$, thus $p_{s} u_{s}: U \rightarrow p_{s} u_{s}(U)$ is an isomorphism. As a consequence, $u_{s}$ is a split monomorphism. But this is impossible, since $u_{s}$ is the inclusion of a Gabriel-Roiter submodule.

Let us add the dual assertion.

Proposition 13.4 (co-Gabriel-Roiter coforks) Let $M_{i}$ with $i \in I$ be pairwise nonisomorphic indecomposable modules of fixed length with isomorphic co-GabrielRoiter factor modules $Y$, say with projections $v_{i}: M_{i} \rightarrow Y$. Then the family $\left(v_{i}: M_{i} \rightarrow Y\right)_{i \in I}$ is a cofork.

Remark Let us stress the following: Let $M_{i}$ with $i \in I$ be pairwise non-isomorphic indecomposable modules with Gabriel-Roiter submodules $U_{i} \subset M_{i}$ and assume that 
the modules $M_{i} / U_{i}$ are isomorphic, say to $Y$, with projection maps $f_{i}: M_{i} \rightarrow Y$. Then the family $\left(f_{i}: M_{i} \rightarrow Y\right)_{i \in I}$ is not necessarily a cofork. Namely, consider again example 8, and look at the projections $f: P(b) \rightarrow S(b)$ and $f^{\prime}: \tau^{-} S(a) \rightarrow S(b)$. As we mentioned already, the kernels of both maps are Gabriel-Roiter submodules. Since there is a factorization $f=f^{\prime} h$, the map $\left[f, f^{\prime}\right]: P(b) \oplus \tau^{-} S(a) \rightarrow S(b)$ is not right minimal.

Here is a consequence of Proposition 13.4 and Corollary 7.6.

Proposition 13.5 Let $M_{i}$ with $i \in I$ be pairwise non-isomorphic indecomposable modules with isomorphic co-Gabriel-Roiter factor modules $Y$, say with projections $v_{i}: M_{i} \rightarrow Y$ and assume that also the kernels are isomorphic, say isomorphic to $K$. Let $C=\tau^{-} K$. Then $\left(v_{i}: M_{i} \rightarrow Y\right)_{i \in I}$ is a cofork which belongs to ${ }^{C}[\rightarrow Y\rangle^{1}$.

A proof similar to Proposition 13.3 shows that starting with any infinite family $\mathcal{M}$ of indecomposable modules with fixed length, there are infinite forks consisting of maps $S \rightarrow M$, where $S$ is a simple module and $M \in \mathcal{M}$. A fork $\left(g_{i}: S \rightarrow M_{i}\right)_{i}$ with $S$ simple will be called a simple fork. Similarly, a cofork $\left(f_{i}: M_{i} \rightarrow S\right)_{i}$ with $S$ simple is called a simple cofork.

Proposition 13.6 Let $M_{i}$ with $i \in I$ be pairwise non-isomorphic indecomposable modules with fixed Gabriel-Roiter measure. Then there exists for every index $i \in I$ a simple submodule $S_{i}$ of $M_{i}$, say with inclusion map $u_{i}: S_{i} \rightarrow M_{i}$, such that for every simple module $S$ and $I(S)=\left\{i \in I \mid S_{i}=S\right\}$ the family $\left(u_{i}: S \rightarrow M_{i}\right)_{i \in I(S)}$ is a fork.

Proof Let us assume that the modules in $\mathcal{M}$ have length $b$. Of course, $b>1$. Consider a module $M_{i}$ with $i \in I$. It is not cogenerated by the remaining modules, thus the intersection of the kernels of all maps $\phi: M_{i} \rightarrow M_{j}$ with $j \neq i$ is non-zero. Let $S_{i}$ be a simple submodule of $M_{i}$ which is contained in this intersection (thus $\phi\left(S_{i}\right)=0$ for all maps $\phi: M_{i} \rightarrow M_{j}$ with $j \neq i$. Denote by $u_{i}: S_{i} \rightarrow M$ the inclusion map, thus $\left.\operatorname{Hom}\left(M_{i}, M_{j}\right) u_{i}\right)=0$ for all $j \neq i$. For any simple module $S$, let $I(S)=\left\{i \in I \mid S_{i}=S\right\}$.

In order to see that $\left(u_{i}: S \rightarrow M_{i}\right)_{i \in I(S)}$ is a fork, we have to show the following: For any finite subset $J$ of $I$, say $J=\{1,2, \ldots, t\}$, the map $u^{J}:\left(u_{i}\right)_{i}: S \rightarrow \bigoplus_{i=1}^{t} M_{i}$ is left minimal. Again, this is clear for $t=1$, since the maps $u_{i}$ are non-zero and the modules $M_{i}$ are indecomposable. Thus we can assume that $t \geq 2$. If the map $u^{J}$ is not left minimal, then, up to permutation of the indices, there are maps $p_{i}: M_{i} \rightarrow M_{1}$ with $2 \leq i \leq t$ such that $u_{1}=\sum_{i=2}^{t} p_{i} u_{i}$. However, by construction, $\operatorname{Hom}\left(M_{i}, M_{1}\right) u_{i}=0$ for $i \neq 1$, thus all the summands $p_{i} u_{i}$ with $2 \leq i \leq t$ are zero. Since $u_{1} \neq 0$, we obtain a contradiction.

Corollary 13.7 (Simple forks) Let $\mathcal{M}$ be an infinite set of pairwise non-isomorphic indecomposable modules of fixed length. Then there exists an infinite simple fork $\left(u_{i}: S \rightarrow M_{i}\right)_{i}$ such that all $M_{i}$ belong to $\mathcal{M}$.

Proof Since the modules in $\mathcal{M}$ are of bounded length, only finitely many GabrielRoiter measures occur, thus there is an infinite subset $\mathcal{M}^{\prime} \subseteq \mathcal{M}$ which consists of 
indecomposable modules with fixed Gabriel-Roiter measure. Now we apply Proposition 13.6. Since there are only finitely many simple modules, one of the forks $\left(u_{i}: S \rightarrow M_{i}\right)_{i \in I(S)}$ has to be infinite.

Again we add the dual assertion.

Corollary 13.8 (Simple coforks) Let $\mathcal{M}$ be an infinite set of pairwise non-isomorphic indecomposable modules of fixed length. Then there exists an infinite simple cofork $\left(v_{i}: M_{i} \rightarrow S\right)_{i}$ such that all $M_{i}$ belong to $\mathcal{M}$.

The setting developed here allows to provide a proof of the following result which first was established in [35]. Note that this result strengthens the assertion of the first Brauer-Thrall conjecture [41].

Corollary 13.9 (First Brauer-Thrall conjecture) Let $\mathcal{M}$ be an infinite set of indecomposable modules of a fixed length. Then there are indecomposable modules of arbitrarily large length which are cogenerated by modules in $\mathcal{M}$.

Proof Let $\mathcal{M}_{0}=\mathcal{M}$ and apply Corollary 13.8. Thus, there is a simple cofork $\left(v_{i}: M_{i} \rightarrow S\right)_{i}$ such that all $M_{i}$ belong to $\mathcal{M}_{0}$. Let $\mathcal{K}=\operatorname{add}\left\{\operatorname{Ker}\left(v^{J}\right)|J \subseteq I| J \mid,<\right.$ $\infty\}$. According to Proposition 13.2 we know that $\mathcal{K}$ is infinite, that all indecomposable modules $K$ in $\mathcal{K}$ are cogenerated by $\left\{M_{i} \mid i \in I\right\}$ and that they have Gabriel-Roiter measure $\gamma(K)<\gamma_{0}$. Now either the indecomposable modules in $\mathcal{K}$ are of unbounded length, then we are done. Or else they are of bounded length: then we find in $\mathcal{K}$ an infinite set $\mathcal{M}_{1}$ of indecomposable modules having the same Gabriel-Roiter measure, say $\gamma_{1}$ and $\gamma_{1}<\gamma_{0}$. Inductively, we construct a sequence of sets of indecomposable modules

$$
\mathcal{M}_{0}, \mathcal{M}_{1}, \ldots, \mathcal{M}_{i}
$$

such that the modules in $\mathcal{M}_{i}$ are cogenerated by $\mathcal{M}_{i-1}$ and have fixed Gabriel-Roiter measure $\gamma_{i}<\gamma_{i-1}$, for $i \geq 1$. The procedure stops in case there are indecomposable modules of unbounded length which are cogenerated by $\mathcal{M}_{i}$, and then these modules are cogenerated by $\mathcal{M}$. Otherwise the procedure can be continued indefinitely. But then we have constructed infinitely many sets $\mathcal{M}_{i}$ of indecomposable modules. Since the modules in $\mathcal{M}_{i}$ have Gabriel-Roiter measure $\gamma_{i}$ and the measures $\gamma_{i}$ are pairwise different, the modules in $\bigcup_{i} \mathcal{M}_{i}$ cannot be of bounded length. Also, the modules in any $\mathcal{M}_{i}$ are cogenerated by $\mathcal{M}$. This completes the proof.

\section{The Kronecker algebra}

Throughout this section, $\Lambda$ will be the Kronecker algebra as introduced already in Example 2. It is a very important artin algebra and a clear understanding of its module category $\bmod \Lambda$ seems to be of interest.

For all pairs $C, Y$ of indecomposable $\Lambda$-modules, we are going to describe the lattice ${ }^{C}[\rightarrow Y\rangle$ as well as all the modules present in ${ }^{C}[\rightarrow Y\rangle^{1}$, this is the subset of ${ }^{C}[\rightarrow Y\rangle$ of elements of right $C$-length 1 . 
Let us recall the structure of the category mod $\Lambda$ (see for example [33], or [5], section VIII.7). There are the preprojective and the preinjective modules, modules without an indecomposable direct summand which is preprojective or preinjective are said to be regular. For any $\Lambda$-module $M$, its defect is defined by $\delta(M)=\operatorname{dim} \operatorname{Hom}\left(M, Q_{0}\right)-$ $\operatorname{dim} \operatorname{Hom}\left(P_{0}, M\right)$. Any indecomposable preprojective $\Lambda$-module has defect -1 , the indecomposable preinjective modules have defect 1 , all the regular modules have defect 0 . There are countably many indecomposable preprojective modules, they are labeled $P_{i}$, and also countably many indecomposable preinjective modules, they are labeled $Q_{i}$; both $P_{i}$ and $Q_{i}$ have length $2 i+1$. The indecomposable regular modules are those modules which belong to stable Auslander-Reiten components, and all these components are stable tubes of rank 1 . The full subcategory $\mathcal{R}$ of all regular modules is abelian. By definition, the simple regular modules are the regular modules which are simple objects in this subcategory. Given any indecomposable regular module $R$, its endomorphism ring $\operatorname{End}(R)$ is a commutative ring (namely a ring of the form $k[T] /\langle f\rangle$, where $k[T]$ is the polynomial ring in one variable $T$ with coefficients in $k$ and $f$ is a power of an irreducible polynomial) and $\operatorname{dim} R=2 \operatorname{dim} \operatorname{End}(R)$.

As we have mentioned, we are interested in pairs $C, Y$ of indecomposable $\Lambda$ modules such that a family of modules is present in ${ }^{C}[\rightarrow Y\rangle$. It turns out that only the case of $C$ being preprojective, $Y$ being preinjective is relevant, as the following proposition shows.

Proposition 14.1 Let $\Lambda$ be the Kronecker algebra and $C, Y$ indecomposable $\Lambda$ modules. If $C$ is preprojective or preinjective, then ${ }^{C}[\rightarrow Y\rangle$ is a projective geometry. If $C$ is regular, then ${ }^{C}[\rightarrow Y\rangle$ is a chain.

By definition, a projective geometry $\mathbb{G}(d)$ over the field $k$ is the lattice of subspaces of the $k$-space of dimension $d$. The chain $\mathbb{I}(d)$ is the set of integers $i$ with $0 \leq i \leq d$ with the usual ordering. The labels have been chosen in such a way that the height of $\mathbb{G}(d)$ as well as of $\mathbb{I}(d)$ is just $d$.

The following table provides the precise data: Here, an indecomposable regular module of regular length $t$ and with regular socle $R$ is denoted by $R[t]$.

\begin{tabular}{|c|c|c|c|c|}
\hline row & $C$ & $Y$ & & ${ }^{C}[\rightarrow Y\rangle$ \\
\hline 1) & $P_{i}$ & $P_{j}$ & $(i \leq j)$ & $\mathbb{G}(j-i+1)$ \\
\hline 2) & $P_{i}$ & $R[t]$ & & $\mathbb{G}\left(\frac{1}{2} \operatorname{dim} R[t]\right)$ \\
\hline 3) & $P_{i}$ & $Q_{j}$ & & $\mathbb{G}(i+j)$ \\
\hline 4) & $R[s]$ & $R[t]$ & & $\mathbb{I}(\min (s, t))$ \\
\hline 5) & $R[s]$ & $Q_{j}$ & & $\mathbb{I}\left(\frac{1}{2} \operatorname{dim} R[s]\right)$ \\
\hline 6) & $Q_{i}$ & $Q_{j}$ & $(i \geq j)$ & $\mathbb{G}(i-j+1)$ \\
\hline
\end{tabular}

Let us stress that in row 4), the regular modules $C, Y$ are supposed to belong to the same tube, namely to the tube containing a fixed simple regular module $R$. For all pairs $C, Y$ of indecomposable Kronecker modules which are not contained in the table, one has $\operatorname{Hom}(C, Y)=0$, thus ${ }^{C}[\rightarrow Y\rangle$ consists of a single element.

Proof of proposition 14.1 First, let us calculate $\operatorname{dim} \operatorname{Hom}(C, Y)$. The reflection functors of [7] yield 


$$
\begin{aligned}
& \operatorname{Hom}\left(P_{i}, P_{j}\right)=\operatorname{dim} \operatorname{Hom}\left(P_{0}, P_{j-i}\right)=j-i+1 \\
& \operatorname{Hom}\left(P_{i}, R[t]\right)=\operatorname{dim} \operatorname{Hom}\left(P_{0}, R[t]\right)=\frac{1}{2} \operatorname{dim} R[t] \\
& \operatorname{Hom}\left(P_{i}, Q_{j}\right)=\operatorname{dim} \operatorname{Hom}\left(P_{0}, Q_{j+i}\right)=i+j \\
& \operatorname{Hom}\left(R[s], Q_{j}\right)=\operatorname{dim} \operatorname{Hom}\left(R[s], Q_{0}\right)=\frac{1}{2} \operatorname{dim} R[s] \\
& \operatorname{Hom}\left(Q_{i}, Q_{j}\right)=\operatorname{dim} \operatorname{Hom}\left(Q_{i-j}, Q_{0}\right)=i-j+1
\end{aligned}
$$

As we have mentioned, we have $\operatorname{dim} R=2 \operatorname{dim} \operatorname{End}(R)$. It follows that

$$
\operatorname{dim} \operatorname{Hom}\left(R[s], Q_{j}\right)=\frac{1}{2} \operatorname{dim} R[s]=s \operatorname{dim} \operatorname{End}(R) .
$$

Finally,

$$
\operatorname{dim} \operatorname{Hom}(R[s], R[t])=\min (s, t) \operatorname{dim} \operatorname{End}(R) .
$$

In case $C=P_{i}$ or $Q_{j}$ one has $\operatorname{End}(C)=k$, thus Auslander's Second Theorem asserts that ${ }^{C}[\rightarrow Y\rangle$ is of the form $\mathbb{G}(\operatorname{dim} \operatorname{Hom}(C, Y))$. This yields the rows 1), 2), 3) and 6) of the table.

It remains to look at the rows 4) and 5), thus we assume now that $C=R[s]$ and $Y=R[t]$ or $Y=Q_{j}$. We show that $\operatorname{Hom}(C, Y)$ is a cyclic $\Gamma(C)$-module, thus we have to find an element $g \in \operatorname{Hom}(C, Y)$ such that $g \operatorname{End}(C)=\operatorname{Hom}(C, Y)$, or, equivalently, such that $g(\operatorname{rad} \operatorname{End}(C))^{r-1} \neq 0$, where $r$ is the length of $\operatorname{Hom}(C, Y)$ (as a $\Gamma(C)$-module). Note that $\Gamma(C)$ is a local ring, thus there is a unique simple $\Gamma(C)$-module $S$. Since $S$ has $k$-dimension $\operatorname{dim} \operatorname{End}(R)$, the calculations above show that $r=s$ in case $Y=Q_{j}$ and $r=\min (s, t)$ in case $Y=R[t]$. On the other hand, $(\operatorname{rad} \operatorname{End}(C))^{r-1}$ is generated by any endomorphism of $R[s]$ with image $R[s-r+1]$. Thus let $p: R[s] \rightarrow R[s-r+1]$ be the canonical projection, $u: R[s-r+1] \rightarrow R[s]$ the canonical inclusion, then up generates $(\operatorname{rad} \operatorname{End}(C))^{r-1}$. Our aim is to exhibit $g: R[s] \rightarrow Y$ such that $g u p \neq 0$.

First, let $Y=R[t]$ and $s \leq t$. Then $r=\min (s, t)=s$ and $R[s-r+1]=R[1]=R$. Let $g: R[s] \rightarrow R[t]$ be the canonical inclusion, thus $g u: R \rightarrow R[t]$ is an inclusion, in particular non-zero, and therefore also gup $\neq 0$.

Second, let $Y=R[t]$ and $s>t$. Then $r=\min (s, t)=t$ and $R[s-r+1]=R[s-$ $t+1]$. Let $g: R[s] \rightarrow R[t]$ be the canonical projection. Then $g u: R[s-t+1] \rightarrow R[t]$ has image $R$ (since the kernel of $g$ is $R[s-t]$ ). This shows that $g u$ is non-zero, and therefore also gup $\neq 0$.

Finally, we have to deal with the case $Y=Q_{j}$. Take a non-zero map $g^{\prime}: R \rightarrow Q_{j}$. Since $\operatorname{Ext}^{1}\left(R[s] / R, Q_{j}\right)=0$, there exists $g: R[s] \rightarrow Q_{j}$ such that $g u=g^{\prime}$. Since $g u \neq 0$, it follows that also $g u p \neq 0$.

Thus, always we have found $g: R[s] \rightarrow Y$ such that $g u p \neq 0$. As a consequence, $\operatorname{Hom}(C, Y)$ is a cyclic $\Gamma(C)$-module. Since $\Gamma(C)$ is a local uniserial ring, it follows that $\mathcal{S} \operatorname{Hom}(C, Y)$ is of the form $\mathbb{I}(r)$, where $r$ is the length of $\operatorname{Hom}(C, Y)$. According to our calculations, $r=\min (s, t)$ in case $Y=R[t]$ and $r=\frac{1}{2} \operatorname{dim} R[s]$ in case $Y=Q_{j}$.

Thus we have verified the assertions presented in the table. If the pair $C, Y$ does not occur in the table, then it is well-known that $\operatorname{Hom}(C, Y)=0$, thus $\mathcal{S} \operatorname{Hom}(C, Y)$ 
consists of a single element and therefore is of the form $\mathbb{I}(0)=\mathbb{G}(0)$. This completes the proof.

The following assertion which has been shown in the proof will be of further interest:

Lemma 14.2 Any non-zero map $g^{\prime}: R \rightarrow Q_{j}$ can be extended to a map $g: R[t] \rightarrow$ $Q_{j}$, and any such $g: R[t] \rightarrow Q_{j}$ generates the $\Gamma(C)$-module $\operatorname{Hom}\left(R[t], Q_{j}\right)$.

Remark We have mentioned in Sect. 4 that both sides of the Auslander bijection concern maps with target $Y$, but that they invoke these maps in quite different ways. A nice illustration seems to be Proposition 14.1. The map $g: R[t] \rightarrow Q_{j}$ constructed there is a generator of the maximal submodule of $\operatorname{Hom}\left(R[t], Q_{j}\right)$ and is used in the proof of Lemma 14.2 in order to show that $\mathcal{S} \operatorname{Hom}\left(R[t], Q_{j}\right)$ is of the form $\mathbb{I}(d)$ for some $d$. On the other hand, in Proposition 14.9, we will consider the right equivalence class $[g\rangle$ as an element of $\left[\rightarrow Q_{j}\right\rangle$.

Proposition 14.3 Let $\Lambda$ be the Kronecker algebra, let $C, Y$ be indecomposable $\Lambda$ modules and $M$ an indecomposable direct summand of a module present in ${ }^{C}[\rightarrow Y\rangle$.

If $C, Y$ both are preprojective, also $M$ is preprojective. If $C, Y$ both are preinjective, also $M$ is preinjective. If both $C, Y$ belong to the tube $\mathcal{T}$, also $M$ belongs to $\mathcal{T}$.

If $C$ is preprojective and $Y$ belongs to the tube $\mathcal{T}$, then $M$ is preprojective or belongs to $\mathcal{T}$. If $C$ belongs to the tube $\mathcal{T}$ and $Y$ is preinjective, then $M$ is preinjective or belongs to the tube $\mathcal{T}$.

Proof First, assume that $Y$ is preprojective. If $f: X \rightarrow Y$ is right minimal, then $X$ has to be preprojecive. Thus, for any module $C$, all the modules present in ${ }^{C}[\rightarrow Y\rangle$ are preprojective.

Second, assume that $Y$ is regular, say belonging to the tube $\mathcal{T}$. Again, assume that $f: X \rightarrow Y$ is right minimal. Then $X$ is the direct sum of a preprojective module and a module in $\mathcal{T}$. Thus, for any module $C$, all the modules present in ${ }^{C}[\rightarrow Y\rangle$ are direct sums of preprojective modules and modules in $\mathcal{T}$.

Finally, assume that $Y$ is preinjective and $C$ is regular or preinjective. Let $f: X \rightarrow$ $Y$ be right minimal and right $C$-determined. Since $C$ has no indecomposable projective direct summand and $\Lambda$ is hereditary, we see that $f$ is surjective and its kernel is in add $K$ with $K=\tau C$. Now, if $C$ is preinjective, then also $K$ is preinjective and $X$ as an extension of a preinjective module by a preinjective module is preinjective again. On the other hand, if $C$ is regular, say belonging to the tube $\mathcal{T}$, then also $K$ belongs to $\mathcal{T}$. Since $X$ is an extension of a module in $\mathcal{T}$ by a preinjective module, it is the direct sum of a module in $\mathcal{T}$ and a preinjective module. This completes the proof.

Remark Let us stress that the cases $C=P_{i}, Y=R[t]$ and $C=R[s], Y=Q_{j}$ are not at all dual (as the consideration of $\operatorname{Hom}\left(P_{i}, R[t]\right)$ and $\operatorname{Hom}\left(R[t], Q_{j}\right)$ could suggest), but are of completely different nature. The reason is that we always consider $\operatorname{Hom}(C, Y)$ as a $\Gamma(C)$-module!

Of course, we have already seen that ${ }^{C}[\rightarrow Y\rangle$ is in the first case a projective geometry, in the second case a chain. But also if we look at the different layers, we encounter clear differences. In the chain case, all the elements of ${ }^{C}[\rightarrow Y\rangle$ are given by short exact sequences of the form $\left(R[s] \rightarrow M \rightarrow Q_{j}\right)$, thus by elements of 
$\operatorname{Ext}^{1}\left(Q_{j}, R[s]\right)$. In this case, we may interpret ${ }^{C}[\rightarrow Y\rangle$ as a display of the various orbits in $\operatorname{Ext}^{1}\left(Q_{j}, R[s]\right)$ with respect to the action of the automorphism group of $R[s]$. In contrast, in the projective geometry case, only the elements of ${ }^{C}[\rightarrow Y\rangle$ of right $C$-length at most 1 are given by short exact sequences of the form $\left(P_{i} \rightarrow M \rightarrow R[t]\right)$, whereas for the elements of right $C$-length at least 2, we need short exact sequences of the form $\left(P_{i}{ }^{a} \rightarrow M \rightarrow R[t]\right)$ with $a \geq 2$.

In the proof of Proposition 14.3, we have seen that for $C=P_{i}, Y=R[t]$, the modules $M$ present in ${ }^{C}[\rightarrow Y\rangle$ are direct sums of preprojective modules and modules in the tube which contains $\mathcal{T}$, and that for $C=R[s], Y=Q_{j}$ the modules present in ${ }^{C}[\rightarrow Y\rangle$ are direct sums of preinjective modules and modules in $\mathcal{T}$. However, in the first case the number of indecomposable preprojective direct summands of $M$ may be large, whereas in the second case there is just one direct summand of $M$ which is indecomposable preinjective.

Preprojective $C$, preinjective $Y$. Let us focus now the attention on ${ }^{C}[\rightarrow Y\rangle$, where $C$ is indecomposable preprojective and $Y$ is indecomposable preinjective. Here is the general behavior as seen in Proposition 14.1:

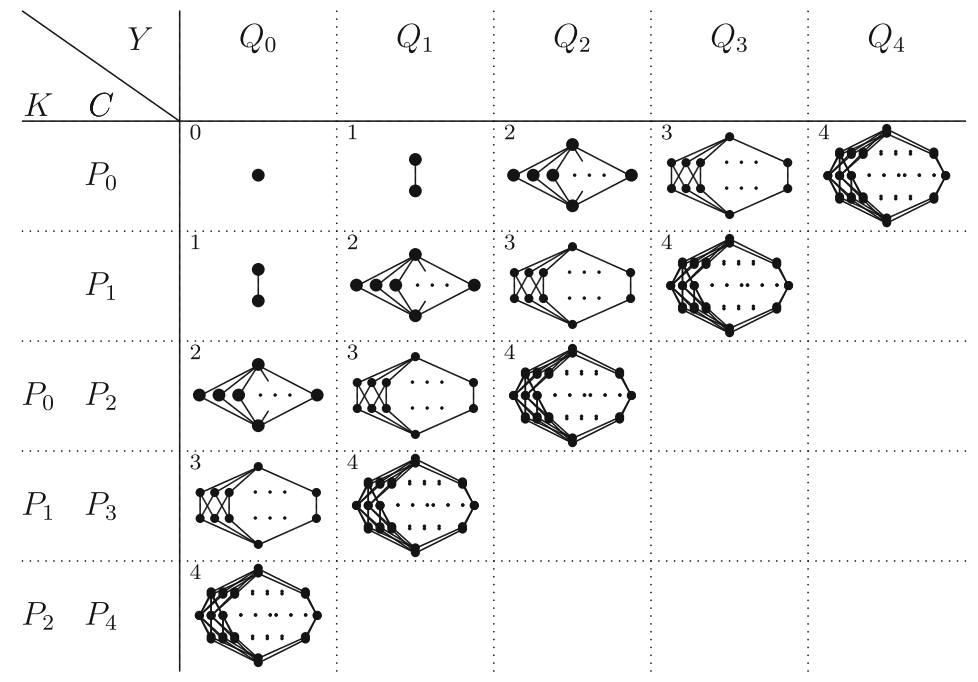

We want to know which modules are present in ${ }^{C}[\rightarrow Y\rangle^{1}$. As we will show, these are certain regular modules.

Lemma 14.4 Let $\Lambda$ be the Kronecker algebra and $M$ a regular module. The following conditions are equivalent.

(i) If $M=M^{\prime} \oplus M^{\prime \prime}$, then $\operatorname{Ext}^{1}\left(M^{\prime}, M^{\prime \prime}\right)=0$.

(ii) The regular socle of $M$ is multiplicity-free.

(ii*) The regular top of $M$ is multiplicity-free.

(iii) $M=\bigoplus_{i=1}^{n} M_{i}$, with indecomposable modules $M_{i}$ belonging to pairwise different tubes.

(iv) $\operatorname{End}(M)$ is commutative. 
Proof The equivalence of (ii) and (iii), and dually of (ii*) and (iii) is straight forward. The implication (iii) $\Longrightarrow$ (i) follows from the fact that $\operatorname{Ext}^{1}\left(M_{i}, M_{j}\right)=0$ in case $M_{i}, M_{j}$ are regular and belong to different Auslander-Reiten components. The converse implication (i) $\Longrightarrow$ (iii) follows from the fact that $\operatorname{Ext}^{1}\left(M_{i}, M_{j}\right) \neq 0$ in case $M_{i}, M_{j}$ are nonzero regular and belong to the same Auslander-Reiten component. In order to see the implication (iii) $\Longrightarrow$ (iv), one should be aware that for $M=\bigoplus_{i=1}^{n} M_{i}$, with indecomposable modules $M_{i}$ belonging to pairwise different tubes, one has $\operatorname{End}(M)=\prod_{i} \operatorname{End}\left(M_{i}\right)$ and that $\operatorname{End}(R)$ is commutative for any indecomposable regular module $R$. Conversely, in order to show the implication (iv) $\Longrightarrow$ (iii), assume that $M$ is regular and assume that $M=M^{\prime} \oplus M^{\prime \prime} \oplus M^{\prime \prime}$ with $M^{\prime}, M^{\prime \prime}$ non-zero modules belonging to some tube. Then $\operatorname{Hom}\left(M^{\prime}, M^{\prime \prime}\right) \neq 0$, thus there is a non-zero homomorphism $\phi: M^{\prime} \rightarrow M^{\prime \prime}$ and we may consider this as an endomorphism of $M$ by setting $\phi$ to be zero on $M^{\prime \prime} \oplus M^{\prime \prime \prime}$. Let $e^{\prime}: M \rightarrow M$ be the projection of $M$ to $M^{\prime}$ with kernel $M^{\prime \prime} \oplus M^{\prime \prime \prime}$. Then $\phi e^{\prime}=\phi \neq 0$, whereas $e^{\prime} \phi=0$. This shows that $\operatorname{End}(M)$ is not commutative.

A regular Kronecker-module $M$ will be said to be strongly regular provided the equivalent conditions of the Lemma are satisfied. Let $\mathcal{R}(i)$ be the set of isomorphism classes of strongly regular modules of length $i$. Note that $\mathcal{R}(i)$ is empty in case $i$ is odd or negative, and $\mathcal{R}(0)$ has just one element, namely the isomorphism class of the zero module.

As we have mentioned, we want to see in which way families of modules may be present in ${ }^{C}[\rightarrow Y\rangle^{1}$, with $C$ indecomposable preprojective, and $Y$ indecomposable preinjective. Here is the description of these sets.

Proposition 14.5 Let $\Lambda$ be the Kronecker algebra, $C$ indecomposable preprojective, $Y$ indecomposable preinjective.

If $C=P(S)$ for some simple module $S$, then ${ }^{C}[\rightarrow Y\rangle^{1}$ may be identified with the set of inclusion maps $X \rightarrow Y$ such that the socle of $Y / X$ is equal to $S$.

If $C=\tau^{-} K$ for some indecomposable module $K$, then ${ }^{C}[\rightarrow Y\rangle^{1}$ consists of the right equivalence classes of surjective maps $X \rightarrow Y$ with kernel $K$.

Proof See Corollary 3.7 and Corollary 7.3.

Given a morphism $f: X \rightarrow Y$, let $\sigma(f)=[X]$, the isomorphism class of the source $X$ of $f$. We study the function $\sigma$ defined on ${ }^{C}[\rightarrow Y\rangle^{1}$ with values in the set of isomorphism classes of modules. The main result of this section is the following description of ${ }^{C}[\rightarrow Y\rangle^{1}$ :

Proposition 14.6 Let $\Lambda$ be the Kronecker algebra, $C$ indecomposable preprojective, $Y$ indecomposable preinjective. Then $\sigma$ is a bijection

$$
\sigma:{ }^{C}[\rightarrow Y\rangle^{1} \longrightarrow \mathcal{R}(i) \quad \text { with } \quad i=|C|+|Y|-4
$$

For the proof, we need some preliminary considerations. 
Lemma 14.7 Let $\Lambda$ be the Kronecker algebra, let $0 \rightarrow U \rightarrow X \rightarrow Y \rightarrow 0$ be a non-split exact sequence with $Y$ indecomposable preinjective, $U$ preprojective. Then no indecomposable direct summand of $X$ is preinjective.

Proof Let $X=X^{\prime} \oplus X^{\prime \prime}$ with $X^{\prime}$ indecomposable. Assume that $X^{\prime}$ is preinjective. Denote the map $X \rightarrow Y$ by $f$ and let $f^{\prime}$ be its restriction to $X^{\prime}$. Then $f^{\prime} \neq 0$, since otherwise $X^{\prime}$ is a direct summand of the kernel of $f$, thus equal to $K$, so that the sequence splits. Non-zero maps between indecomposable preinjective modules are surjective, thus $f^{\prime}$ is surjective. Of course, $f^{\prime}$ is not an isomorphism, since otherwise the sequence would split. Let $U^{\prime}$ be the kernel of $f^{\prime}$. As we see, $U^{\prime} \neq 0$. We have the following commutative diagram with exact rows:

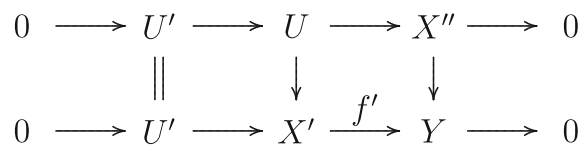

Now $U^{\prime}$ is a submodule of $U$, thus it is preprojective. Since $U^{\prime} \neq 0$, we must have $\delta\left(U^{\prime}\right) \leq-1$, where $\delta$ denotes the defect. It follows that $\delta\left(X^{\prime}\right)=\delta\left(U^{\prime}\right)+\delta(Y) \leq 0$, since $\delta(Y)=1$. This contradicts our assumption that $X^{\prime}$ is preinjective.

Lemma 14.8 Let $X$ be regular, $Y$ indecomposable preinjective. Then the following conditions are equivalent:

(i) $X$ is strongly regular,

(ii) There exists $f: X \rightarrow Y$ such that the kernel of $f$ does not contain a simple regular submodule.

Any map $f: X \rightarrow Y$ with no simple regular submodule in its kernel is a monomorphism or an epimorphism. If $f: X \rightarrow Y$ and $f^{\prime}: X^{\prime} \rightarrow Y$ are maps with no simple regular submodule in the kernel, and $X, X^{\prime}$ are isomorphic, then $f, f^{\prime}$ are right equivalent.

Proof First, we show that (ii) implies (i). Assume that there is $f: X \rightarrow Y$ such that the kernel of $f$ does not contain a simple regular submodule of $X$. In order to show that $X$ is strongly regular, we show that its regular socle is multiplicity-free. Assume, for the contrary, that $X$ has a submodule $U$ such that $U=R \oplus R$ with $R$ simple regular. Let $f_{1}, f_{2}$ be the restrictions of $f$ to $R \oplus 0$ or $0 \oplus R$, respectively. Since no simple regular submodule of $X$ is contained in the kernel of $f$, we see that both $f_{1}, f_{2}$ are non-zero maps. According to Lemma 14.2, there is a map $h: R \rightarrow R$ such that $f_{1}=f_{2} h$. But then $R^{\prime}=\{(-h(x), x) \mid x \in R\}$ belongs to the kernel of $f$. Of course, $R^{\prime}$ is isomorphic to $R$, thus $R^{\prime}$ is a simple regular submodule of $X$ which belongs to the kernel of $f$, a contradiction.

Conversely, assume that $X=\bigoplus_{i} R_{i}\left[t_{i}\right]$ is a strongly regular module, with pairwise different simple regular modules $R_{i}$. According to Lemma 14.2, there is a map $\left.f_{i}: R_{[} t_{i}\right] \rightarrow Y$ such that the restriction of $f_{i}$ to $R_{i}$ is non-zero. Since the simple regular submodules $R_{i}$ are pairwise non-isomorphic, these are the only simple regular submodules of $X$, thus no simple regular submodule of $X$ lies in the kernel of $f=\left(f_{i}\right)_{i}: X \rightarrow Y$, 
Now assume that $f: X \rightarrow Y$ is a map such that the kernel of $f$ does not contain a simple regular submodule. Assume that $f$ is not an epimorphism. The image of $f$ is a factor module of $X$, thus the direct sum of a regular and a preinjective module. But $Y$ has no non-zero proper submodule which is preinjective. Thus, the image of $f$ is regular. The kernel of a map between regular modules is regular, thus either a monomorphism, or it contains a simple regular submodule. Since the latter is not possible, we see that $f$ has to be a monomorphism.

Finally, assume that $f: X \rightarrow Y$ and $f^{\prime}: X^{\prime} \rightarrow Y$ are maps with no simple regular submodule in the kernel. Let $g: X \rightarrow X^{\prime}$ be an isomorphism. Write $X=\bigoplus R_{i}\left[t_{i}\right]$ with pairwise non-isomorphic simple regular modules $R_{i}$. Let $f_{i}$ and $f_{i}^{\prime}$ be the restriction of $f$ and $f^{\prime} g$, respectively, to $R_{i}\left[t_{i}\right]$. Since $R_{i}$ is not in the kernel of $f$, the restriction of $f_{i}$ to $R_{i}$ is non-zero. According to Lemma 14.2, there is a map $h_{i}: R_{i} \rightarrow R_{i}$ such that $f_{i}=f_{i}^{\prime} h_{i}$. But also the restriction of $f_{i}^{\prime}$ to $R_{i}$ is non-zero, thus $R_{i}$ is not in the kernel of $h_{i}$ and therefore $h_{i}$ is an automorphism. Let $h=\left(h_{i}\right)_{i}: X \rightarrow X$. This is an automorphism of $X$ with $f=f^{\prime} g h$. Since $f=f^{\prime} g h$ and $g h$ is an isomorphism, we see that $f, f^{\prime}$ are right equivalent.

Proposition 14.9 Let $Y$ be indecomposable preinjective. The maximal submodules of $Y$ are pairwise non-isomorphic and these are, up to isomorphism, all the strongly regular modules of length $|Y|-1$.

The kernels of the non-zero maps $Y \rightarrow Q_{1}$ are pairwise non-isomorphic and these are, up to isomorphism, all the strongly regular modules of length $|Y|-3$.

Proof Let $X$ be a maximal submodule of $Y$. Then $Y / X$ is simple injective, thus $\delta(X)=\delta(Y)-\delta(Y / X)=0$. Since $Y$ has no proper non-zero preinjective submodule, we see that $X$ has to be regular. According to Lemma 14.8, the inclusion map $X \rightarrow Y$ shows that $X$ is even strongly regular, and of course of length $|Y|-1$. Conversely, if $X$ is strongly regular and of length $|Y|-1$, Lemma 14.8 yields a monomorphism $X \rightarrow Y$. Now assume that two maximal submodules $X, X^{\prime}$ are isomorphic, let $f: X \rightarrow Y$ and $f^{\prime}: X^{\prime} \rightarrow Y$ be the inclusion maps. Then, according to Lemma 14.8, $f, f^{\prime}$ are right equivalent, thus $X=X^{\prime}$.

In the same way, we consider the kernels $X$ of the non-zero maps $Y \rightarrow Q_{1}$. Clearly, all non-zero maps $Y \rightarrow Q_{1}$ are surjective, thus $\delta(X)=0$ and again, $X$ has to be regular, and according to Lemma 14.8 even strongly regular, of course of length $|Y|-3$. Conversely, if $X$ is strongly regular and of length $|Y|-1$, Lemma 14.8 yields a monomorphism $X \rightarrow Y$. The factor module $Y / X$ is of length 3 and has the same composition factors as $Q_{1}$. But the only factor module of $Y$ of length 3 with the same composition factors as $Q_{1}$ is $Q_{1}$ itself, thus $X$ is the kernel of a non-zero map $Y \rightarrow Q_{1}$. Finally, we use again Lemma 14.8 in order to see that isomorphic kernels of non-zero maps $Y \rightarrow Q_{1}$ are actually identical.

Remark It should be stressed that for $C=P_{0}$ and $C=P_{1}$, the inverse $\eta_{C Y}^{-1}$ of the Auslander bijection can be seen very well:

If $M$ is a Kronecker module, we may write $M$ in the form

$$
M=\left(M_{a}, M_{b} ; \alpha: M_{b} \rightarrow M_{a}, \beta: M_{b} \rightarrow M_{a}\right)
$$


where $M_{a}, M_{b}$ are vector spaces and $M_{\alpha}, M_{\beta}$ are linear maps. We can identify $M_{a}$ with $\operatorname{Hom}\left(P_{0}, M\right)$ and $M_{b}$ with $\operatorname{Hom}\left(P_{1}, M\right)$, since $P_{0}=P(a), P_{1}=P(b)$.

Consider the case $C=P_{1}$. Given a maximal submodule $U$ of $\operatorname{Hom}(C, Y)$, we may interpret it as a maximal submodule of $Y_{b}=\operatorname{Hom}(C, Y)$, and we may consider the submodule $\Lambda U$ of $Y$ generated by $U$, let $u: \Lambda U \rightarrow Y$ be the inclusion map. Then $[u: \Lambda U \rightarrow Y\rangle$ belongs to ${ }^{C}[\rightarrow Y\rangle_{1}$ and $\eta_{C V}(u)=U$. This shows that $[u\rangle=\eta_{C Y}^{-1}(U)$.

Similarly, for $C=P_{0}$, starting with a maximal subspace $V_{a}$ of the vector space $Y_{a}=\operatorname{Hom}(C, Y)$, we may construct $V_{b}=Y_{\alpha}^{-1}\left(V_{a}\right) \cap Y_{\beta}^{-1}\left(V_{a}\right)$, then $V=$ $\left(V_{a}, V_{b} ; Y_{\alpha}\left|V_{b}, Y_{\beta}\right| V_{b}\right)$ is a submodule of $Y$, say with inclusion map $v: V \rightarrow Y$. Then $[v: V \rightarrow Y\rangle$ belongs to ${ }^{C}[\rightarrow Y\rangle_{1}$ and $\eta_{C V}(v)=V_{a}$. This shows that $[v\rangle=\eta_{C Y}^{-1}\left(V_{a}\right)$.

Proposition 14.10 Let $\Lambda$ be the Kronecker algebra and $Y$ indecomposable preinjective, $C$ indecomposable preprojective. Let $d=|C|+|Y|-4$. Let $X$ be any module.

There is $f: X \rightarrow Y$ right minimal, right $C$-determined with $|f|_{C}=1$, if any only if the isomorphism class of $X$ belongs to $\mathcal{R}(d)$.

If $f: X \rightarrow Y$ and $f^{\prime}: X^{\prime} \rightarrow Y$ are right minimal, right $C$-determined maps with $|f|_{C}=\left|f^{\prime}\right|_{C}=1$. Then $f, f^{\prime}$ are right equivalent if and only if $X, X^{\prime}$ are isomorphic.

Proof First, consider the case when $C$ is not projective, thus $|C| \geq 5$, therefore $d>|Y|$.

Since $\Lambda$ is hereditary and $C$ has no indecomposable projective direct summand, any right $C$-determined map $f: X \rightarrow Y$ is surjective and its kernel belongs to add $K$, where $K=\tau C$. If we assume that $|f|_{C}=1$, then the kernel of $f$ has to be equal to $K$. It follows that $\delta(X)=\delta(Y)+\delta(K)=0$. Since all indecomposable submodules of $X$ are preprojective or regular, we see that $X$ has to be regular. Of course, its length is just $d$. Note that no simple regular submodule $R$ of $X$ can be contained in the kernel of $f$, since by assumption the kernel of $f$ is $K$, thus preprojective. The Lemma 14.8 shows that $X$ is strongly regular, thus the isomorphism class of $X$ belongs to $\mathcal{R}(d)$.

Conversely, assume that $X$ is a strongly regular module of length $|X|=d$. According to the Lemma 14.8 there exists a morphism $f: X \rightarrow Y$ such that its kernel contains no simple regular module. Since $|X|=d>|Y|$, the map $f$ cannot be a monomorphism, thus it is an epimorphism. Since the kernel of $f$ does not contain a simple regular module, it is the direct sum of indecomposable preprojective modules. Since the defect of the kernel is $\delta(X)-\delta(Y)=-1$, we see that the kernel of $f$ is indecomposable preprojective. The length of the kernel is $|X|-|Y|=|C|-4=|\tau C|$, thus the kernel of $f$ is isomorphic to $\tau C$. Altogether, we have shown: if the isomorphism class of $X$ belongs to $\mathcal{R}(d)$, then there is $f: X \rightarrow Y$ right minimal, right $C$-determined with $|f|_{C}=1$.

Finally, assume that $f: X \rightarrow Y$ and $f^{\prime}: X^{\prime} \rightarrow Y$ are right minimal, right $C$ determined maps with $|f|_{C}=\left|f^{\prime}\right|_{C}=1$. Then $X, X^{\prime}$ are strongly regular. If these maps are right equivalent, then clearly $X, X^{\prime}$ are isomorphic. Conversely, assume that $X, X^{\prime}$ are isomorphic. In order to show that $f, f^{\prime}$ are right equivalent, we may assume that $X^{\prime}=X$. Lemma 14.8 asserts that $f, f^{\prime}$ are right equivalent.

Now assume that $C$ is projective, thus $|C| \leq 3$, therefore $d<|Y|$. In case $C=P_{1}$, we have to consider the submodules $X$ of $Y$ with $Y / X=Q_{0}$, in case $C=P_{0}$, we have to consider the submodules $X$ of $Y$ with $Y / X=Q_{1}$. This has been done in the previous proposition. 
Proof of proposition 14.6 The proposition is an immediate consequence of Proposition 14.5 and Proposition 14.10.

Corollary 14.11 Let $\Lambda$ be the Kronecker algebra, $C$ indecomposable preprojective, $Y$ indecomposable preinjective. Then $\sigma \eta_{C Y}^{-1}$ yields a bijection

$$
\sigma \eta_{C Y}^{-1}: \mathcal{S}_{m} \operatorname{Hom}(C, Y) \longrightarrow \mathcal{R}(i) \quad \text { with } \quad i=|C|+|Y|-4
$$

Note that $\Gamma(C)=k$, thus $\operatorname{Hom}(C, Y)$, considered as a $\Gamma(C)$-module is just a vector space, thus $\mathcal{S}_{m} \operatorname{Hom}(C, Y)$ is the set of the maximal subspaces of a vector space, and therefore a projective space. We see that we obtain a parameterization of the $\operatorname{set} \mathcal{R}(2 i)$ of all strongly regular Kronecker modules of length $2 i$ by the projective space $\mathbb{P}^{i}$.

Remark This parameterization of $\mathcal{R}(d)$ extends the well-known description of the geometric quotient of the "open sheet", when dealing with conjugacy classes of $(n \times n)$ matrices with coefficients in a field, see for example Kraft [23].

Let us mention some details: Let $R=k[T]$ be the polynomial ring in one variable $T$ with coefficients in the field $k$, and let us assume that $k$ is algebraically closed. We consider $R$ as the path algebra of the quiver with one vertex and one loop; in this way, the $R$-modules of dimension $n$ are just pairs $(V, \phi)$, where $V$ is a $k$-space of dimension $n$ and $\phi: V \rightarrow V$ an endomorphism of $V$, or, after choosing a basis of $V$, we just deal with $(n \times n)$-matrices with coefficients in $k$. Isomorphism of $R$ modules translates to equivalence (or conjugacy) of matrices. The assertions of Lemma 14.4 can be reformulated in this context, the properties mentioned there characterize just the cyclic R-modules of finite length. Note that the category of $k[T]$-modules of finite length is equivalent to the full subcategory $\mathcal{R}^{\prime}$ of all regular Kronecker modules without eigenvalue $\infty$ (to be precise: let us denote the two arrows of the Kronecker quiver by $\alpha, \beta$ and let $\mathcal{T}_{\infty}$ be the Auslander-Reiten component which contains the indecomposable regular representation $M$ with $M_{\alpha}=0$. The subcategory $\mathcal{R}^{\prime}$ consists of all regular representations with no indecomposable direct summand in $\mathcal{T}_{\infty}$, or equivalently, it is the full subcategory of all representations $M$ such that $M_{\alpha}$ is bijective. Under the equivalence of the category of finite-dimensional $k[T]$-modules and $\mathcal{R}^{\prime}$ $k[T]$-module $N$ corresponds to the representation $M$ such that $M_{\alpha}: N \rightarrow N$ is the identity map, and $M_{\beta}: N \rightarrow N$ the multiplication by $T$ ). A representation $M$ in $\mathcal{R}^{\prime}$ is strongly regular if and only if $M$ corresponds under this equivalence to a cyclic $k[T]-$ module. Thus, one may be tempted to call the strongly regular Kronecker modules "cyclic" modules, but this would be in conflict with standard terminology.

Remark "Modules determined by morphisms". A bijection of two sets can always be read in two different directions. This survey is concerned with the Auslander bijection

$$
\eta_{C Y}:{ }^{C}[\rightarrow Y\rangle \longrightarrow \mathcal{S} \operatorname{Hom}(C, Y),
$$

In the previous sections, the focus was going from left to right: Any right minimal morphism $f \in{ }^{C}[\rightarrow Y\rangle$ yields under $\eta_{C Y}$ a submodule of $\operatorname{Hom}(C, Y)$ and is uniquely 
determined by this submodule, this is the philosophy of saying that morphisms as elements of $[\rightarrow Y\rangle$ ) are determined by modules.

The considerations in the present section point into the reverse direction: we use sets of morphisms as convenient indices for parameterizing isomorphism classes of modules. We recall from Sect. 7 that the restriction of $\eta_{C Y}$ furnishes a bijection

$$
\eta_{C Y}:{ }^{C}[\rightarrow Y\rangle^{1} \longrightarrow \mathcal{S}_{m} \operatorname{Hom}(C, Y),
$$

thus we can use the right hand set in order to parametrize the left hand set.

Our interest lies in the special case where maps $f: M \rightarrow Y$ and $f^{\prime}: M^{\prime} \rightarrow Y$ of right $C$-length 1 are right equivalent only in case $M$ and $M^{\prime}$ are isomorphic. In this case, the maximal submodule $\eta_{C Y}(f: M \rightarrow Y)$ (thus a set of morphisms) uniquely determines the module $M$. In addition, we will assume that $C$ is a brick, or at least that $C$ is indecomposable and $\operatorname{rad} \operatorname{End}(C)$ annihilates $\operatorname{Hom}(C, Y)$. In this case, $\mathcal{S}_{m} \operatorname{Hom}(C, Y)$ is a projective space (namely the projective $d$-space over the division ring $\operatorname{End}(C) / \operatorname{rad} \operatorname{End}(C)$, provided $\operatorname{Hom}(C, Y)$ is a module of length $d+1)$.

Let us determine $\eta_{C Y}^{-1}(0)$ for $C=P_{i}, Y=Q_{j}$ with $i, j \in \mathbb{N}_{0}$. Note that

$$
\operatorname{dim} \operatorname{Hom}\left(P_{i-1}, Q_{j}\right)=i+j-1,
$$

where $P_{-1}=0$. Thus, the universal map from add $P_{i-1}$ to $Q_{j}$ is of the form $P_{i-1}^{i+j-1} \rightarrow$ $Q_{j}$ (for $j=0$, it is a map of the form $P_{i-1}^{i-1} \rightarrow Q_{0}$ ).

Proposition 14.12 Let $\Lambda$ be the Kronecker algebra. Let $C=P_{i}, Y=Q_{j}$ with $i, j \in \mathbb{N}_{0}$. Let $f: P_{i-1}^{i+j-1} \rightarrow Q_{j}$ be the universal map from add $P_{i-1}$ to $Q_{j}$. Then

$$
\eta_{C Y}^{-1}(0)=\left[f: P_{i-1}^{i+j-1} \rightarrow Q_{j}\right\rangle
$$

Proof For $i=0$, the intersection of the kernels of the maps $Q_{j} \rightarrow Q_{1}$ is zero. For $i=1$, the module $P_{0}=P_{i-1}$ is simple projective, thus, for $j \geq 1$, the universal map $f: P_{0}^{j} \rightarrow Q_{j}$ is just the embedding of the socle of $Q_{j}$ into $Q_{j}$ and the socle is the intersection of the kernels of the maps $Q_{j} \rightarrow Q_{0}$. For $i=1$ and $j=0$, the intersection of the kernels of the maps $Q_{j} \rightarrow Q_{0}$ is zero, but also $P_{i-1}^{i+j-1}$ is zero.

Assume now that $i \geq 2$. We have

$$
\operatorname{dim} \operatorname{Ext}^{1}\left(Q_{j}, P_{i-2}\right)=\operatorname{dim} \operatorname{Hom}\left(P_{i}, Q_{j}\right)=\operatorname{dim} \operatorname{Hom}\left(P_{i+j}, Q_{0}\right)=i+j,
$$

thus the universal extension of $Q_{j}$ from below using copies of $P_{i-2}$ looks as follows

$$
0 \rightarrow P_{i-2}^{i+j} \rightarrow X \stackrel{f}{\rightarrow} Q_{j} \rightarrow 0
$$

with a module $X$ such that $\operatorname{Ext}^{1}(X, X)=0$. The dimension vector of $X$ is

$$
\operatorname{dim} X=(i+j) \operatorname{dim} P_{i-2}+\operatorname{dim} Q_{j}=(i+j-1) \operatorname{dim} P_{i-1} .
$$


Since also $\operatorname{Ext}^{1}\left(P_{i-1}, P_{i-1}\right)=0$, it follows that $X=P_{i-1}^{i+j-1}$. Since $f$ is right minimal and $\operatorname{dim} \operatorname{Hom}\left(P_{i-1}, Q_{j}\right)=i+j-1$, we see that $f$ has to be the universal map from add $P_{i-1}$ to $Q_{j}$.

Example 11 We consider the special case of the Kronecker modules $C=P_{4}, Y=Q_{0}$. Note that $\operatorname{dim} Y=(0,1)$ and $\operatorname{dim} P_{4}=(5,4)$, thus $\tau C=P_{2}$ has dimension vector $(3,2)$ and $\operatorname{Hom}(\tau C, Y)=2$. Here is a sketch of ${ }^{C}[\rightarrow Y\rangle$. In any layer ${ }^{C}[\rightarrow Y\rangle_{t}$ with $1 \leq t \leq 3$, we indicate the elements $[f: X \rightarrow Y\rangle$ in the form $P_{2}^{t} \rightarrow \operatorname{dim} X \rightarrow Y$. The map in the layer $t=0$ has been described in Proposition 14.12.

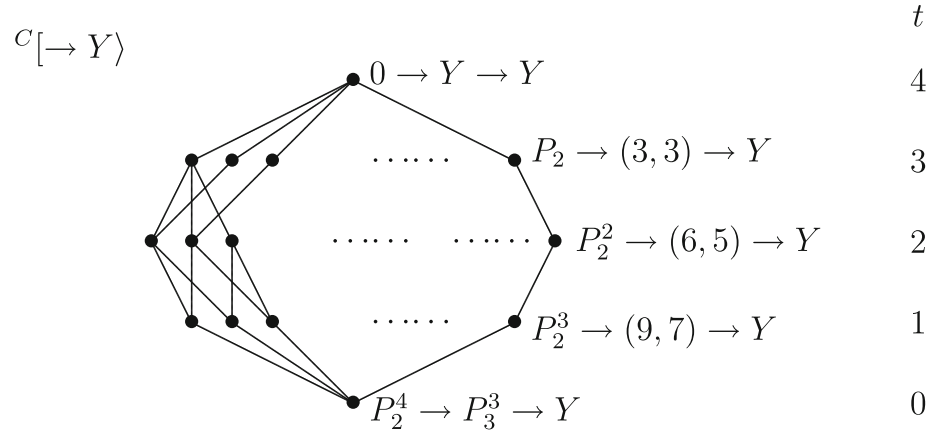

Let us exhibit some of the short exact sequences $P_{1}^{t} \rightarrow X \rightarrow Y$.

For $(3,3)$, the module $X$ must be a strongly regular module, thus, there are three different kinds: direct sums of three pairwise non-isomorphic indecomposable modules of length 2, direct sums of an indecomposable module $M$ of length 4 and an indecomposable module $M^{\prime}$ of length 2 such that $\operatorname{Hom}\left(M, M^{\prime}\right)=0$, and finally indecomposable modules of length 6 .

For $(6,5)$ and $(9,7)$, we deal with direct sums of preprojective and regular modules. We consider the case $\operatorname{dim} X=(6,5)$, thus $t=2$, in detail. Let $\left.X=X_{a}, X_{b} ; X_{\alpha}, X_{\beta}\right)$ be a Kronecker module with a submodule $U$ isomorphic to $P_{2}^{2}$ such that $X / U$ is isomorphic to $Q_{0}$.

We start with a basis of $U_{b}$ and add an element $x$ in order to obtain a basis of $X_{b}$, thus $X_{a}=U_{a}$, and $X_{b}=U_{b} \oplus\langle x\rangle$. What we have to describe are the elements $\alpha(x)$ and $\beta(x)$ in $U_{a}$, and we have to provide a decomposition of $X$ into indecomposables. In this way, we also will see that the map $X \rightarrow Q_{0}$ with kernel $U$ is right minimal.

Since $U$ is isomorphic to $P_{2}^{2}$, we can exhibit $U$ as follows: $U_{a}$ has a basis $v_{0}, \ldots, v_{5}$ and $U_{b}$ has a basis $u_{1}, u_{2}, u_{4}, u_{5}$ and $\alpha\left(u_{i}\right)=v_{-1}$ and $\beta\left(u_{i}\right)=v_{i}$. In order to describe $X$, we have to discuss possible values for $\alpha(x)$ and $\beta(x)$. Four different cases will be of interest. In the first three cases, let $\alpha(x)=v_{2}$.

(1) If we define $\beta(x)=v_{3}$, then clearly $X=P_{5}$.

(2) If we define $\beta(x)=v_{4}$, then we get a decomposition of $X$ as follows: The elements $u_{1}, u_{2}, x, u_{5}, v_{0}, v_{1}, v_{2}, v_{4}, v_{5}$ yield a submodule of the form $P_{4}$, the elements $x-u_{4}, v_{2}-v_{3}$ also yield a submodule (since $\beta\left(x-u_{4}\right)=0$ ). These two submodules provide a direct decomposition.

(3) If we define $\beta(x)=v_{5}$, then we see that the elements $u_{1}, u_{2}, x ; v_{0}, v_{1}, v_{2}, v_{5}$ yield a submodule of the form $P_{3}$. The elements $u_{2}-u_{4}, x-u_{5}, v_{1}-v_{3}, v_{2}-v_{5}$ 
yield a 4-dimensional indecomposable submodule, and we obtain in this way a direct decomposition.

(4) Finally, let $\alpha(x)=v_{1}, \beta(x)=v_{4}$. Then we get a submodule of $X$ with basis $u_{1}, x, u_{5}, v_{0}, v_{1}, v_{4}, v_{5}$ which is of the form $P_{3}$ as well as two indecomposable submodules of length 2 , namely with basis $x-u_{1}, v_{4}-v_{2}$ and with basis $x-u_{4}, u_{1}-u_{3}$.

In this way, we obtain short exact sequences

$$
0 \rightarrow P_{2}^{2} \rightarrow X \rightarrow Q_{0} \rightarrow 0
$$

such that $X$ is of the form $P_{5}$, of the form $P_{4} \oplus R$, of the form $P_{3} \oplus R^{\prime \prime}$, or finally of the form $P_{3} \oplus R \oplus R^{\prime}$, where $R, R^{\prime}$ are both regular of length 2 , and $R^{\prime \prime}$ is regular of length 4 .

\section{Lattices of height at most 2}

The height of $\mathcal{S} \operatorname{Hom}(C, Y)$ is the length of the $\Gamma(C)$-module $\operatorname{Hom}(C, Y)$. If $\Lambda$ is a $k$-algebra, $k$ is algebraically closed, and $C$ is multiplicity-free (what we can assume), then the height of $\mathcal{S} \operatorname{Hom}(C, Y)$ is the $k$-dimension of $\operatorname{Hom}(C, Y)$.

Our main interest will concern the height 2 lattices which are not distributive, since this is the first time that one may encounter infinite families. Here is a discussion of the lattices of height at most 2 , in general.

Height $\mathbf{0}$. The lattice $\mathcal{S} M$ has height 0 if and only if $M=0$. Thus, to say that $\mathcal{S} \operatorname{Hom}(C, Y)$ has height zero means that $\operatorname{Hom}(C, Y)=0$.

Height 1. The lattice $\mathcal{S} M$ has height 1 if and only if $M$ is a simple module. Thus, in our case $M=\operatorname{Hom}(C, Y)$, we deal with a simple $\Gamma(C)$-module. Note that $\operatorname{Hom}(C, Y)$ is a simple $\Gamma(C)$-module if any only if there is a right minimal, right $C$-determined map $f: X \rightarrow Y$ which is not an isomorphism, such that for any right minimal, right $C$-determined map $f^{\prime}: X^{\prime} \rightarrow Y$ which is not an isomorphism, there is an isomorphism $h: X \rightarrow X^{\prime}$ such that $f=f^{\prime} h$.

Three cases should be noted:

(1) $f$ may be an epimorphism. For example, take the path algebra of the quiver of type $\mathbb{A}_{2}$ as exhibited in example 1 . Let $C=Y=S(b)$ (thus $\tau C=S(a)$ ). Then the epimorphism $f: P(b) \rightarrow S(b)=Y$ is, up to isomorphism, the only right minimal, right $C$-determined map ending in $Y$ which is not an isomorphism.

(2) $f$ may be a monomorphism. To obtain an example, take again the path algebra of the quiver of type $\mathbb{A}_{2}$. Let $C=S(a)$ and $Y=P(b)$, thus now $C$ is projective. The monomorphism $f: S(a) \rightarrow P(b)=Y$ is, up to isomorphism, the only right minimal, right $C$-determined map ending in $Y$ which is not an isomorphism.

(3) $f$ is neither epi nor mono. As an example, take the radical square zero algebra with the linearly oriented quiver of type $\mathbb{A}_{3}$, see example 6 . Let $Y=P(c)$ and $C=S(b)$ (thus $\tau C=S(a)$ ). The non-zero map $f: P(b) \rightarrow P(c)$ is, up to right equivalence, the only right minimal, right $C$-determined map ending in $Y$ which is not an isomorphism. 
Height 2. A lattice of height 2 may be either a chain (thus of the form $\mathbb{I}(2)$ ) or not. If the lattice is not a chain, the lattice still may be distributive (case III) or not (case IV). The submodule lattices $\mathcal{S} M$ of type III and IV occur for a semisimple module $M$ of length 2; in case $M$ is the direct sum of two non-isomorphic simple modules, we deal with case III, otherwise $M$ is the direct sum of two isomorphic simple modules and then we deal with case IV. Since the lattices we are interested are submodule lattices (here of a module of length 2), we distinguish also in the case of a serial module $M$ of length 2 , whether the two composition factors are isomorphic (case I) or not (case II).

Altogether, we see that for a lattice $\mathcal{S} \operatorname{Hom}(C, Y)$ of height 2, there are the following four cases:

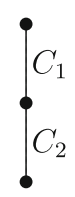

type I

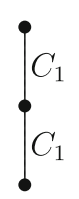

II

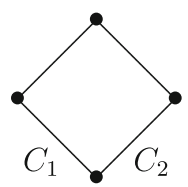

III

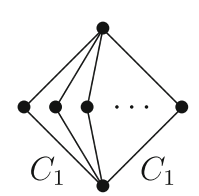

IV

here, the labels $C_{1}, C_{2}$ concern the type of the corresponding pair of neighbors: $C_{1}, C_{2}$ are non-isomorphic indecomposable modules.

Type I. Example 12 Take the linearly oriented quiver of type $\mathbb{A}_{3}$ as discussed in Examples 5. Let $Y=S(3)$ and $C=Q(2) \oplus S(3)$. Then $\Gamma(C)$ is the path algebra of the quiver of type $\mathbb{A}_{2}$ and $\operatorname{Hom}(C, Y)$ is the indecomposable $\Gamma(C)$ module of length 2. The lattices ${ }^{C}[\rightarrow Y\rangle$ and $\mathcal{S} \operatorname{Hom}(C, Y)$ look as follows:
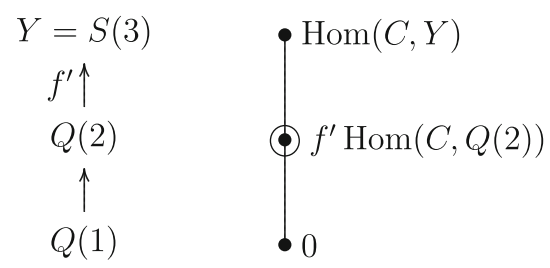

Type II. In the last section, we have seen such examples for the Kronecker algebra, namely: if $C, Y$ are modules with $C$ indecomposable, such that ${ }^{C}[\rightarrow Y\rangle$ is of the form $\mathbb{I}(2)$, then we must be in type II.

Another example has been presented already in Sect. 7 when dealing with Riedtmann-Zwara degenerations, namely example 9. There, we have chosen (nonprojective) indecomposable modules $C$ and $Y$ such that $\operatorname{Hom}(C, Y)$ was a cyclic module of length 2 . There, an additional module $Y$ was considered with an epimorphism $M^{\prime} \rightarrow Y$ such that the composition $M \rightarrow M^{\prime} \rightarrow Y$ is non-zero. Note that this procedure fits into the consideration of families $\mathcal{M}=\left\{M_{i} \mid i \in I\right\}$ of modules which is based on dealing with fixed morphisms $f_{i}: M_{i} \rightarrow Y$ for some module $Y$.

Type III. Example 13 Take the quiver of type $\mathbb{A}_{3}$ with two sinks: 


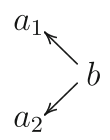

and let $\Lambda$ be its path algebra. Here is the Auslander-Reiten quiver of $\Lambda$ :

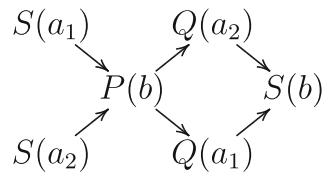

Let $Y=S(b)$ and $C=Q\left(a_{1}\right) \oplus Q\left(a_{2}\right)$. As usual, let us show both ${ }^{C}[\rightarrow Y\rangle$ as well as $\mathcal{S} \operatorname{Hom}(C, Y)$ :
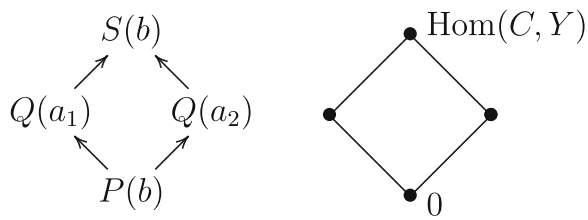

Example 14 Here is a second example of type III. In contrast to the previous example, here the two incomparable right minimal maps $f: X \rightarrow Y$ and $f^{\prime}: X^{\prime} \rightarrow Y$ have the property that the modules $X$ and $X^{\prime}$ are isomorphic. We consider the Kronecker algebra (see Sect. 11). Let $Y=S(b)$ and $C=R \oplus R^{\prime}$, where $R, R^{\prime}$ are non-isomorphic regular modules of length 2 . We may consider $R, R^{\prime}$ as submodules of $Q(a)$ and we denote by $f: Q(a) \rightarrow S(b)$ the projection with kernel $R$, by $f^{\prime}: Q(a) \rightarrow S(b)$ the projection with kernel $R^{\prime}$. The pullback of $f$ and $f^{\prime}$ is the preinjective module $Q_{2}$ (with dimension vector $(2,3)$ ).
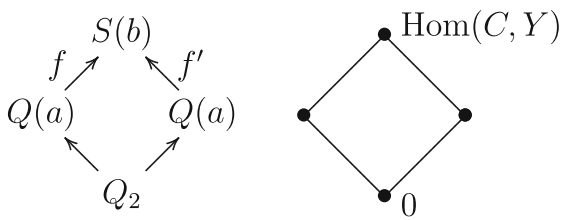

Type IV. Let $C, Y$ be a pair of modules such that ${ }^{C}[\rightarrow Y\rangle$ is of type IV. As we will see, and this is our main concern, the behaviour of the modules present in ${ }^{C}[\rightarrow Y\rangle^{1}$ may be quite different.

Example 15 where the modules present in ${ }^{C}[\rightarrow Y\rangle^{1}$ are all isomorphic. As we have seen in Sect. 11, there are many such examples for the Kronecker algebra, thus let $\Lambda$ be the Kronecker algebra, let $P_{0}, P_{1}, P_{2}, \ldots$ be the indecomposable preprojective modules, and $Q_{0}, Q_{1}, Q_{2}, \ldots$ the indecomposable preinjective modules, with both $P_{i}$ and $Q_{i}$ being of length $2 i+1$.

First, let $C=P_{i}$ and $Y=P_{i+1}$, for some $i \geq 0$, thus $\operatorname{dim} \operatorname{Hom}(C, Y)=2$ and therefore $\mathcal{S} \operatorname{Hom}(C, Y)$ is of the form IV.

If $i=0$, then we deal with the lattice of submodules $U$ of $P_{1}$ such that the socle of $P_{1} / U$ is generated by $P_{0}$. Such a submodule is either 0 or simple (thus of the form 
$\left.P_{0}\right)$ or equal to $P_{1}$ :

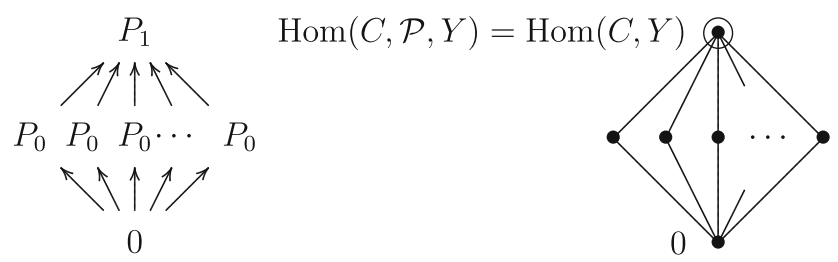

If $i=1$, then we deal with the lattice of all submodules $U$ of $P_{2}$ which contain the socle of $P_{2}$ (these are just the submodules $U$ of $P_{2}$ such that the socle of $P_{2} / U$ is generated by $\left.P_{1}\right)$. Note that such a submodule is either the socle itself, thus isomorphic to $P_{0}{ }^{3}$, or of the form $N=P_{0} \oplus P_{1}$, or $P_{2}$ itself.

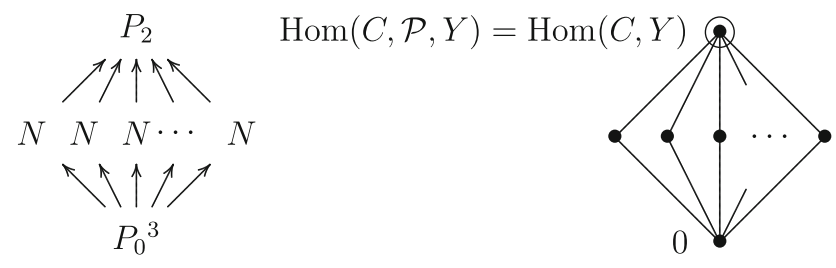

$$
\text { with } N=P_{0} \oplus P_{1}
$$

Whereas for the cases $i=0,1$ all the maps shown in the lattice ${ }^{C}[\rightarrow Y\rangle$ are inclusion maps, the maps exhibited in ${ }^{C}[\rightarrow Y\rangle$ for $i \geq 2$ are all epimorphisms, see Proposition 6.6 and Lemma 6.8.

So let us assume that $i \geq 2$. A map $f: X \rightarrow Y$ is right $P_{i}$-determined if and only if its kernel is a direct sum of copies of $P_{i-2}$. Since $\operatorname{Ext}^{1}\left(P_{i+1}, P_{i-2}\right)=$ $D \operatorname{Hom}\left(P_{i}, P_{i+1}\right)$ is a two-dimensional vector space, we see that we deal with short exact sequences of the form

$$
\begin{gathered}
0 \rightarrow P_{i-2} \rightarrow N \rightarrow P_{i+1} \rightarrow 0 \\
0 \rightarrow P_{i-2}^{2} \rightarrow M \rightarrow P_{i+1} \rightarrow 0
\end{gathered}
$$

such that the maps $N \rightarrow P_{i+1}$ and $M \rightarrow P_{i+1}$ are right minimal. It follows easily that all these modules $N$ have to be of the form $P_{i-1} \oplus P_{i}$, and $M$ has to be of the form $P_{i-1}{ }^{3}$. Thus, the lattice ${ }^{C}[\rightarrow Y\rangle$ looks as follows:
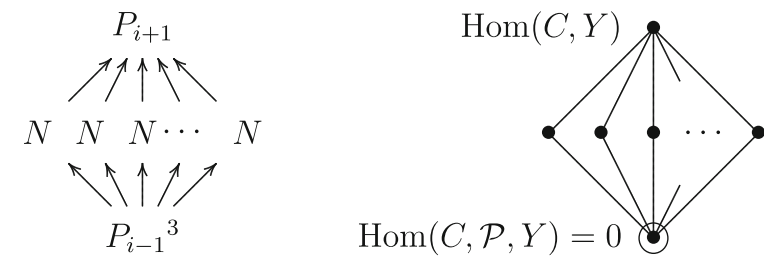

with $N=P_{i-1} \oplus P_{i}$

Let us describe the right minimal maps $N=P_{1} \oplus P_{2} \rightarrow P_{3}$ in detail. We have $\operatorname{dim} \operatorname{Hom}\left(P_{1} \oplus P_{2}, P_{3}\right)=3$, but actually only the homomorphisms $P_{2} \rightarrow P_{3}$ matter and $\operatorname{Hom}\left(P_{2}, P_{3}\right)=2$. Why only the homomorphisms $P_{2} \rightarrow P_{3}$ matter? We need an epimorphism $f: P_{1} \oplus P_{2} \rightarrow P_{3}$, write it as $f=\left[f_{1}, f_{2}\right]$ with $f_{1}: P_{1} \rightarrow P_{3}$ and 
$f_{2}: P_{2} \rightarrow P_{3}$. In order that $f$ is an epimorphism, the following two conditions have to be satisfied:

(1) The restriction $f_{2}$ of $f$ to $P_{2}$ has to be non-zero.

(2) The image $f_{1}\left(P_{1}\right)$ is not contained in $f_{2}\left(P_{2}\right)$, or, equivalently (since $P_{1}$ is projective) $f_{1}$ does not factor through $f_{2}$.

If two such maps $\left[f_{1}, f_{2}\right]$ and $\left[f_{1}^{\prime}, f_{2}^{\prime}\right]$ are given with both $f_{2}$ and $f_{2}^{\prime}$ non-zero, and $f_{1}\left(P_{1}\right) \not \subset f_{2}\left(P_{2}\right)$ as well as $f_{1}^{\prime}\left(P_{1}\right) \not \subset f_{2}^{\prime}\left(P_{2}\right)$, then $\left[f_{1}, f_{2}\right]$ is right equivalent to $\left[f_{1}^{\prime}, f_{2}^{\prime}\right]$ if and only if $f_{2}$ is right equivalent to $f_{2}^{\prime}$, if and only if there is a scalar $c \in k^{*}$ such that $f_{2}^{\prime}=c f_{2}$.

It follows that the existence of the one-parameter family of right minimal maps $N \rightarrow P_{3}$ comes from the fact that $\operatorname{dim} \operatorname{Hom}\left(P_{2}, P_{3}\right)=2$.

Observe that the lattices ${ }^{C}[\rightarrow Y\rangle$ for $C=P_{i}, Y=P_{i+1}$ and all $i \geq 0$ have the same form, provided we set $P_{-1}=0$,

Example 16 where the modules in ${ }^{C}[\rightarrow Y\rangle^{1}$ are pairwise non-isomorphic. Again, we deal with the Kronecker algebra. Let $Y=Q_{0}$ and $C=P_{2}$, thus $\operatorname{dim} \operatorname{Hom}(C, Y)=$ 2 and $K=\tau C=P_{0}$. The right minimal right $C$-determined morphisms ending in $Y$ are epimorphisms with kernel in add $K$. Here are the lattices in question:
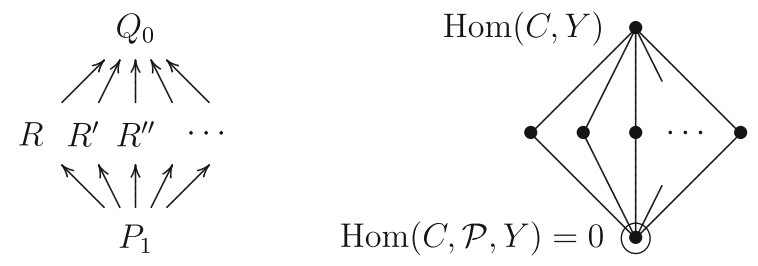

with pairwise non-isomorphic indecomposable representations $R, R^{\prime}, R^{\prime \prime}, \ldots$ of length 2.

\section{Example 17 where the modules in ${ }^{C}[\rightarrow Y\rangle^{1}$ belong to a finite number of isomor- phism classes with a fixed dimension vector.}

Take the 3-subspace quiver as considered in example 9. Let $Y=Q(a)$, and $C$ the maximal indecomposable module, thus $C=\tau Q(a)$ and $\operatorname{dim} \operatorname{Hom}(C, Y)=2$. Then $\mathcal{S} \operatorname{Hom}(C, Y)$ is the non-distributive lattice of height 2 , and the elements of height 1 form a $\mathbb{P}_{1}$-family.

This $\mathbb{P}_{1}$-family in $\mathcal{S} \operatorname{Hom}(C, Y)$ contains three special elements, namely the three subspaces which are generated by the composition of irreducible maps $C \rightarrow \tau^{-} P\left(b_{i}\right)$ and $\tau^{-} P\left(b_{i}\right) \rightarrow Y$, for $1 \leq i \leq 3$. The remaining elements of the $\mathbb{P}_{1}$-family are generated by the surjective maps $C \rightarrow Y$.

Correspondingly, in ${ }^{C}[\rightarrow Y\rangle_{1}$, there are the right equivalence classes $\left[f_{i}\right\rangle$ of surjective maps $f_{i}: M(i) \rightarrow Y$, where $M(i)=P\left(b_{i}\right) \oplus \tau^{-} P\left(b_{i}\right)$ for $1 \leq i \leq 3$; the remaining elements of the $\mathbb{P}_{1}$-family are the right equivalence classes of the surjective maps $C \rightarrow Y$. Note that all the modules $M(1), M(2), M(3)$ as well as $C$ have the same dimension vector, namely $2 \frac{1}{1}$.

The zero element of the lattice ${ }^{C}[\rightarrow Y\rangle$ is the projective cover $P(Y) \rightarrow Y$. In the submodule lattice $\mathcal{S} \operatorname{Hom}(C, Y)$, the zero element is $\operatorname{Hom}(C, \mathcal{P}, Y)=0$. 

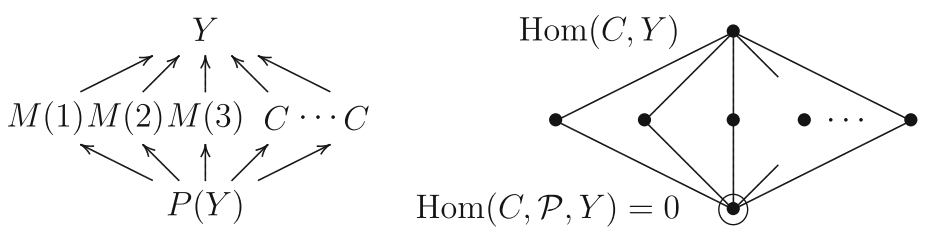

Example 18 where the modules in ${ }^{C}[\rightarrow Y\rangle^{1}$ form a family of modules with varying dimension vectors. Again, we take the 3 -subspace quiver as considered in example 9. Now let $C=P(a)$ and $Y=\tau Q(a)$ the maximal indecomposable module.

Since $C$ is the projective module $P(a)$, the right minimal right $C$-determined maps $f: X \rightarrow Y$ are the inclusions maps of submodules $X$ of $Y$ such that the socle of $Y / X$ is a direct sum of copies of $S(a)$. The indecomposable submodules of $Y$ are $P\left(b_{1}\right), P\left(b_{2}\right), P\left(b_{3}\right)$ as well as submodules isomorphic to $S(a)$. In order that the socle of $Y / X$ is a direct sum of copies of $S(a)$, either $X=Y$ (and $Y / X=0$ ), or $X=0$ (and $Y / X=Y$ ), or $X$ has to be indecomposable. If $X$ is an indecomposable proper submodule of $Y$, then the socle of $Y / X$ is either isomorphic to $S(0)$ or else $X$ is contained in one of the modules $P\left(b_{1}\right), P\left(b_{2}\right), P\left(b_{3}\right)$. It follows that the 1 parameter family in the middle of ${ }^{C}[\rightarrow Y\rangle$ consists of the indecomposable proper submodules $X$ of $Y$ such that the socle of $Y / X$ is isomorphic to $S(a)$, thus either $X$ is one of $P\left(b_{1}\right), P\left(b_{2}\right), P\left(b_{3}\right)$, or else $X$ is a simple submodule of $Y$ not contained in $P\left(b_{1}\right), P\left(b_{2}\right), P\left(b_{3}\right)$.

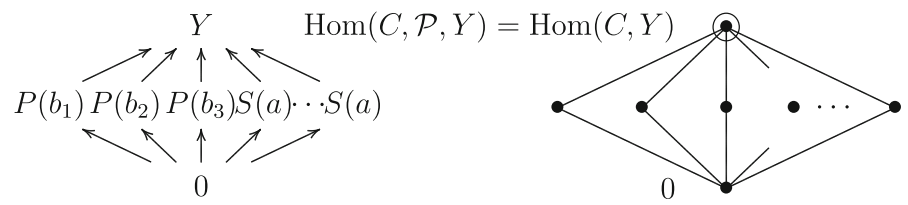

Example 19 Another example where the modules in ${ }^{C}[\rightarrow Y\rangle^{1}$ form a family of modules with varying dimension vectors. Take the one-point extension $\Lambda$ of the Kronecker algebra using a regular module $R$ of length 2 , say $R=R_{\infty}$

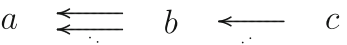

The vertex $c$ is the extension vertex and $\operatorname{rad} P(c)=R_{\infty}$. The regular Kronecker modules of length 2 different from $R_{\infty}$ will be denoted by $R_{\lambda}$ with $\lambda \in k$.

Let $K=S(a)$ and $C=\tau^{-} K$, this is the indecomposable Kronecker-module $P_{2}$, its dimension vector (as a $\Lambda$-module) is $(3,2,0)$. Let $Y=P(c) / S(a)$, its dimension vector is $(0,1,1)$. We have $\operatorname{dim} \operatorname{Hom}(C, Y)=2$. Since $\operatorname{End}(C)=k$, the submodule lattice $\mathcal{S} \operatorname{Hom}(C, Y)$ is just the projective line. Under $\eta_{S Y}$ we obtain a $\mathbb{P}_{1}$-family of right minimal maps ending in $Y$, namely those with the following short exact sequences:

$$
\begin{aligned}
& K \longrightarrow P(c) \stackrel{p}{\longrightarrow} Y \\
& K \longrightarrow R_{\lambda} \stackrel{f_{\lambda}^{\prime}}{\longrightarrow} S(b) \quad \text { for } \lambda \in k
\end{aligned}
$$

Here is, on the left, ${ }^{C}[\rightarrow Y\rangle$, and, on the right, $\mathcal{S} \operatorname{Hom}(C, Y)$ 

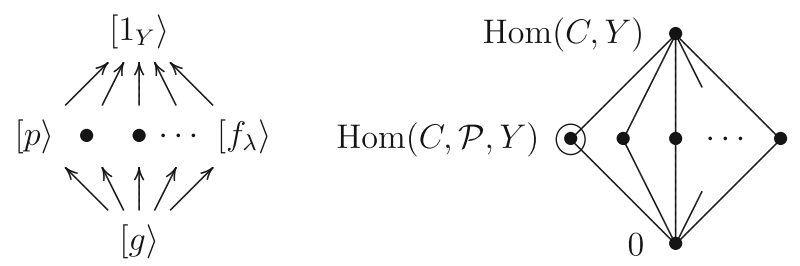

The 0-subspace of $\operatorname{Hom}(C, Y)$ corresponds to a map $g: P(b) \rightarrow Y$ with image $S(b)$, namely to the projective presentation of $S(b)$

$$
K^{2} \longrightarrow P(b) \stackrel{g^{\prime}}{\longrightarrow} S(b) .
$$

It should be noted that the short exact sequence

$$
K \longrightarrow R_{\infty} \stackrel{f_{\infty}^{\prime}}{\longrightarrow} S(b)
$$

yields the map $R_{\infty} \stackrel{f_{\infty}^{\prime}}{\longrightarrow} S(b) \subset Y$ which we denote by $f_{\infty}$ and which is not $C$ determined. Namely, there is the following commutative diagram

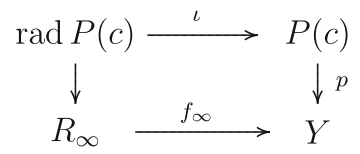

with inclusion map $\iota$. Since $p$ does not factor through $f_{\infty}$, we see that $p$ almost factors through $f_{\infty}$, thus the theory asserts that $P(c)$ has to belong to any determiner of $f_{\infty}$ and therefore $f_{\infty}$ cannot belong to ${ }^{C}[\rightarrow Y\rangle$.

As we have noted, $P(c)$ has to belong to any determiner of $f_{\infty}$. Let us add $P(c)$ to $C$ and consider the Auslander bijection for $C \oplus P(c)$ and $Y$. We have $\operatorname{dim} \operatorname{Hom}(C \oplus$ $P(c), Y)=3$. Note that the endomorphism ring of $C \oplus P(c)$ is hereditary of type $\mathbb{A}_{2}$ and the submodule structure of $\operatorname{Hom}(C \oplus P(c), Y)$ looks as follows:

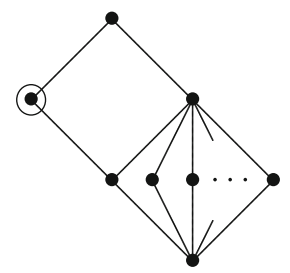

For the proof, we only have to verify that a non-trivial map $C \rightarrow P(c)$ does not annihilate the module $\operatorname{Hom}(C \oplus P(c), Y)$. The encircled vertex is the submodule $\operatorname{Hom}(C \oplus P(c), \mathcal{P}, Y)$.

The corresponding diagram in ${ }^{C \oplus P(c)}[\rightarrow Y\rangle$ looks as follows; here, we write its elements as short exact sequences ending in a submodule of $Y$ : 

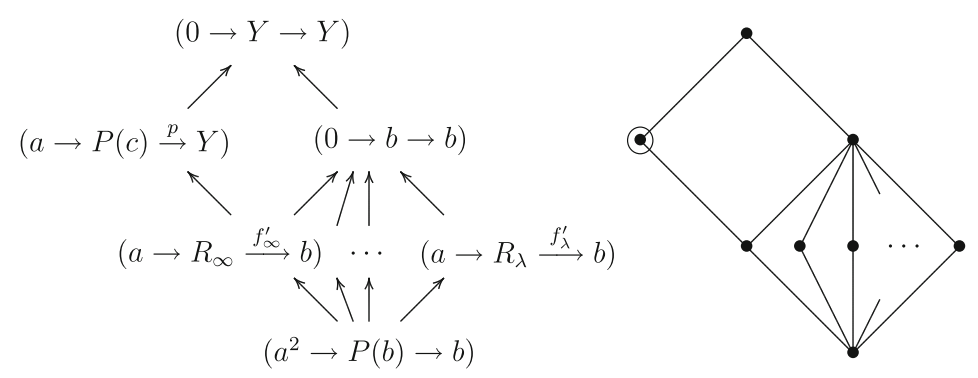

We may label the lines of the Hasse diagram of $\mathcal{S} \operatorname{Hom}(C \oplus P(c), Y)$ by the corresponding type, thus either by $C$ or by $P(c)$

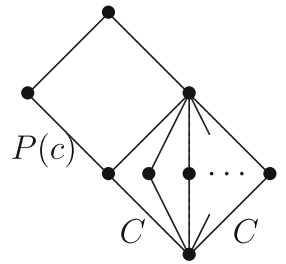

We should add that conversely, we can recover $\mathcal{S} \operatorname{Hom}(C, Y)$ from $\mathcal{S} \operatorname{Hom}(C \oplus$ $P(c), Y)$ by deleting the shaded part:

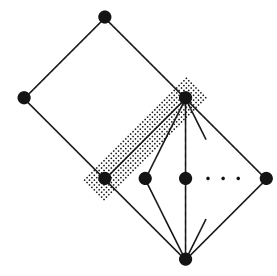

\section{Special cases}

\section{The module $C$ being a generator}

The special case $C=\Lambda$ has been discussed already at the end of Sect. 4 . In this case, $\mathcal{S} \operatorname{Hom}(C, Y)$ is just the lattice of all submodules of $Y$.

Proposition 16.1 Let $C$ be a generator. Then $f: X \rightarrow Y$ is right $C$-determined if and only if the intrinsic kernel of $f$ is in add $\tau C$.

Proof According to Theorem 3.4, the map $f$ is right $C$-determined if and only if $C(f) \in$ add $C$. Thus, assume that $C(f) \in$ add $C$. Let $K$ be an indecomposable direct summand of the intrinsic kernel of $f$. By definition of $C(f)$, we know that $\tau^{-} K$ belongs to add $C(f)$, and $K$ is not injective. Thus $K=\tau \tau^{-} K$ belongs to add $\tau C(f) \subseteq$ add $C$. This shows that the intrinsic kernel of $f$ belongs to add $\tau C$.

Conversely, assume that the intrinsic kernel belongs to add $\tau C$, in particular any indecomposable direct summand of the intrinsic kernel $K$ belongs to add $\tau C$, thus $\tau^{-} K$ belongs to add $\tau^{-} \tau C \subseteq$ add $C$. Also, since $C$ is a generator, any indecomposable 
projective module belongs to add $C$. As a consequence, $C(f)$ belongs to add $C$. This completes the proof.

Everyone admits that the concept of being determined is not very intuitive, however in the special case when $C$ is a generator (and this is often the only important case), one knows: The maps in ${ }^{C}[\rightarrow Y\rangle$ can be described by the exact sequences

$$
0 \rightarrow K \rightarrow X \stackrel{e}{\rightarrow} Y^{\prime} \rightarrow 0
$$

where $K$ is in add $\tau C$ and $Y^{\prime}$ is a submodule of $Y$ say with inclusion map $m: Y^{\prime} \rightarrow Y$, the map in ${ }^{C}[\rightarrow Y\rangle$ in question is the composition $m e$. To repeat: If $C$ is a generator, a right minimal is right $C$-determined if and only if its kernel belongs to add $\tau C$.

This means: for $C$ a generator, the set ${ }^{C}[\rightarrow Y\rangle$ can be visualized very well. Unfortunately, this seems to be difficult in general, but the notion of right determination just allows to have the prolific bijection ${ }^{C}[\rightarrow Y\rangle \leftrightarrow \mathcal{S} \operatorname{Hom}(C, Y)$.

Proposition 16.2 Let $C$ be a generator and $u: Y^{\prime} \rightarrow Y$ a monomorphism. Then $f \mapsto u f$ defines an embedding $u_{*}:{ }^{C}\left[\rightarrow Y^{\prime}\right\rangle \rightarrow{ }^{C}[\rightarrow Y\rangle$. The image of this embedding is an ideal of the lattice ${ }^{C}[\rightarrow Y\rangle$ and the following diagram commutes:

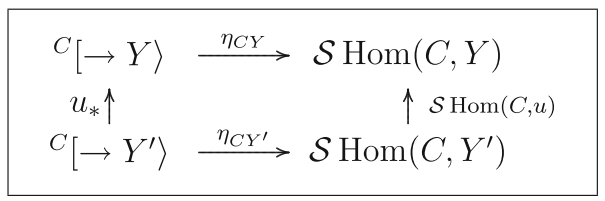

Proof Let $f: X \rightarrow Y^{\prime}$ be right $C$-determined. Since the intrinsic kernel of $f$ and of $u f$ are the same, also $u f$ is right $C$-determined by 14.1. If $f \preceq f^{\prime}$ are maps ending in $Y^{\prime}$, then there is a map $h$ such that $f=f^{\prime} h$, therefore $u f=u f^{\prime} h$, thus $u f \preceq u f^{\prime}$. This shows that $f \mapsto u f$ yields a map $u_{*}:{ }^{C}\left[\rightarrow Y^{\prime}\right\rangle \rightarrow^{C}[\rightarrow Y\rangle$. In order to see that $u_{*}$ is an embedding, let us assume that $f, f^{\prime}$ are maps ending in $Y$ such that $u f \preceq u f^{\prime}$. Then there is $h$ with $u f=u f^{\prime} h$, but this implies that $f=f^{\prime} h$ (since $u$ is a monomorphism), and therefore $f \preceq f^{\prime}$. In order to see that the image of $u_{*}$ is an ideal, let $f$ be right $C$-determined and ending in $Y^{\prime}$ and $g$ right $C$-determined ending in $Y$ such that $g \preceq u_{*}(f)$. We want to show that $g$ is in the image of $u_{*}$. But $g \preceq u f$ means that there is $h$ with $g=u f h$, thus $g=u_{*}(f h)$.

It remains to show that the diagram commutes. Let $f: X \rightarrow Y^{\prime}$ be right $C$ determined. Then we have $\eta_{C Y^{\prime}}(f)=f \operatorname{Hom}(C, X)$. The map $\operatorname{Hom}(C, u)$ send any $\phi \in \operatorname{Hom}\left(C, Y^{\prime}\right)$ to $\operatorname{Hom}(C, u)(\phi)=u \phi$, thus $\mathcal{S} \operatorname{Hom}(C, u)$ sends $f \operatorname{Hom}(C, X)$ to $u f \operatorname{Hom}(C, X)$. On the other hand, we also have $\eta_{C Y}\left(u_{*}(f)\right)=\eta_{C Y}(u f)=$ uf $\operatorname{Hom}(C, X)$.

If $u: Y^{\prime} \rightarrow Y$ is a monomorphism, but $C$ is not a generator, then given $[f\rangle \in$ ${ }^{C}\left[\rightarrow Y^{\prime}\right\rangle$, the right equivalence class of the composition $u f$ usually will not belong to ${ }^{C}[\rightarrow Y\rangle$.

Example 20 Take the quiver of type $\mathbb{A}_{2}$ as considered in example 1 and take $C=$ $Y=S(2)$ and $Y^{\prime}=0$ with inclusion map $u: 0 \rightarrow S(2)$. The zero map $0 \rightarrow 0$ yields an element of ${ }^{C}\left[\rightarrow Y^{\prime}\right\rangle$, but $u$ is not right $C$-determined, since $P(2)$ almost factors through $u$, so that $P(2)$ has to be a direct summand of $C(u)$ and therefore of any right determiner of $u$. 


\section{The case of $[\rightarrow Y\rangle$ being of finite height}

Here we consider modules $Y$ such that there are only finitely many indecomposable modules $M_{i}$ with $\operatorname{Hom}\left(M_{i}, Y\right) \neq 0$. Let $M$ be the direct sum of these modules and consider the Auslander bijection

$$
{ }^{M}[\rightarrow Y\rangle \leftrightarrow \mathcal{S} \operatorname{Hom}(M, Y),
$$

it maps $\bigoplus_{i, j} M_{i}^{n_{i}} \stackrel{\left(f_{i j}\right)}{\longrightarrow} Y$ to the $\Gamma(M)$-submodule of $\operatorname{Hom}(M, Y)$ generated by the maps $f_{i j}$ (considered as maps $M \rightarrow M_{i} \rightarrow Y$ where the map $M \rightarrow M_{i}$ is the canonical projection onto the direct summand).

Proposition 17.1 Let $Y$ be a module.

(a) If $[\rightarrow Y\rangle$ is of height $h$, then the number of isomorphism classes of indecomposable modules $M_{i}$ with $\operatorname{Hom}\left(M_{i}, Y\right) \neq 0$ is at most $h$.

(b) If $M_{1}, \ldots, M_{t}$ are all the indecomposable modules $M_{i}$ with $\operatorname{Hom}\left(M_{i}, Y\right) \neq 0$, one from each isomorphism class, and $M=\bigoplus_{i}^{t} M_{i}$, then $[\rightarrow Y\rangle={ }^{M}[\rightarrow Y\rangle$ and any module $C$ with $[\rightarrow Y\rangle={ }^{C}[\rightarrow Y\rangle$ satisfies $M \in$ add $C$.

Proof Let $M_{1}, \ldots, M_{t}$ be pairwise non-isomorphic indecomposable modules with $\operatorname{Hom}\left(M_{i}, Y\right) \neq 0$, and let $M=\bigoplus_{i}^{t} M_{i}$. Let $\Gamma(M)=\operatorname{End}(M)^{\mathrm{op}}$. Then the indecomposable projective $\Gamma(M)$-modules $P(i)=\operatorname{Hom}\left(M, M_{i}\right)$ are pairwise non-isomorphic, and $\operatorname{Hom}_{\Gamma(M)}(P(i), \operatorname{Hom}(M, Y)) \neq 0$, thus the $\Gamma(M)$-module $\operatorname{Hom}(M, Y)$ has length at least $t$. Now, according to the Auslander bijection, the poset $\mathcal{S} M$ is isomorphic to the subposet ${ }^{M}[\rightarrow Y\rangle$ of $[\rightarrow Y\rangle$. The length of $\mathcal{S} M$ is at least $t$, the length of $[\rightarrow Y\rangle$ is $h$. This shows that $t \leq h$. This shows (a).

In order to show (b), we recall from Proposition 3.9 that given a map $f$ ending in $Y$, any indecomposable direct summand $C_{0}$ of $C(f)$ satisfies $\operatorname{Hom}\left(C_{0}, Y\right) \neq 0$, thus $C_{0}$ is in add $M$ and therefore $C(f) \in$ add $M$. Thus any map $f: X \rightarrow Y$ is right $M$-determined and therefore $[\rightarrow Y\rangle={ }^{M}[\rightarrow Y\rangle$.

On the other hand, let us assume that $[\rightarrow Y\rangle={ }^{C}[\rightarrow Y\rangle$ for some module $C$. Using again 3.9, we can assume that any indecomposable direct summand $C_{i}$ of $C$ satisfies $\operatorname{Hom}\left(C_{i}, Y\right) \neq 0$. Thus we can assume that $C$ is a direct summand of $M$. But if $C$ is a proper direct summand of $M$, say $C=\bigoplus_{i=1}^{t^{\prime}} M_{i}$ with $t^{\prime}<t$, then

$$
\begin{aligned}
|[\rightarrow Y\rangle| & =\left|{ }^{C}[\rightarrow Y\rangle\right|=|\operatorname{Hom}(C, Y)| \\
& =\sum_{i=1}^{t^{\prime}}\left|\operatorname{Hom}\left(M_{i}, M\right)\right|<\sum_{i=1}^{t}\left|\operatorname{Hom}\left(M_{i}, M\right)\right| \\
& =|\operatorname{Hom}(M, Y)|=\left.\right|^{M}[\rightarrow Y\rangle|\leq|[\rightarrow Y\rangle \mid
\end{aligned}
$$

a contradiction. This shows that $C=M$.

Remark As we see, the indecomposable modules $M_{i}$ which occur as direct summands of a minimal module $C$ with $[\rightarrow Y\rangle={ }^{C}[\rightarrow Y\rangle$ are modules with $\operatorname{Hom}\left(M_{i}, Y\right) \neq 0$. But this is not surprising, since the minimal right determiner $C(f)$ of any morphism $f$ has as indecomposable direct summands only modules $C_{i}$ with $\operatorname{Hom}\left(C_{i}, Y\right) \neq 0$, see 
3.9. Also the converse should be stressed, namely the following part of the assertion (b): any indecomposable module $M_{i}$ with $\operatorname{Hom}\left(M_{i}, Y\right) \neq 0$ is needed as a direct summand of $C$.

Corollary 17.2 Let $Y$ be a module. The following conditions are equivalent:

(i) There are only finitely many isomorphism classes of indecomposable modules $X$ with $\operatorname{Hom}(X, Y) \neq 0$.

(ii) The lattice $[\rightarrow Y\rangle$ has finite height.

(iii) There is a module $C$ with $[\rightarrow Y\rangle={ }^{C}[\rightarrow Y\rangle$.

Proof The implication (ii) $\Longrightarrow$ (i) has been shown in 17.1(a), the implication (i) $\Longrightarrow$ (iii) in 15.1(b). Any lattice of the form ${ }^{C}[\rightarrow Y\rangle$ is of finite height, thus obviously (iii) implies (ii).

If $S$ is simple and $[\rightarrow Q(S)\rangle$ is of finite height, then we deal just with the hammock corresponding to $S$, as considered in [40].

Example 21 Let $\Lambda$ be the 3-subspace quiver as considered in example 9 and let $Y=$ $Q(a)$. As before, we denote the indecomposable modules which are neither projective nor injective by $N(i)=\tau Q\left(b_{i}\right)$ with $1 \leq i \leq 3$ and $M=\tau Q(a)$. Here is the lattice $[\rightarrow Y\rangle$

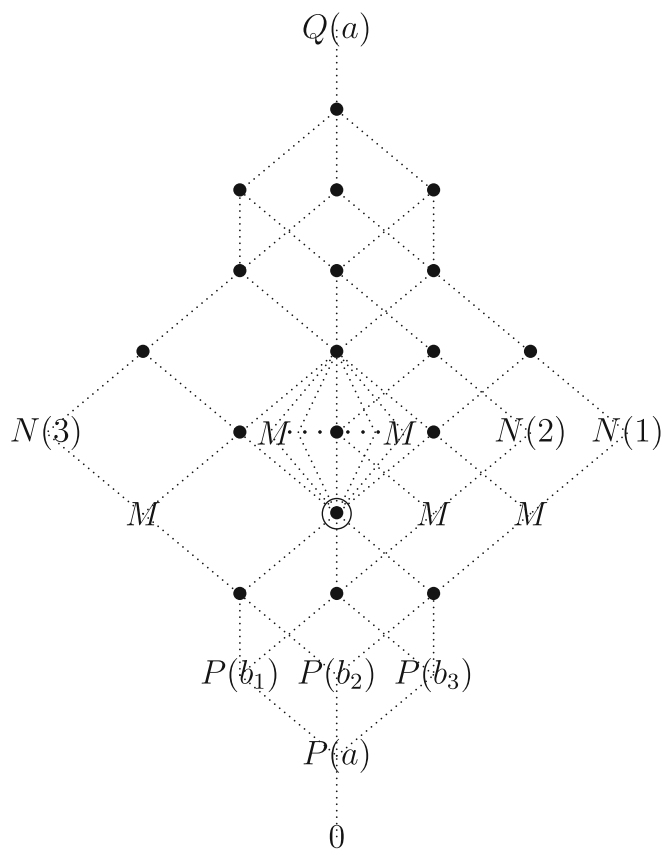

Let $A$ be the direct sum of all indecomposable $\Lambda$-modules, one from each isomorphism class. Then the $\Gamma(A)$-module $\operatorname{Hom}(A, Y)$ is of length 10 with 9 different composition factors, they correspond to the indecomposables

$$
P(a) ; P\left(b_{1}\right), P\left(b_{2}\right), P\left(b_{3}\right) ; M ; N(1), N(2), N(3) ; Q(a),
$$


and the composition factor corresponding to $M$ occurs with multiplicity 2 in $\operatorname{Hom}(A, Y)$.

In the picture above, only join irreducible elements (as well as the zero element) have been labeled. Note that in the middle layer of the non-distributive interval of length 2, all but three elements are join irreducible, and have the label $M$; here we deal with the various epimorphisms $M \rightarrow Q(0)$. Altogether there are 18 non-zero elements which are not join-irreducible.

Note that the picture is obtained from the free modular lattice in 3 generators as presented by Dedekind [11] in 1900 by inserting in the non-distributive interval of length 2 further diagonals (one may call it the free $k$-modular lattice in 3 generators).

There is an obvious action of the symmetric group $S_{3}$ of order 6 on the 3 -subspace quiver, and thus also on the lattice $[\rightarrow Y\rangle$. Six vertices of $[\rightarrow Y\rangle$ are invariant under this action, five of them correspond to important maps ending in $Y$ :

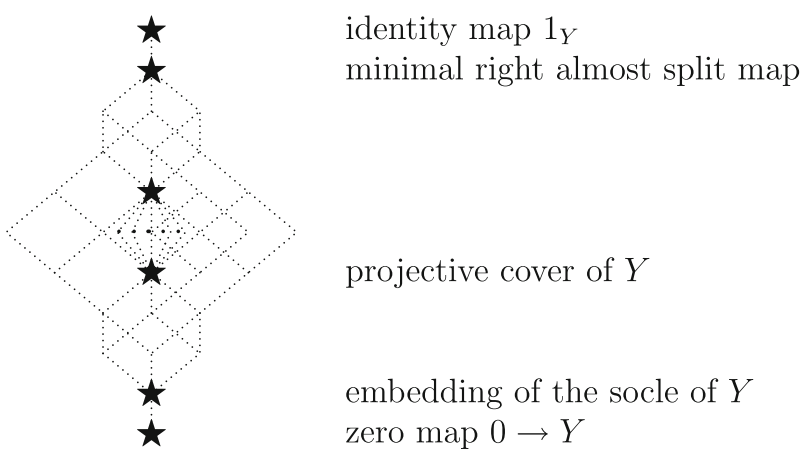

the remaining one is the universal map from add $M$ to $Y$.

Instead of looking at representatives $f$ in right equivalence classes, we may also draw the attention on the right equivalence classes $[f\rangle$ themselves. For example, $\left[1_{Y}\right\rangle$ is the class of all split epimorphisms ending in $Y$. If $g: X \rightarrow Y$ is minimal right almost split, then $[g\rangle$ is the class of all right almost split maps ending in $Y$ and finally, $[0 \rightarrow Y\rangle$ is the class all zero maps ending in $Y$.

Remark An attempt to deal in a similar way with the $n$-subspace quivers for $n \geq 4$ was given by Gelfand and Ponomarev in a series of papers [16-19], see also [13] and [32]. In order to get information about the free modular lattice in $n$ generators, they described part of the lattice $[\rightarrow Q(a)\rangle$ where $\Delta$ is the $n$-subspace quiver and $Q(a)$ is the indecomposable injective $k \Delta$-module with $a$ the sink of $\Delta$. Of course, for $n \geq 4$, this lattice [ $\rightarrow Q(a)\rangle$ is of infinite height! It may be worthwhile to look for an interpretation of the results of Gelfand-Ponomarev in terms of the Auslander bijections.

\section{Some serial modules $\operatorname{Hom}(C, Y)$}

The Auslander bijections are defined for any pair of $\Lambda$-modules $C, Y$ and one of the posets involved is $\mathcal{S} \operatorname{Hom}(C, Y)$. Assume that $J$ is an ideal of $\Lambda$ which annihilates 
both modules $C, Y$ so that we may consider $C$ and $Y$ as $\Lambda^{\prime}$-modules, with $\Lambda^{\prime}=\Lambda / J$. On the one hand, we have $\operatorname{Hom}_{\Lambda}(C, Y)=\operatorname{Hom}_{\Lambda^{\prime}}(C, Y)$. On the other hand, we have to distinguish the set ${ }^{C}[\rightarrow Y\rangle_{\Lambda^{\prime}}$ of right equivalence classes of right $C$-determined $\Lambda^{\prime}$-modules ending in $Y$ from ${ }^{C}[\rightarrow Y\rangle_{\Lambda}={ }^{C}[\rightarrow Y\rangle$. Using the Auslander bijections for $\Lambda$ as well as for $\Lambda^{\prime}$, it is clear that the posets ${ }^{C}[\rightarrow Y\rangle_{\Lambda^{\prime}}$ and ${ }^{C}[\rightarrow Y\rangle_{\Lambda}$ are isomorphic, however the modules present in ${ }^{C}[\rightarrow Y\rangle_{\Lambda^{\prime}}$ usually will be completely different from those present in ${ }^{C}[\rightarrow Y\rangle_{\Lambda}$. The following examples will show such deviations.

Let $\Lambda$ be a local uniserial ring. When dealing with a local uniserial ring, the indecomposable module of length $n$ will be denoted just be $n$. Also, if $C$ is a module, we will write $\operatorname{rad}^{t}(C, C)$ instead of $(\operatorname{rad} \operatorname{End}(C))^{t}$.

Example 22 Let $C=Y=4$. First, let $\Lambda$ be of length at least 8 , so that $\operatorname{Hom}(4, \mathcal{P}$, 4) $=0$.
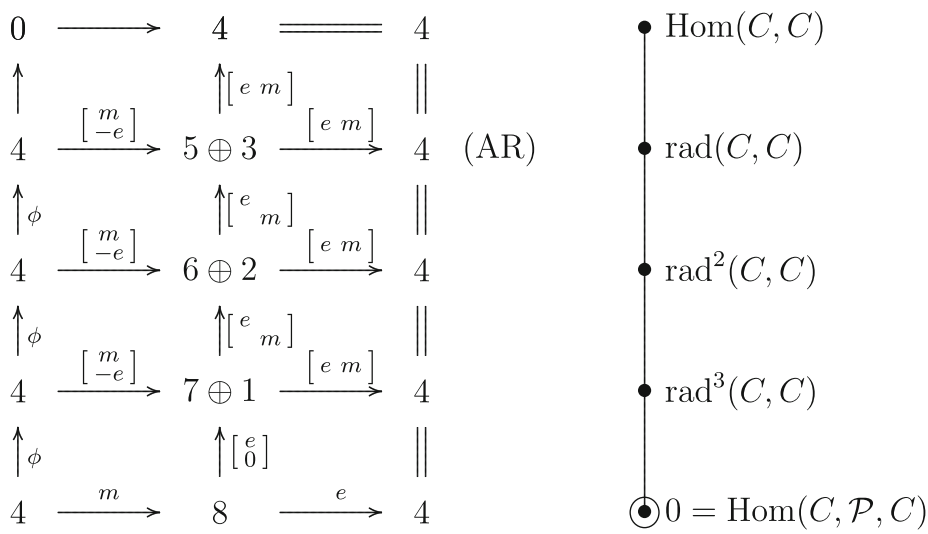

here, we have denoted by $m$ the canonical inclusion maps, by $e$ the canonical projections and $\phi$ is a radical generator $\operatorname{End}(C)$. The Auslander-Reiten sequence is marked as (AR).

Second, let $\Lambda$ be of length 6 , so that $\operatorname{Hom}(4, \mathcal{P}, 4)=\operatorname{rad}^{2}(C, C)$.
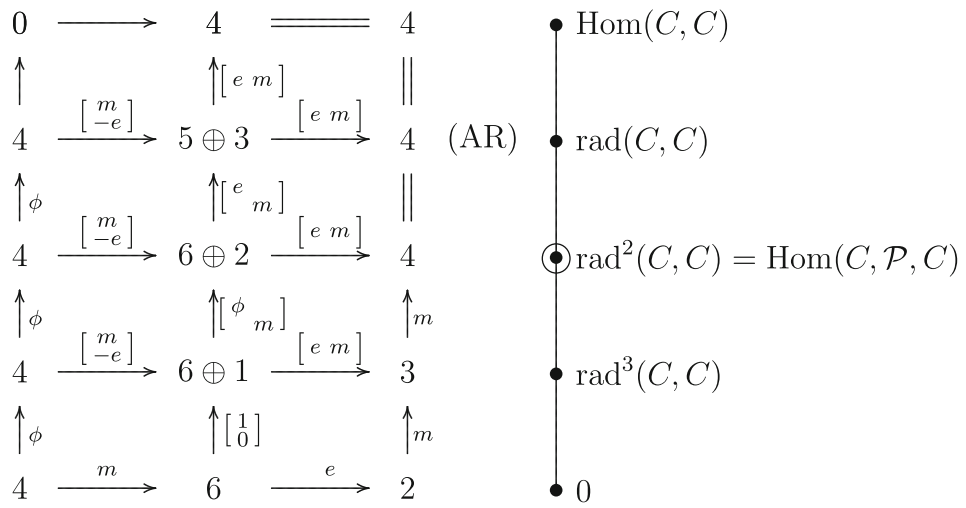

again, $m$ stands for a canonical inclusion map, $e$ for a canonical projection map and $\phi$ for a radical generator of the endomorphism ring of a uniserial module. 
Finally, let $\Lambda$ be of length 4 , thus $C$ is projective and therefore $\operatorname{Hom}(C, \mathcal{P}, C)=$ $\operatorname{Hom}(C, C)$. Since $C$ is projective, all the right minimal, right $C$-determined maps are inclusion maps:

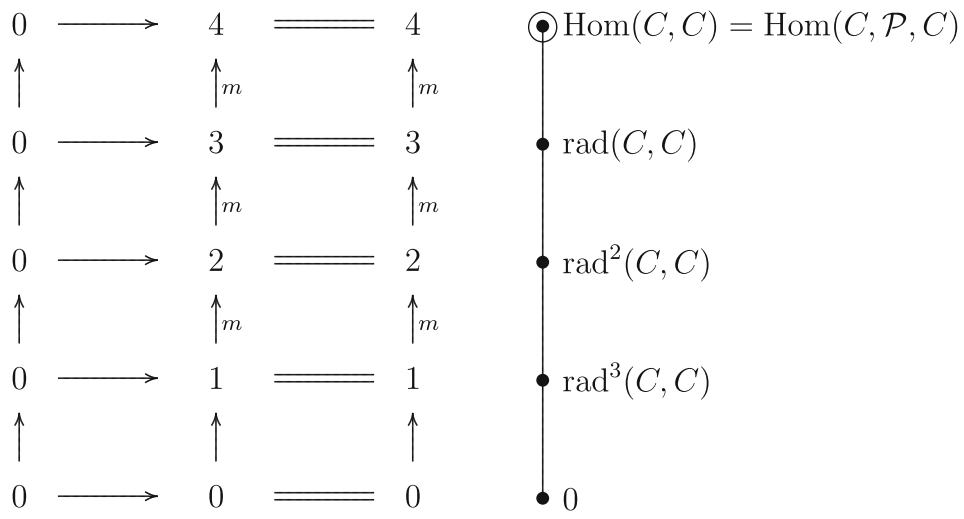

Example 23 Let $C=1 \oplus 2$ and $Y=3$. The ring $\Gamma(C)=\operatorname{End}(C)^{\text {op }}$ is the Nakayama algebra with Kupisch series 2, 3. The $\Gamma(C)$-module $\operatorname{Hom}(C, Y)$ is the indecomposable projective module of length 3 . If we work over a uniserial ring $\Lambda$ of length at least 5 , so that $\operatorname{Hom}(C, \mathcal{P}, Y)=0$, then the situation is as follows:
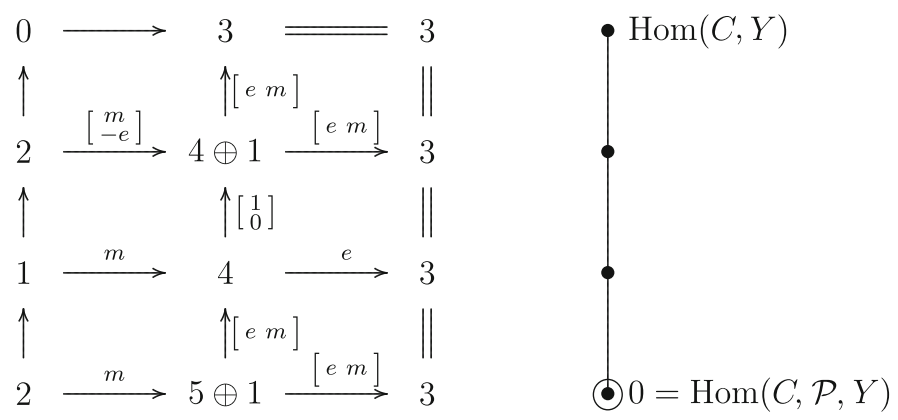

Next, assume that $\Lambda$ is of length 4 .
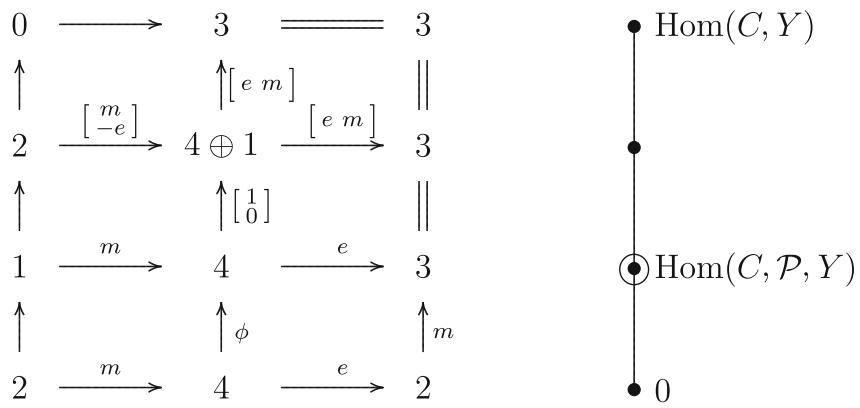
Finally, we consider the case where $\Lambda$ is of length 3 .
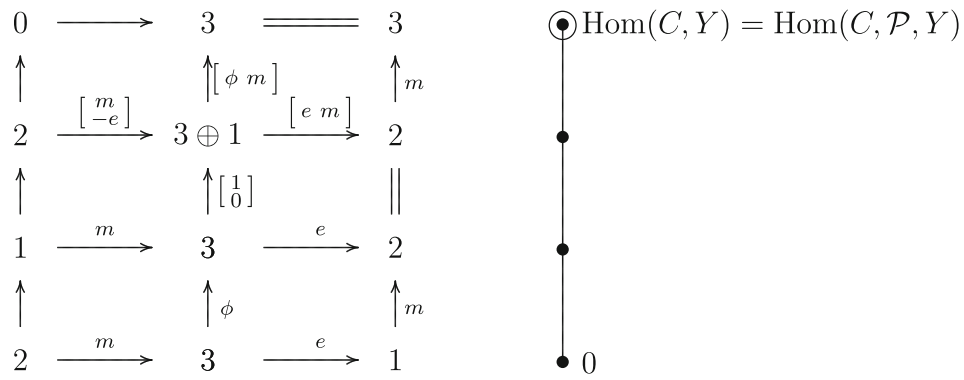

\section{Final remarks}

\subsection{Duality}

For all the results presented here, there is a dual version which has the same importance. Note that we deal with an artin algebra $\Lambda$ and consider finitely generated modules, thus there is a duality functor. Namely, by assumption, $\Lambda$ is a module-finite $k$-algebra, where $k$ is a commutative artinian ring. A minimal injective cogenerator $Q$ in $\bmod k$ yields the duality functor $\operatorname{Hom}_{k}(-, Q)$.

We did not attempt to formulate the dual definitions and statements, but the reader is advised to do so. To start with, we need the notion of left equivalence in order to introduce $\langle Y \rightarrow$ ] as the set of left equivalence classes of maps starting in $Y$ (or of left minimal maps). Then we need the notion of left $C$-determination in order to define $\langle Y \rightarrow]^{C}$ as the set of the left equivalence classes maps starting in $Y$ which are left $C$-determined.

\subsection{Proofs of Auslander's two main theorems}

Both results are presented in detail in the Philadelphia notes [2], see also [3]. There is a different treatment in the book [5] of Auslander-Reiten-Smalø: Auslander's Second Theorem is presented in Theorem XI.3.9., for the First Theorem, see Theorem XI.2.10 and Proposition XI.2.4 (this actually improves the assertion given in the Philadelphia Notes). A concise proof of the First Theorem can also be found in [38]. As one of the main ingredients for the proof of the Second Theorem, one may use Auslander's defect formula. For a direct approach to the defect formula, we recommend the paper [24] by Krause.

\subsection{The universal character of the Auslander bijections}

The Auslander bijections are Auslander's approach to describe say the module category for an artin algebra completely: not just to provide some invariants. The importance of using invariants usually relies on the fact that they will allow to distinguish certain 
objects, but they may not say much about other ones-the most effective invariants are often those which attach to objects just one of the values 1 or 0 (thus "yes" or "no"). Of course, the use of such an invariant is restricted to some specific problem. Now Auslander's approach is an attempt to describe a module category completely, thus we may ask whether it does not have to be tautological: if we don't forget some of the structure, we will not see the remaining structure in more detail. Indeed, the Auslander bijections focus on parts of the category, namely the sets ${ }^{C}[\rightarrow Y\rangle$, but the decisive feature is the possibility to change the focus by enlarging $C$ (adding direct summands). The universality of this approach is due to the fact that the category is covered completely by such subsets.

\subsection{The irritation of the wording "morphisms determined by modules"}

Let us insert a short discussion of our hesitation to say that a morphism $f$ is right determined by a module $C$. One possible interpretation is to assert that even if the morphism $f$ itself is not determined by $C$, there is a certain factorization property of $f$ which is determined by $C$. But there is another way out:

When Auslander asserts that every morphism in $\bmod \Lambda$ is right determined by a $\Lambda$-module, one expects a classification of the (right minimal) morphisms in $\bmod \Lambda$ using as invariants just $\Lambda$-modules. One even may strengthen Auslander's assertion by saying every morphism in $\bmod \Lambda$ is right determined by the isomorphism class of a multiplicity-free $\Lambda$-module. Clearly, such a formulation is irritating, since the set of isomorphism classes of multiplicity-free modules may outnumber the set of right equivalence classes of right minimal morphisms by far: Consider just the special case of a representation finite artin algebra of uncountable cardinality, then there are only finitely many isomorphism classes of multiplicity-free modules, but usually uncountably many right equivalence classes of right minimal morphisms. So how should it be possible that finitely many modules $C$ determine uncountably many morphisms $\alpha$ ? The solution is rather simple: it is not just the module $C$ which is needed to recover a morphism $\alpha: X \rightarrow Y$ but one actually needs a submodule of $\operatorname{Hom}(C, Y)$, with $\operatorname{Hom}(C, Y)$ being considered as an $\operatorname{End}(C)^{\text {op }}$-module. In the setting where $\Lambda$ is representation-finite and uncountable, one should be aware that usually the modules $\operatorname{Hom}(C, Y)$ will have uncountably many submodules, thus we are no longer in trouble. So if we assert that the morphism $\alpha: X \rightarrow Y$ is determined by the module $C$, then one should keep in mind that $C$ is only part of the data which are required to recover $\alpha$; in addition to $C$ one will need a submodule of $\operatorname{Hom}(C, Y)$.

\subsection{Modules versus morphisms, again}

The following feature seems to be of interest: The concept of the determination of morphisms by modules concerns the category of maps with fixed target $Y$, namely one wants to decide whether two elements in $[\rightarrow Y\rangle$ are comparable. The theory asserts that there is a test set of modules, namely the indecomposable direct summands of 
$C(f)$. For the testing procedure, they are just modules, but any such object $L$ comes equipped with a non-zero (thus right minimal) map $L \rightarrow Y$.

\subsection{Logic and category theory}

Let us stress that the setting of the Auslander bijections is well-accepted both in mathematical logic and in category theory. The lattice of $\Lambda$-pointed modules, as studied in model theory (see for example [28]) is precisely the lattice $\langle\Lambda \rightarrow$ ]. In this way, all the results concerning pp-formulae and pp-definable subgroups concern the Auslander setting.

In the terminology of abstract category theory, we deal with a comma category, namely the category of objects over $Y$ : its objects are the maps $X \rightarrow Y$, a map from $f: X \rightarrow Y$ to $f^{\prime}: X^{\prime} \rightarrow Y$ being given by a map $h: X \rightarrow X^{\prime}$ such that $f=f^{\prime} h$.

Of course, the use of the representable functors $\operatorname{Hom}(X,-)$ and $\operatorname{Hom}(-, Y)$ is standard in representation theory. To provide here all relevant references would overload our presentation due to the abundance of such papers. So we restrict to mention only few names: of course Auslander himself, but also Gabriel [14] as well as GelfandPonomarev.

\subsection{Generalisations}

In this survey we have restricted the attention on the module category of an artin algebra $\Lambda$, or, what is the same, a length $k$-category $\mathcal{C}$, where $k$ is a commutative artinian ring, such that $\mathcal{C}$ is Hom- and Ext-finite, has only finitely many simple objects and all objects have bounded Loewy length. Auslander's investigations were devoted to larger classes of rings, and many of our considerations should be of interest in a broader context.

Krause [25] has considered the general setting of dualising varieties in the sense of Auslander and Reiten [4] and has obtained the precise analogues of the Auslander Theorems which form the basis of our survey. In particular, he was looking also at triangulated categories and showed the relationship to the existence of Serre duality. The interested reader may try to work out in which way our presentation has to be modified in this context.

Acknowledgments Some of the material presented here has been exhibited in 2012 in lectures at SJTU, Shanghai, at USTC, Hefei, and at the ICRA conference at Bielefeld, in 2013 in lectures at Woods Hole and at NEU, Boston. The author is grateful to the audience for questions and remarks. He also wants to thank Xiao-Wu Chen, Lutz Hille, Henning Krause and Idun Reiten for helpful comments.

Open Access This article is distributed under the terms of the Creative Commons Attribution License which permits any use, distribution, and reproduction in any medium, provided the original author(s) and the source are credited.

\section{Appendix: Modular lattices}

Let $L$ be a modular lattice of height $h$. The set of elements in $L$ of height $i$ will be denoted by $L_{i}$ or also $L^{h-i}$. Here are some typical modular lattices: 
The chain $\mathbb{I}(h)$ of height $h$, this is the poset of the integers $0,1 \ldots, h$ with the usual order relation. Note that $\mathbb{I}(h)_{i}$ consists of a single element, for $0 \leq i \leq h$. Here are the lattices $\mathbb{I}(i)$ with $0 \leq i \leq 4$.

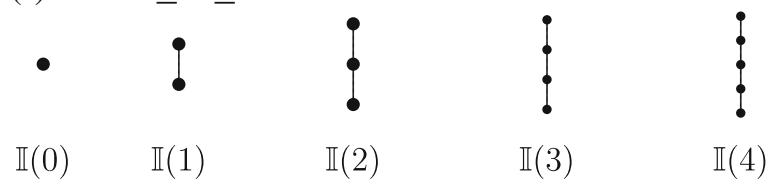

Let $k$ be a field. A projective geometry over $k$ is the lattice of all subspaces of a vector space over $k$. In accordance to the notation used throughout the paper, we denote the lattice of subspaces of the $d$-dimensional vector space $k^{d}$ by $\mathcal{S}\left(k^{d}\right)$ or also just by $\mathbb{G}(d)$ (this is called the projective geometry over $k$ of dimension $d-1$, it is a lattice of height $d$ ). Here are the lattices $\mathbb{G}(d)$ for $0 \leq d \leq 4$ :

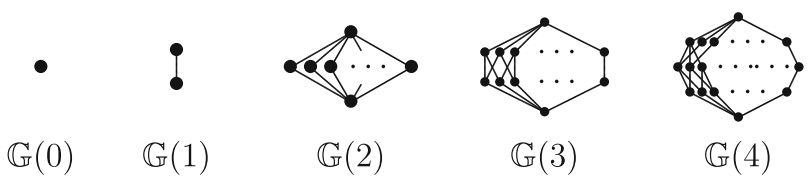

Of course, for $d \geq 2$, the number of elements of $\mathbb{G}(d)$ depends on the cardinality of $k$. If $k$ is a field with $q$ elements, the cardinality of $\mathbb{G}(2)_{1}=\mathbb{G}(2)^{1}$ is $q+1$ (here, $q$ is an arbitrary power of a prime number, thus if $\mathbb{G}(2)$ is finite, then $\left|\mathbb{G}(2)_{1}\right|=$ $3,4,5,6,8,9,10,12,14, \ldots)$.

The subset $\mathbb{G}(d)_{i}$ is the set of subspaces of dimension $i$ of a $k$-space of dimension $d$, it is often denoted by $\mathbb{G}(i, d-i)$ and called a Grassmannian; both sets $\mathbb{G}(d)_{1}$ and $\mathbb{G}(d)^{1}$ are also denoted by $\mathbb{P}^{d-1}$ and called the projective space of dimensiond -1 :

$$
\begin{array}{ll}
\mathbb{G}(d)_{d-1} & =\mathbb{G}(d)^{1}=\mathbb{P}^{d-1} \\
\mathbb{G}(d)_{i} & =\mathbb{G}(d)^{d-i}=\mathbb{G}(i, d-i) \\
\mathbb{G}(d)_{1} & =\mathbb{G}(d)^{d-1}=\mathbb{P}^{d-1} .
\end{array}
$$

\section{References}

1. Auslander, M., Reiten, I., Smalø, S.: Representation theory of Artin algebras. In: Cambridge Studies in Advanced Mathematics (1994)

2. Auslander, M.: Functors and morphisms determined by objects. In: Representation Theory of Algebras. Lecture Notes in Pure Appl. Math., vol. 37, pp. 1-244. Marcel Dekker, New York (1978). Also in: Selected Works of Maurice Auslander. Amer. Math. Soc. (1999)

3. Auslander, M.: Applications of morphisms determined by objects. In: Representation Theory of Algebras. Lecture Notes in Pure Appl. Math., vol. 37, pp. 245-327. Marcel Dekker, New York (1978). Also in: Selected Works of Maurice Auslander, Amer. Math. Soc. (1999)

4. Auslander, M., Reiten, I.: Stable equivalences of dualizing $R$-varieties. Adv. Math. 12, 306-366 (1974)

5. Auslander, M., Reiten, I., Smal $\varnothing$, S.: Representation Theory of Artin Algebras. Cambridge Studies in Advanced Mathematics, vol. 36. Cambridge University Press, Cambridge (1997)

6. Baez, J.: The n-category cafe: quivering with excitement. Blog May 4, 2012. http://golem.ph.utexas. edu/category/2012/05/quivering_with_excitement.html 
7. Bernstein, I.N., Gelfand, I.M., Ponomarev, V.A.: Coxeter functors and Gabriel's theorem. Russ. Math. Surv. 28, 17-32 (1973)

8. Bongartz, K., Huisgen-Zimmermann, B.: Varieties of uniserial representations IV. Kinship to geometric quotients. Trans. Am. Math. Soc. 353, 2091-2113 (2001)

9. Caldero, P., Chapoton, F.: Cluster algebras as Hall algebras of quiver representations. Comment. Math. Helv. 81, 595-616 (2006)

10. Crawley-Boevey, W.: On homomorphisms from a fixed representation to a general representation of a quiver. Trans. Am. Math. Soc. 348(5), 1909-1919 (1996)

11. Dedekind, R.: Über die von drei Moduln erzeugte Dualgruppe. Math. Ann. 53, 371-403 (1900)

12. Derksen, H., Huisgen-Zimmermann, B., Weymann, J.: Top stable degenerations of finite dimensional, representations II. (2013, to appear)

13. Dlab, V., Ringel, C.M.: Perfect elements in the free modular lattices. Math. Ann. 247, 95-100 (1980)

14. Gabriel, P.: Representations indecomposables. Sem. Bourbaki (1973-1974). LNM, vol. 431, pp. 143160. Springer, Berlin (1975)

15. Gelfand, I.M., Ponomarev, V.A.: Indecomposable representations of the Lorentz group. Russ. Math. Surv. 23, 1-58 (1968)

16. Gelfand, I.M., Ponomarev, V.A.: Free modular lattices and their representations. Russ. Math. Surv. 29, 1-56 (1974)

17. Gelfand, I.M., Ponomarev, V.A.: Lattices, representations and algebras connected with them I. Russ. Math. Surv. 31, 67-85 (1976)

18. Gelfand, I.M., Ponomarev, V.A.: Lattices, representations and algebras connected with them II. Russ. Math. Surv. 32, 91-114 (1977)

19. Gelfand, I.M., Ponomarev, V.A.: Representations of graphs. Perfect subrepresentations. Funkc. Anal. i Pril 13, 14-31. Funct. Anal. Appl. 14(1980), 177-190 (1980)

20. Grätzer, G.: Lattice Theory: Foundation. Birkhäuser, Basel (2011)

21. Hille, L.: Tilting line bundles and moduli of thin sincere representations of quivers. An. St. Univ. Ovidius Constantza 4, 76-82 (1996)

22. Huisgen-Zimmermann, B.: The geometry of uniserial representations of finite dimensional algebras I. J. Pure Appl. Algebra 127, 39-72 (1998)

23. Kraft, H.: Geometric methods in representation theory. In: Representations of Algebras, Lecture Notes in Math., vol. 944, pp 180-257. Springer, New York (1980)

24. Krause, H.: A short proof for Auslander's defect formula. Linear Algebra Appl. 365, 267-270 (2003)

25. Krause, H.: Morphisms determined by objects in triangulated categories. arXiv: 1110.5625

26. Le Bruyn, L.: Quiver Grassmannins can be anything. Blog, May 2, 2012. http://www. neverendingbooks.org/quiver-grassmannians-can-be-anything

27. MacLane, S.: Categories for the Working Mathematician. Springer, Berlin (1998)

28. Prest, M.: Purity, Spectra and Localisation, Encyclopedia of Mathematics and its Applications, vol. 121. Cambridge University Press, Cambridge (2009)

29. Reineke, M.: Every projective variety is a quiver Grassmannian. Algebras and Representation Theory (to appear). arXiv:1204.5730

30. Ringel, C.M.: Report on the Brauer-Thrall conjectures. In: Proceedings ICRA 2. LNM, vol. 831, pp. 104-136. Springer, Berlin (1980)

31. Ringel, C.M.: The rational invariants of tame quivers. Invent. Math. 58, 217-239 (1980)

32. Ringel, C.M.: Four papers on problems in lniear algebra. In: Representation Theory. Selected Papers by Gelfand et al. London Math Soc. Lecture Note Series, vol. 69. Cambridge University Press, Cambridge (1982)

33. Ringel, C.M.: Tame algebras and integral quadratic forms. In: Lecture Notes in Math., vol. 1099. Springer, New York (1984)

34. Ringel, C.M.: Hall polynomials for the representation-finite hereditary algebras. Adv. Math. 84, 137178 (1990)

35. Ringel, C.M.: The Gabriel-Roiter measure. Bull. Sci. Math. 129, 726-748 (2005)

36. Ringel, C.M.: Foundation of the representation theory of artin algebras, using the Gabriel-Roiter measure. In: de la Pena, Bautista (eds.) Trends in Representation Theory of Algebras and Related Topics. Contemporary Math. vol. 406, pp. 105-135. Amer. Math. Soc., New York (2006)

37. Ringel, C.M.: The first Brauer-Thrall conjecture. In: Models, Modules and Abelian Groups. In: Goldsmith, B., Göbel, R. (eds.) Memory of A. L. S. Corner, pp. 369-374. Walter de Gruyter, Berlin (2008) 
38. Ringel, C.M.: Morphisms determined by objects: the case of modules over artin algebras. Illinois J. (2013, to appear)

39. Ringel, C.M.: Quiver Grassmannians and Auslander varieties for wild algebras (in preparation)

40. Ringel, C.M., Vossieck, D.: Hammocks. Proc. London Math. Soc. 54(3), 216-246 (1987)

41. Roiter, A.V.: Unboundedness of the dimensions of the indecomposable representations of an algebra which has infinitely many indecomposable representations. Izv. Akad. Nauk SSSR Ser. Mat. 32, 12751282 (1968)

42. Schofield, A.: General representations of quivers. Proc. Lond. Math. Soc. 65(3) (1992) 